\title{
Taxonomic Revision of Trisetum Section Acrospelion (Poaceae: Pooideae: Aveninae) from Eurasia
}

Author(s): Patricia Barberá, Carlos Romero-Zarco, and Carlos Aedo

Source: Systematic Botany, 42(4):1-28.

Published By: The American Society of Plant Taxonomists

URL: http://www.bioone.org/doi/full/10.1600/036364417X696375

BioOne (www.bioone.org) is a nonprofit, online aggregation of core research in the biological, ecological, and environmental sciences. BioOne provides a sustainable online platform for over 170 journals and books published by nonprofit societies, associations, museums, institutions, and presses.

Your use of this PDF, the BioOne Web site, and all posted and associated content indicates your acceptance of BioOne's Terms of Use, available at www.bioone.org/page/terms_of_use.

Usage of BioOne content is strictly limited to personal, educational, and non-commercial use. Commercial inquiries or rights and permissions requests should be directed to the individual publisher as copyright holder. 


\title{
Taxonomic Revision of Trisetum section Acrospelion (Poaceae: Pooideae: Aveninae) from Eurasia
}

\author{
Patricia Barberá, ${ }^{1,3}$ Carlos Romero-Zarco, ${ }^{2}$ and Carlos Aedo ${ }^{1}$ \\ ${ }^{1}$ Real Jardín Botánico, Consejo Superior de Investigaciones Científicas, Plaza de Murillo, 2, 28014 Madrid, Spain \\ ${ }^{2}$ Departamento de Biología Vegetal y Ecología, Facultad de Biología, Sevilla, Spain \\ ${ }^{3}$ Author for correspondence (pbarbera@rjb.csic.es) \\ Communicating Editor: Jocelyn Hall
}

\begin{abstract}
A taxonomic revision of Trisetum sect. Acrospelion is presented. We include descriptions and synonyms of each taxon from a study of 670 vouchers from 45 herbaria. Detailed morphometric descriptions, illustrations, distribution maps, identification key, and habitat data are given for each taxon. Twelve names are lectotypified: Aira halleri Honck., Avena argentea Willd., Avena brevifolia Host, Avena daenensis Boiss., Avena distichophylla Vill., Trisetum argenteum var. parviflorum Parl., Trisetum cavanillesianum Borja \& Font Quer, Trisetum distichophyllum subsp. delphinense Beauverd, Trisetum macrotrichum Hack., Trisetum rigidum var. molle Somm. \& Levier, Trisetum rigidum var. ovale Somm. \& Levier, and Trisetum teberdense var. brevifolium Kharadze. We recognize seven species of Trisetum into the section, which is endemic to Eurasia: T. argenteum, T. buschianum Seredin, T. distichophyllum (Vill.) P. Beauv., T. macrotrichum, T. rigidum (M. Bieb.) Roem. \& Schult., T. tamonanteae Marrero Rodr. \& S. Scholz, and T. velutinum Boiss. Morphometric variation of the main characters is shown by box plots. The highest diversity is located in the Caucasian Mountains and the Alps, where two species were found in each area. Additionally, one species is endemic to the Canary Islands, a second to southeastern Spain, and a third to the Carpathian Mountains. In contrast with some regional treatments, T. persicum Chrtek is considered a synonym of T. rigidum (M. Bieb.) Roem. \& Schult., and T. cavanillesianum Borja \& Font Quer a synonym of T. velutinum Boiss. Trisetum rigidum subsp. teberdense (Litv.) Tzvelev and T. buschianum subsp. transcaucasicum (Seredin) Mosul. are recognized as subspecies. Vegetative propagation has been observed for the first time in specimens of T. rigidum and T. velutinum.
\end{abstract}

Keywords-Asia, Europe, grasses, lectotypification, nomenclature, taxonomy.

Trisetum Pers. [Poaceae (R. Br.) Barnhart: subfamily Pooideae Benth.] is a perennial genus of grasses which belongs to the blue grass tribe Poeae R. Br. (including tribe Aveneae Dumort.; cf. Tzvelev 1989; GPWG 2001; Soreng et al. 2003, 2007; Quintanar et al. 2007), and to subtribe Aveninae J. Presl (Soreng et al. 2015).

Trisetum is comprised of approximately 70 species that inhabit temperate and cold regions, mainly in the Northern hemisphere, but are also found in South America, Australia, and New Zealand. Typically, they live in weedy places, meadows, mountain slopes, and alpine and tundra grasslands (Hultén 1959; Chrtek 1965; Clayton and Renvoize 1986; Randall and Hilu 1986; Watson and Dallwitz 1992; Finot et al. 2004, 2005a). Detailed taxonomic revisions of American taxa, as well as taxa from New Zealand, have been made (Edgar 1998; Finot et al. 2004, 2005a, b); however, only partial revisions exist for Europe and Asia. All the species of $T$. sect. Acrospelion are endemic to this latter area.

Prior to the publication of the genus Trisetum by Persoon (1805), the species included in the genus were considered to belong to the genus Avena L. The genus Trisetaria described by Forsskål (1775) (describing only one species, Trisetaria linearis Forssk.), was considered a synonym of Trisetum by some authors (e.g. Poiret (1808), Baumgarten (1817), Dumortier (1824), Ascherson and Graebner (1899), Boissier (1854)). Others realized that Trisetaria had priority when the two genera are united (e.g. Maire (1942), Paunero (1950), Jonsell (1980)). In this study, we continue the traditional separation of the two genera based on their perennial (Trisetum) vs. annual (Trisetaria) life-cycles (e.g. Chrtek 1965, Rechinger 1970, Tzvelev 1976, Pignatti 1982, Mosulishvili 1991, Finot et al. 2004, 2005a, b); therefore, the proposal of Quintanar and Castroviejo (2010) to conserve Trisetum against Trisetaria is followed.

Trisetum has been traditionally divided into two sections: $T$. sect. Trisetum, with lax, open panicles, and culms glabrous below the inflorescences, and T. sect. Trisetaera Asch. \& Graebn., with dense, spiciform panicles, and culms pilose below the inflorescences. A review of the history of the genus can be found in Finot et al. (2005b).
Chrtek (1965) divided the Europaean species of Trisetum in four subgenera: T. subg. Distichotrisetum, T. subg. Glaciotrisetum, T. subg. Graciliotrisetum, and T. subg. Trisetum. To divide the aforementioned subgenera, Chrtek considered the most important characters to be the variation in vernation (conduplicate or convolute), sclerenchyma disposition in transverse sections of leaf blades, and the endodermal cell disposition in transverse sections of roots. Other characters considered alongside the previous ones are the innovation leaves disposition, ligule length, and rachilla hair length. The distribution of the species was also taken into account. The wide-ranging group, $T$. subg. Trisetum, was primarily characterized by convolute vernation. Trisetum flavescens and related species, T. fuscum, T. hispidum, T. macrotrichum, T. rigidum, T. spicatum, and T. velutinum belong to this subgenus, along with T. agrostideum (Laest.) Fr., T. alpestre (Host) P. Beauv., T. baregense Laffitte \& Miégev., T. burnoufii Req. ex Parl., and T. sibiricum Rupr. The other subgenera have fewer species and narrower distributions. Trisetum subg. Distichotrisetum, comprising T. distichophyllum and T. argenteum, was characterized by having conduplicate vernation, leaves with sclerenchyma clearly developed on young leaves, endodermal cells of roots C- and U-shaped, and young leaves distichously disposed. Another species, T. glaciale, endemic to the Sierra Nevada in southeastern Spain, belongs to $T$. subg. Glaciotrisetum, displaying the conduplicate vernation, endodermal cells of roots C- and U-shaped, and leaf blades with sclerenchyma only present on the abaxial side of the central vascular bundle as a well-developed girder, and a cap of sclerenchyma at the margin. The last group, T. subg. Graciliotrisetum is made up of one species from Corsica and Sardinia, T. gracile, characterized by vernation conduplicate to convolute, endodermal cells of roots O-shaped, and leaf blades with sclerenchyma adaxially and abaxially disposed in an arched well-developed strand.

Chrtek (1965) also divided the subgenus Trisetum in five sections: T. sect. Carpatica, T. sect. Hispanica, T. sect. Rigida, T. sect. Trisetaera, and T. sect. Trisetum. 
Chrtek (1965) proposed an extremely heterogeneous section endemic to the Iberian Peninsula, T. sect. Hispanica (comprising the type species T. velutinum, and T. hispidum), which shares elongated, rectangular long cells with slightly sinuous lateral walls, hirsute leaves, and inconspicuous bulliform cells. The monotypic T. sect. Carpatica, established for T. fuscum of the Carpathian Mountains, has slightly longer ligules than those found in T. sect. Hispanica, and hairy palea keels. In Trisetum sect. Rigida, Chrtek (1965) included T. macrotrichum and $T$. rigidum, characterized by long rachilla hairs and distichous disposition of young leaves (sclerenchyma disposition and epidermal cells of abaxial side of innovation leaves as in $T$. sect. Trisetum). Trisetum sect. Trisetaera includes the T. spicatum complex with contracted panicles, and leaf blades with sclerenchyma adaxially and abaxially disposed in an arched, welldeveloped strand, as T. subg. Glaciotrisetum. Trisetum sect. Trisetum is separated from $T$. sect. Trisetaera by its lax panicles and leaves with the sclerenchyma clearly developed in young leaves. Chrtek (1965) noted that T. agrostideum, which belongs to $T$. sect. Trisetum, has a sclerenchyma disposition similar to $T$. sect. Trisetaera.

Chrtek (1967) described one new species from Greece, Trisetum rechingeri Chrtek, and divided sect. Rigida into three new series: T. ser. Macrotricha, T. ser. Laconica Chrtek, and T. ser. Rigida. Trisetum ser. Macrotricha, including only T. macrotrichum, was diagnosed by its long callus hairs (reaching to $1 / 2$ of the lemma) and long innovation leaves, while T. ser. Laconica had shorter rachilla and callus hairs, and shorter innovation leaves. Trisetum ser. Rigida, including T. buschianum, T. daenense, $T$. rigidum, $T$. teberdense, and $T$. transcaucasicum, was characterized by its long rachilla hairs (reaching to $3 / 4$ of the lemma), short callus hairs, and short or long culm innovation leaves. Chrtek (1968) in his study of some Asian taxa, described one species (T. persicum Chrtek) and one subspecies (T. rigidum subsp. demavandense Chrtek), both from Iran, in T. ser. Rigida.

Tzvelev (1976) simplified Chrtek's classification recognizing three sections ( $T$. sect. Rigida, $T$. sect. Trisetaria, and $T$. sect. Trisetum) in his treatment of Trisetum for the Soviet Union. He characterized the different sections by the type of growth of underground and vegetative shoots, the leaf disposition, panicle density, and the rachilla and callus hair length. He did not account for the anatomical characters used by Chrtek. Tzvelev included four species and two subspecies within $T$. sect. Rigida, and avoided discussion of the series of $T$. sect. Rigida. In the section, Tzvelev included plants with long creeping underground shoots, long vegetative shoots, with distant nodes and distinctly distichous leaves, panicles sparse or fairly dense, with somewhat scabrous (to almost smooth) branches, rachillas with hairs $2-4.2 \mathrm{~mm}$ long, callus hairs 0.6-3.5 mm long, and anthers $1.7-3 \mathrm{~mm}$ long.

Chrtek $(1965,1967)$ overlooked the name Acrospelion, coined by Roemer and Schultes (1827) for a group of Trisetum. This genus was later combined as a section by Pfeiffer (1871-1873) and typified based on Trisetum distichophyllum. Therefore, because of its earlier date, the epithet Acrospelion has priority over the epithet Rigida in the sectional rank.

This work, together with that of Chrtek, provides the most comprehensive study on this section to date. However, the complex and extremely analytical Chrtek subgeneric divisions proved unsatisfactory and impractical due to the overlap of some characters among his proposed groups. Consequently, we prefer to follow Tzvelev's outline, and recognize a broader and better characterized T. sect. Acrospelion, without resorting to anatomical characters. Therefore, T. sect. Acrospelion includes seven species, $T$. argenteum, $T$. buschianum, $T$. distichophyllum, T. macrotrichum, T. rigidum, T. tamonanteae, and $T$. velutinum. Trisetum sect. Acrospelion is here characterized by having young shoots with distichous disposition, more or less rigid, and also loosely tufted habit, panicles from lax to slightly dense, glumes unequal to subequal, and long hairs on the rachilla segments between first and second floret and callus.

We present a taxonomic revision of Trisetum sect. Acrospelion based on the careful review of herbarium material, as a first installment of a monograph of Trisetum in Eurasia. For technical reasons, and the large number of species in the genus, the work will be presented in various articles, of which this is the first.

\section{Materials ANd Methods}

This revision is based on the study of 670 herbarium specimens from the following herbaria: A, ABH, ARAN, B, BC, BCN, BERG, BM, BP, C, F, FI, G, GB, GDA, GH, GOET, H, HBG, IBF, JE, K, L, LD, LE, LPA, M, MA, MO, MPU, MW, NY, O, P, PH, PR, S, SEV, TBI, UPS, US, VAL, W, WIS, and WU (acronyms according to Thiers 2017). An index of specimens cited is provided in Appendix 1.

One hundred and twenty specimens were included for the morphometric analysis, as operational taxonomic units (OTUs), selected to represent as far as possible the geographic range and morphological variability of the taxa. Fifty-nine quantitative characters, listed in Appendix 2, were recorded using a Mitutoyo CD-15DCX digital vernier caliper on 20 specimens of each species when available. Commonly used characters in Trisetum taxonomy were selected, as well as those observed to be variable and diagnostic in herbarium specimens. Spikelet measurements were taken on the distal spikelet from the longest branch of the second node of the panicle; floret measurements were taken from the proximal branches. Leaf blade width was measured one $\mathrm{cm}$ above the ligule insertion. The segment between the first and second flower is also referred to as the rachilla. Each character was analyzed for its minimum, maximum, and lower and upper quartiles, using the STATISTICA package (www.statsoft.com). Quantitative and qualitative characters were used to build an identification key and descriptions. Minimum and maximum values are noted in parentheses, and the lower and upper quartile values are noted outside. A combination of morphological characters was employed to distinguish species. Transverse sections of top leaf blades were prepared by hand, stained with Fasga (Tolivia and Tolivia 1987), and photographed with a Nikon SMZ1000 optical microscope. For leaf anatomy, the terminology defined by Ellis $(1976,1979)$ was used.

Additional data on the habitat, distribution, and chromosome numbers was checked from literature and collection labels. Chromosome numbers were summarized from the literature, but the extant vouchers, if any, were not revised. The list of numbered collections, examined specimens, and coordinates used to produce distribution maps were generated by a Microsoft ACCESS database (Microsoft, Seattle, Washington) using ArcGis v.9.3 (ESRI 2008). The material studied is listed below each species. The data for establishing coordinates was completed in almost all cases, using the www.geonames.org database.

\section{Results}

Morphology-Haвiт - The species of Trisetum sect. Acrospelion are perennial grasses with short rhizomatous or stoloniferous rhizomes, with well-isolated shoots or loosely tufted habit (densely tufted in T. tamonanteae). They are also characterized by their young shoots with distinctly distichous leaf disposition, also noticeable in older leaves. Most of the species present intravaginal growth in the basal part, although occasionally extravaginal growth occurs in the upper nodes (with the exception of T. rigidum and sometimes T. buschianum, whose nodes are never enclosed by the sheaths).

VEGETATIVE BODY - The culms are straight, with (3)4-10 nodes, and usually shorter than $60 \mathrm{~cm}$. The variation in size 
among the species of this section is smaller in those with more limited biogeographical ranges; $T$. rigidum is the most widespread species of the section, ranging from (18.5-)37 to $57(-76.3) \mathrm{cm}$ in height. Culms are glabrous in most of the species, pubescent in T. tamonanteae, and slightly puberulous around the nodes in $T$. rigidum, or occasionally puberulous in the upper internode below the panicle in T. macrotrichum and T. velutinum.

LEAVES - The indumentum, shape, and size of ligules, leaf sheaths, and leaf blades are variable characters in the same plant, depending on their location on the plant, whether they are basal or the top culm leaf. There is also variation according to leaf length and age. Young leaf blades are usually less hairy than mature ones. Therefore, notable heterophylly occurs between young and mature leaf blades in T. sect. Acrospelion, as well as in the closely related genus Koeleria (Quintanar and Castroviejo 2013).

Leaf SHeATHs-Basal leaf sheaths are puberulous to pubescent, and rarely glabrous (velutinous in T. velutinum). Top culm leaf sheaths can be shorter or longer than their respective internodes, and are always glabrous (except in T. tamonanteae, which has pubescent to densely pubescent sheaths, sometimes puberulous in T. velutinum). Sheath margins are glabrous, except for those of T. macrotrichum, which are ciliate (rarely ciliate in $T$. distichophyllum).

LEAF BLADES - The species of Trisetum sect. Acrospelion have flat to inrolled leaf blades. Hermann (1956) classified both $T$. distichophyllum and T. argenteum as a different subgenus (T. subgen. Argentaria Hermann), because of the presence of young conduplicate leaf blades. Chrtek (1965), using additional morphological and anatomical characters, divided those species with conduplicate or convoluted vernal leaf blades into four separate subgenera (subgen. Trisetum, Distichotrisetum, Glaciotrisetum, and Graciliotrisetum). The indumentum of leaf blades has traditionally been used as a distinctive character for taxon delimitation (Tzvelev 1976; Jonsell 1980; Pignatti 1982). However, leaf blade features (mainly indumentum and width) have been considered less important than those linked to spikelet morphology for the taxonomy of species with large distribution (i.e. T. rigidum) due to the high variability of leaf blade traits, which are more important at the subspecific level (i.e. T. rigidum and T. buschianum). Most of the species are abaxially and adaxially puberulous to pubescent, with hairs also found on the margin, except for T. rigidum, which has been separated into two subspecies mainly because of the indumentum and size of leaf blades: glabrous in $T$. rigidum subsp. rigidum, and with long hairs along the margin and wider leaf blades in T. rigidum subsp. teberdense (Fig. 1A-B).

All of the species of Trisetum sect. Acrospelion are $\mathrm{C}_{3}$ $(\mathrm{XyMS}+)$ and mesophytic or xerophytic grasses. While the abaxial surface of the leaf blades often has a regular outline, the adaxial surface has ribs of varying depth, and the midrib is not readily distinguishable (Metcalfe 1960; Watson and Dallwitz 1992). The depth of the intercostal zones and associated ribs is more pronounced in xerophytic species. Chrtek (1965) conducted the first detailed study on the histology of European Trisetum. He used the distribution of sclerenchyma strands or girders along the young leaf blades as a main taxonomical character in his subgeneric classification. Among the species of this section, all the vascular bundles are accompanied by more or less developed sclerenchyma girders and strands that attach to both the abaxial and adaxial sides of the leaf blade. Those species from strongly xeric habitats have well-developed sclerenchyma strands, specifically on the abaxial side where the ribs are less developed. Thus, T. velutinum, T. rigidum, and T. argenteum, all from more xerophytic habitats, have wider strands of sclerenchyma, whereas T. macrotrichum, T. distichophyllum and T. buschianum have thinner strands. This layer is discontinuous and narrows at the adaxial surface, and it is often interrupted at the furrows.

LigulEs - The shape and size of ligules are variable, with those of basal leaves being shorter than those of top culm leaves. The ligule measurements used in this study were taken from the top culm leaf blades. Trisetum rigidum has the longest ligules $(3.4(-4.2) \mathrm{mm})$, while $T$. argenteum has the shortest $(0.3-0.5(-0.7) \mathrm{mm})$ (Fig. 1C). The ligules are membranous, with a truncate, laciniate to slightly laciniate, sometimes denticulate apex, glabrous or almost glabrous, with some hairs in most of the species (mostly pubescent in T. argenteum, T. distichophyllum, and T. velutinum).

INFLORESCENCES - Inflorescences are paniculate, from lax to dense in T. tamonanteae, oblong to elliptic or lanceolate in outline, and sometimes ovate in $T$. distichophyllum and $T$. buschianum subsp. transcaucasicum. Trisetum rigidum and $T$. macrotrichum have the longest basal branches and panicles. Trisetum sect. Acrospelion has a glabrous to slightly pubescent rachis, whereas $T$. tamonanteae has a pubescent rachis with hairs up to $0.6 \mathrm{~mm}$ long.

Vegetative propagation has been observed in some specimens of Trisetum rigidum and $T$. velutinum for the first time (Fig. 8); up until these observations, T. tamonanteae was the first species in the genus Trisetum in which this type of propagation was observed (Marrero Rodríguez and Scholz 2013).

SPIKELETS-The size of the spikelets of Trisetum sect. Acrospelion varies according to the lemma length, being slightly longer, compared to the rest of species, in T. rigidum, and slightly shorter in T. argenteum and T. tamonanteae. For convenience, the awn is not included in the length of the spikelet. The variation in the number of florets, usually 2-3 per spikelet, is significant. Trisetum macrotrichum and T. tamonanteae have up to 4 and 5 florets per spikelet respectively.

Rachilla segments are always pubescent, with long white hairs between the first and second floret in most of the species, whereas they are shorter in T. tamonanteae, T. velutinum, and $T$. argenteum, with hairs that are $0.1-0.3(-0.7) \mathrm{mm}$ long, (0.3-)0.7-1.6(-2.4) mm long, and (1-)1.7-2.5(-3.4) $\mathrm{mm}$ long, respectively (Fig. 1E). The length of these hairs can be useful as a diagnostic character.

GLUMES-Glumes are always unequal in Trisetum argenteum, T. macrotrichum, and T. rigidum, and sometimes subequal in $T$. buschianum, T. distichophyllum, T. tamonanteae, and T. velutinum. Lower and upper glumes are acute to long acuminate, and glabrous, with some short hairs from the middle part to the top of the main nerve and along the margins. The lower glume is always shorter than the upper one, and usually has a main nerve reaching the tip, sometimes having one or two very short lateral nerves, extending at most to the middle part of the glume (Fig. 1D). The upper glume always has three nerves, the central one reaching the tip like the lower glume, with the lateral ones more notable and only extending to the upper half.

Lemma - The lemma of the species of Trisetum sect. Acrospelion, as well as that of the rest of the genus, is characterized by having a dorsal awn and a bifid apex with two apical teeth normally ending in two aristules separated by a more or less deep sinus. Aristules are the main lateral nerves protruding 
A

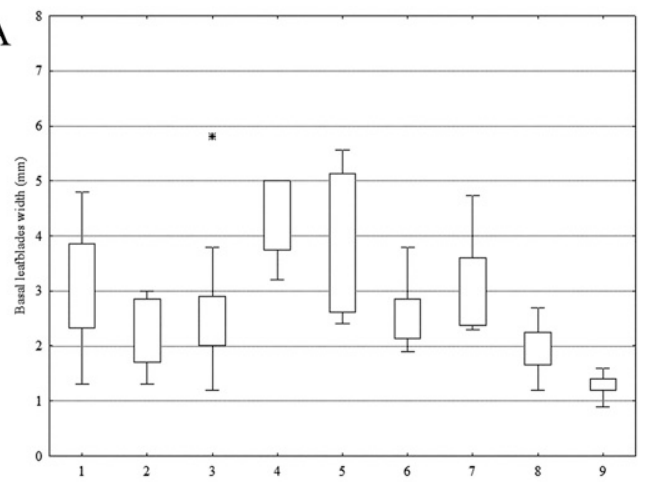

C

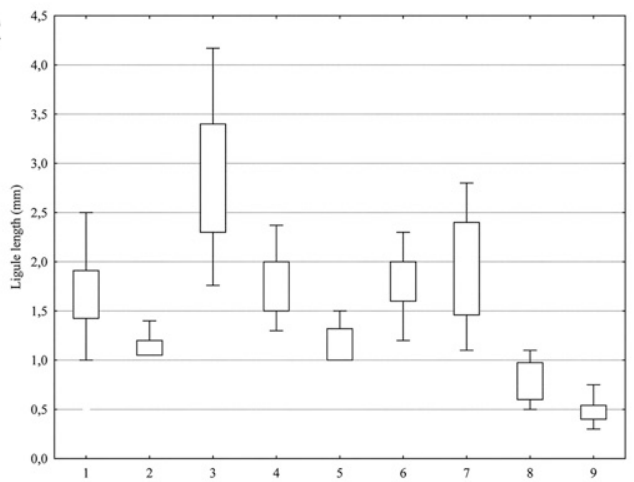

E

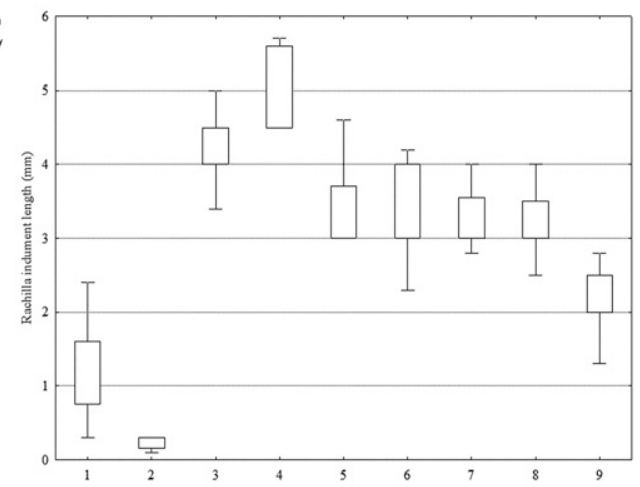

G

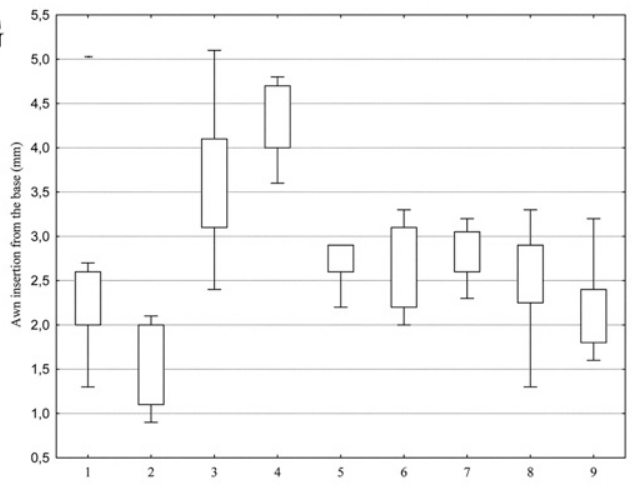

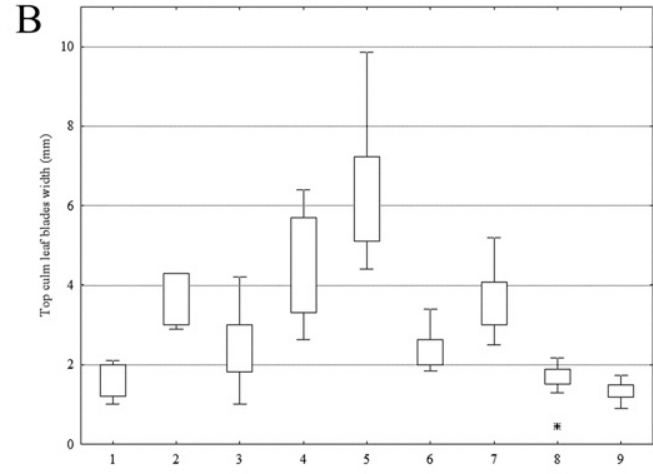

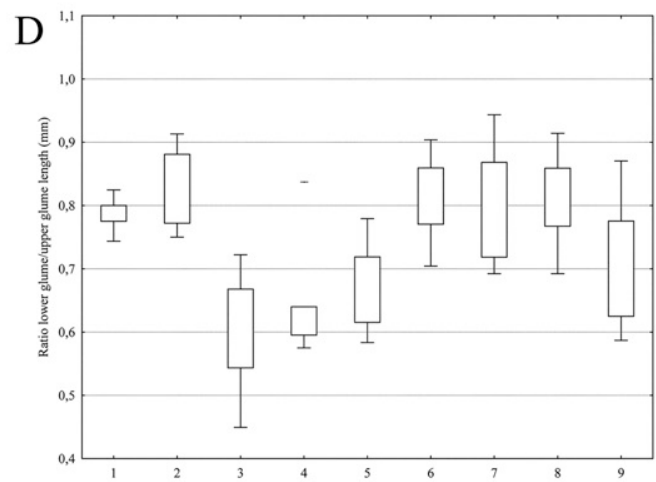

F

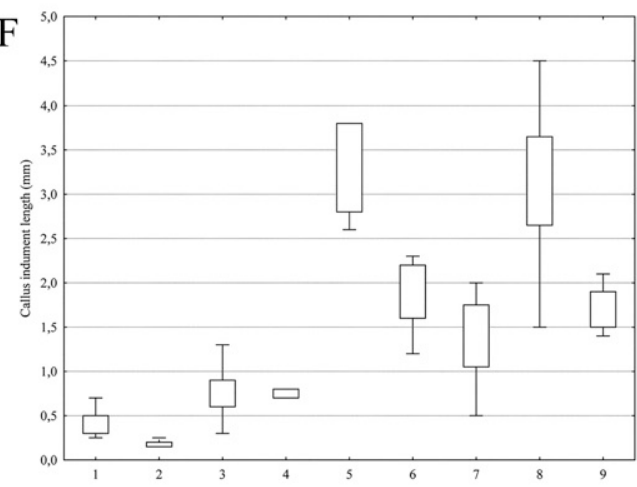

$\mathrm{H}$

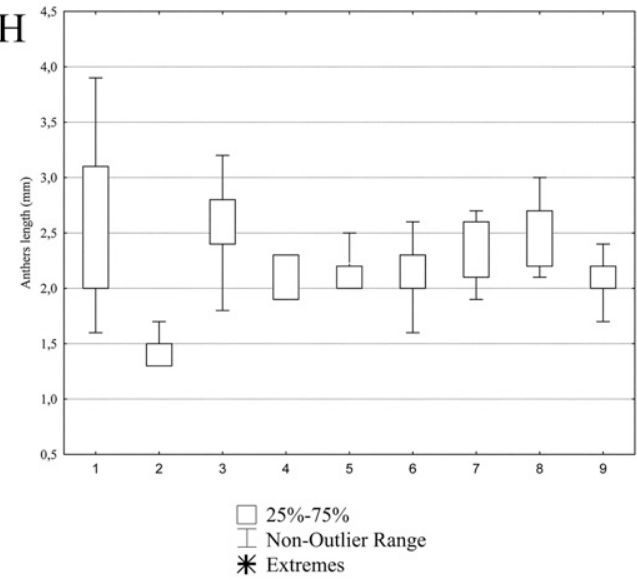

FIG. 1. Box plots of a selection of studied variables. 1) T. velutinum. 2) T. tamonanteae. 3) T. rigidum subsp. rigidum. 4) T. rigidum subsp. teberdense. 5) T. macrotrichum. 6) T. buschianum subsp. transcaucasicum. 7) T. buschianum subsp. buschianum. 8) T. distichophyllum. 9) T. argenteum.

through each tooth apex (Nicora 1978; Koch 1979; Finot et al. 2006). Apical teeth and aristules are variable characters at the species level. The length of the lemma, without taking the awn into account, is a good diagnostic character, being shorter in
T. tamonanteae and T. argenteum ((2.6-)3-4.3(-5.4) $\mathrm{mm}$ and (4-)4.4-5.2(-6.5) $\mathrm{mm}$ long, respectively) and longer in $T$. rigidum subsp. rigidum and $T$. rigidum subsp. teberdense ((5.3-)6.4-7.7(-9.3) and (6.7-)7-8.5 mm long, respectively). 
The lemma is laterally compressed, from narrowly or broadly lanceolate to elliptic or oblong, with a glabrous to scabridulous or strigulous surface, having slightly longer hairs on the upper part.

CAllus - The callus has an oblong to elliptic shape, sometimes rounded, always with hairs in the species of this section. The length of the callus indumentum is an important taxonomic character, being longer in T. distichophyllum and T. macrotrichum ((1.5-)2.2-3.7(-4.5) and (2.6-)2.8-3.8 mm long, respectively) and shorter in $T$. tamonanteae $(0.1-0.3 \mathrm{~mm}$ long), $T$. velutinum $(0.25-0.5(-0.7) \mathrm{mm}$ long) and $T$. rigidum subsp. rigidum and $T$. rigidum subsp. teberdense ((0.3-)0.6-0.9(-1.4) and 0.7-0.8(-1) $\mathrm{mm}$ long, respectively) (Fig. 1F).

PALEA - The palea is enclosed by the margins of the lemma, opening only during anthesis. The palea is a hyaline structure, with two keels extruding into two fine teeth, with very short hairs, also present along the outer edges. The length of both the palea and the lemma is unequal to subequal, being slightly less similar in $T$. tamonanteae and $T$. rigidum (ratio palea length/lemma length (0.56-)0.63-0.74 and (0.54-) $0.66-0.81(-0.87)$, respectively).

LODICULES - The two lodicules flank the dorsal sides of the ovary or caryopsis, having a more or less oblanceolate shape. The apex is irregular to regularly lobulate (2-4-lobate) or denticulate, and glabrous, rarely with scattered hairs in $T$. velutinum.

Awn-The most important taxonomic character of the awn is length and the point of insertion on the lemma. The awn shape depends upon the maturity of the specimen, but in general, it is more or less geniculate and twisted near the base, although almost straight in T. tamonanteae. This species has the shortest awn ((2.8-)3.4-5.1(-6.1) mm), but the length does not differ greatly in the rest of the species of the section. In $T$. tamonanteae, T. velutinum, and T. argenteum, the point of insertion is closer to the base of the lemma, compared to the rest of the species. Trisetum rigidum is the species with the most apical awn (Fig. 1G).
STAMENS - The length of the anthers is fairly constant at the species level. Trisetum tamonanteae is the species with the smallest anthers (0.8-1.6(-1.8) mm long) (Fig. 1H).

OVARY AND CARYOPSIS - The ovary and caryopsis are very similar in all species, being glabrous, sometimes slightly puberulous on the apex in T. rigidum. The mature caryopsis is oblong in shape, narrowly elliptic in transverse section, not sulcate, with punctiform hilum. The endosperm is liquid.

\section{TAXONOMiC TREATMENT}

Trisetum sect. Acrospelion (Besser) Pfeiff., Nomencl. Bot. 1(1): 38. 1877. Acrospelion Besser, in Schult. \& Schult. f., Mant. 3 (Add. 1): 526 ["326"]. 1827. T. [unranked] Acrospelion (Besser) Trin., Mém. Acad. Imp. Sci. St.-Petersbourg, sér. 6, Sci. Math. 1(1): 59. 1830.—LECTOTYPE: T. distichophyllum (Vill.) P. Beauv. (designated by Pfeiffer 1877: 38).

T. sect. Rigida Chrtek, Bot. Not. 118(2): 222. 1965. T. ser. Rigida Chrtek, Acta Univ. Carol., Biol. 1966(2): 94. 1967. T. subsect. Rigida (Chrtek) Mosul., Zametki Sist. Geogr. Rast. 42: 33. 1991.-TYPE: T. rigidum (M. Bieb.) Roem. \& Schult.

T. subg. Argentaria F. Herm., Fl. N.-Mitt.-Eur., 120, Stuttgart. 1956. T. subg. Distichotrisetum Chrtek, Bot. Not. 118(2): 223. 1965., nom. illeg.-TYPE: T. distichophyllum (Vill.) P. Beauv.

T. sect. Hispanica Chrtek, Bot. Not. 118(2): 222. 1965.-TYPE: T. velutinum Boiss.

T.ser. Macrotricha Chrtek, Acta Univ. Carol., Biol. 1966(2): 94. 1967.-TYPE: T. macrotrichum Hack.

T. subsect. Monticolae Mosul., Zametki Sist. Geogr. Rast. 42: 33. 1991.-TYPE: T. buschianum Seredin.

Herbs with or without loosely tufted habit (rarely densely tufted); young shoots with distichous disposition, \pm rigid; panicles from lax to slightly dense (rarely dense); glumes unequal to subequal; rachilla segments between first and second flower and callus usually with long hairs.

\section{Key to the Species of Trisetum Sect. Acrospelion}

1. Top culm leaf blades generally reaching or surpassing the panicles; rachilla segments between first and second flower with hairs $0.1-0.3(-0.7) \mathrm{mm}$ long;

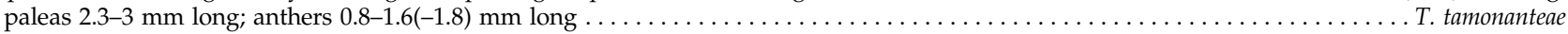

1. Top culm leaf blades not or rarely reaching the panicle; rachilla segments between first and second flower with hairs $(0.3-) 0.7-4.7(-5.7)$ mm long; paleas

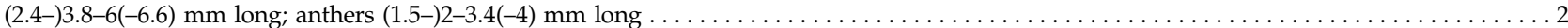

2. Sheaths velutinous; rachilla segments between first and second flower with hairs $(0.3-) 0.7-1.6(-2.4) \mathrm{mm}$ long ............ T. velutinum

2. Sheaths glabrous or hairy but not velutinous; rachilla segments between first and second flower with hairs $(1-) 3-4.2(-5.7) \mathrm{mm}$ long ....... 3 3. Rachilla segments between first and second flower with hairs (3.2-)4-4.7(-5.7) $\mathrm{mm}$ long; callus hairs $(0.3-) 0.6-0.9(-1.4) \mathrm{mm}$ long ..... 4 4. Plants (47-)50-67(-72) cm tall; basal leaf blades (5-)7.5-8 (-8.6) cm long, not rigid, with hairs along the margins $0.4-1 \mathrm{~mm}$ long; top culm leaf blades usually erect, sometimes reaching into the panicle $\ldots \ldots \ldots \ldots \ldots \ldots \ldots \ldots \ldots \ldots \ldots \ldots$ T. rigidum subsp. teberdense

4. Plants (18.5-)34.3-56.5(-76.3) cm tall; basal leaf blades $(0.8-) 2.8-5.2(-7) \mathrm{cm}$ long, rigid, with hairs along the margins $0.05-0.6(-1) \mathrm{mm}$ long; top culm leaf blades divergent (rarely erect), not reaching into the panicle .................... T. rigidum subsp. rigidum

3. Rachilla segments between first and second flower with hairs (1)2.5-3.5(4.8) $\mathrm{mm}$ long; callus hairs (0.5-) $1.5-2.9(-4.5) \mathrm{mm}$ long ....... 5 5. Plants (33-)38.5-67(-77) cm tall; top culm leaf blades $(4.4-5-7.2(-10) \mathrm{mm}$ wide. ..................... T. macrotrichum

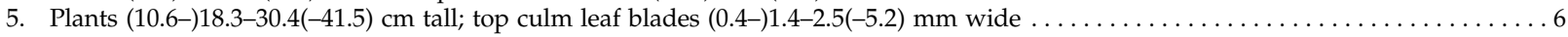
6. Habit slender; basal leaf blades flat to inrolled, $(0.9-) 1.2-1.5(-1.7) \mathrm{mm}$ wide $\ldots \ldots \ldots \ldots \ldots \ldots \ldots \ldots \ldots \ldots \ldots$. argenteum

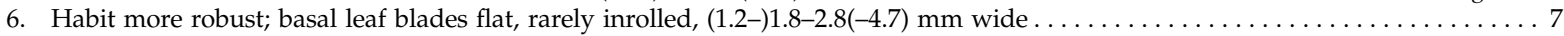
7. Ligules $0.5-1 \mathrm{~mm}$ long, from densely to slightly pubescent on the surface and apex, sometimes glabrous; callus hairs

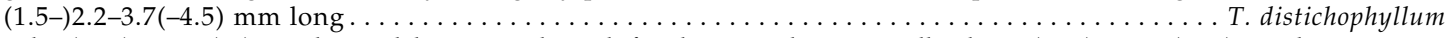
7. Ligules (1.2-)1.5-2.1(-3) mm long, glabrous, rarely with few hairs on the apex; callus hairs (1.2-)1.6-2.2(-2.3) mm long. ...8 8. Plants (17-)23.7-30.7(-35) cm tall; nodes separated along the culm; top culm leaf sheaths $(6-) 6.7-9(-10) \mathrm{cm}$ long ......

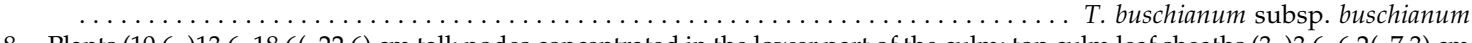
8. Plants (10.6-)13.6-18.6(-22.6) cm tall; nodes concentrated in the lower part of the culm; top culm leaf sheaths (3-)3.6-6.2(-7.3) cm

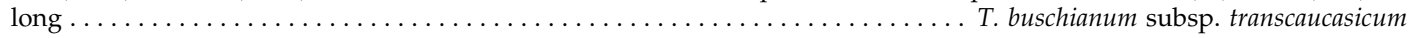


1. Trisetumdistichophyllum (Vill.) P. Beauv., Ess. Agrostogr.: 88. 1812. Avena distichophylla Vill., Prosp. Hist. Pl. Dauphiné: 16. 1779. Avena disticha Lam., Encycl. 1: 333. 1783., nom. superfl. Trisetaria distichophylla (Vill.) Paunero, Anales Jard. Bot. Madrid 9: 514. 1950.-TYPE: FRANCE. Dauphiné, $45^{\circ} 0^{\prime} \mathrm{N}, 5^{\circ} 15^{\prime} \mathrm{E}, \mathrm{D}$. Villars s. $n$. (lectotype, here designated, P-LA-564210!).

Aira halleri Honck., Verz. Gew. Teutschl.: 212. 1782.-TYPE: [unknown locality]. V.A. Haller s. n. (lectotype, here designated, P-Haller (image!); choice made by M. Kerguelen in sched.).

Avena brevifolia Host, Icon. Descr. Gram. Austriac. 3: 28, Table 40. 1805. Trisetum brevifolium (Host) Roem. \& Schult., Syst. Veg., 2: 665. 1817. Trisetaria brevifolia (Host) Baumg., Enum. Stirp. Transsilv., Mant.: 80. 1846. Trisetum distichophyllum subsp. brevifolium (Host) Pignatti, Giorn. Bot. Ital. 111(1-2): 59. 1977. Trisetaria distichophylla subsp. brevifolia (Host) Banfi \& Soldano, Atti Soc. Ital. Sci. Nat. Mus. Civico Storia Nat. Milano 135: 382. 1996.-TYPE: AUSTRIA. Host herbarium, without locality or collector (lectotype, here designated, W-1885-0002402!).

Avena distichophylla var. gracilis Shuttlew., Mag. Zoo. Bot. 2: 17. 1838.-TYPE: SWITZERLAND. Valais, M. Gemmi, $46^{\circ} 23^{\prime} \mathrm{N}, 7^{\circ} 36^{\prime} \mathrm{E}, 30$ Aug 1836, R.J. Shuttleworth s. n. (no original material found).

T. albanicum Jáv., Magyar Bot. Lapok 18: 1. 1920. T. distichophyllum subsp. albanicum (Jáv.) Hayek, Feddes Repert., Beih. 30(3): 313. 1932.-TYPE: ALBANIA. Hekurave Mountains, Djakova, Bunjaj, cacumine Stüla Gris, $42^{\circ} 23^{\prime} \mathrm{N}, 1^{\circ} 58^{\prime} \mathrm{E}, 24$ Aug 1918, S. Jávorka s. $n$. (lectotype, designated by Kováts 2000: 35, BP!).

T. distichophyllum subsp. delphinense Beauverd, Bull. Soc. Bot. Genève, sér. 2, 26: 122, Fig. 1. 1934.-TYPE: FRANCE. Hautes-Alpes, Monétier, 445'ㅅN, 6³2'E, 25 Jul 1933, G. Beauverd s. n. (lectotype, here designated, G-386040 (image!); isolectotype, G-386041 (image!)).

Herbs (14.8-)17.8-27.2(-41.5) cm tall, loosely tufted, short rhizomatous, culms (0.3-)0.4-0.6(-0.7) $\mathrm{mm}$ diam, glabrous; nodes $4-5(-7)$, concentrated in lower part of the culm, enclosed by the sheaths, sometimes the upper one exposed, glabrous. Basal leaf sheaths pubescent, sometimes with longer hairs on the upper margins up to $0.1 \mathrm{~mm}$ long, decaying into fibers, brownish to yellowish, sometimes greenish; basal leaf blades (1-)1.6-3.4(-7.7) $\mathrm{cm}$ long $\times(1.2-)$ 1.6-2.2(-3.8) mm wide, markedly distichous, flat to slightly inrolled, sometimes conduplicate, from densely to slightly pubescent or scabridulous adaxially, slightly pubescent to glabrous abaxially, with hairs (0.05-)0.1-0.3(-1.3) $\mathrm{mm}$ long, greyish to greenish; top culm leaf sheaths (3.3-)4.3-7(-10.8) $\mathrm{cm}$ long, shorter than the internodes, sometimes longer or almost equal, glabrous, without cilia on the margins, or rarely with cilia; top culm leaf blades $(0.5-) 1.2-2.6(-6) \mathrm{cm}$ long $\times$ (0.4-)1.5-1.9(-2.5) mm wide, conduplicate to inrolled, sometimes flat, adaxially pubescent to densely pubescent, abaxially pubescent to slightly pubescent, rarely almost glabrous, with hairs $0.05-0.1(-0.3) \mathrm{mm}$ long along the margins, greenish to greyish; inner collar region glabrous to slightly pubescent, usually with scattered hairs on the margins $0.05-0.2$ (-1.5) mm long; ligules $0.5-1 \mathrm{~mm}$ long, laciniate to slightly laciniate, rarely slightly lobulated, from densely to slightly pubescent with hairs $0.05-0.2(-0.3) \mathrm{mm}$ long on the abaxial surface and apex, sometimes glabrous. Basal node of the panicle glabrous. Panicles (2.8-)4-6.3(-9.4) cm long $\times(1.2-) 1.6-2.5$ $(-4.3) \mathrm{cm}$ wide, ovate to broadly lanceolate in outline, sometimes oblong-elliptic, lax, rarely dense, with most rachis internodes plainly visible; rachis glabrous to slightly puberulous (mostly on the upper part), with hairs $0.05-0.15 \mathrm{~mm}$ long; longest basal branches (0.5-)0.6-1.8(-3.2) cm long. Spikelets (5.5-)7-8(-8.6) mm long $\times(2-) 2.7-4.8(-7) \mathrm{mm}$ wide, 2-3flowered; pedicels (1.8-)2.7-4.2(-5) mm long, slightly pubescent with hairs $0.05-0.2 \mathrm{~mm}$ long, sometimes glabrous with scattered hairs distally. Glumes unequal to subequal (ratio lower glume length/upper glume length $=(0.69-) 0.77-0.86(-1))$; lower glume (3.6-)5-6.4(-7) mm long $\times(0.8-) 1.1-1.6(-2) \mathrm{mm}$ wide, narrowly to broadly lanceolate, sometimes elliptic or oblong (ratio lower glume width/lower glume length $=(0.15-)$ $0.22-0.26(-0.3))$, acuminate to long acuminate, sometimes acute, (1-)2-3-nerved, glabrous, sometimes with short hairs up to $0.1 \mathrm{~mm}$ long from the center to the upper part of the main nerve, purplish to greenish, with margins hyaline; upper glume (5.2-)6.6-7.3(-8.1) mm long $\times(1.4-) 1.8-2.2(-2.6) \mathrm{mm}$ wide, elliptic to oblong, sometimes lanceolate, rarely slightly oblanceolate (ratio upper glume width/upper glume length $=$ $(0.23-) 0.26-0.31(-0.4))$, acuminate to long acuminate, rarely acute, 3-nerved, glabrous, usually with hairs up to $0.1 \mathrm{~mm}$ long, from the center to the upper part of the central nerve, purplish to greenish on the central part, margins mostly hyaline; rachilla segments between first and second floret (0.7-)1-1.3(-1.6) $\mathrm{mm}$ long, with hairs (2-)3-3.8(-4.8) $\mathrm{mm}$ long; rachilla segments to sterile floret $(0.7-) 1.3-2(-2.3) \mathrm{mm}$ long, with hairs (1.4-)1.8-2.7(-3.5) mm long. Lemmas (4.5-)5.7-6.4(-7.5) mm long $\times(0.8-) 1.1-1.6(-2) \mathrm{mm}$ wide, elliptic to broadly lanceolate, rarely oblong (ratio lemma width/lemma length $=(0.2-) 0.26-0.32(-0.4))$, scabridulous, with hairs $0.05-0.1(-0.8) \mathrm{mm}$ long, the longest ones on the central part and on the awn insertion, greenish to yellowish on the central part, surrounded by purple; apical teeth absent or $0.2-0.4(1.2) \mathrm{mm}$ long, with aristules (0.1-)0.2-0.4(-0.7) mm long; awn (4-)5.5-6.6(-8) $\mathrm{mm}$ long, inserted (1.3-)2.3-3(-3.3) $\mathrm{mm}$ from the base (ratio awn insertion from the base length/lemma length $=(0.2-) 0.4-0.5)$, geniculate and twisted near the base, sometimes straight, with very short adpressed hairs up to $0.15 \mathrm{~mm}$ long; callus $0.2-0.4(-0.5) \mathrm{mm}$ long, oblong to elliptic, with hairs (1.5-)2.2-3.7(-4.5) mm long. Paleas (3.8-)4.5-5.4(-6.6) $\mathrm{mm}$ long $\times(0.8-) 1-1.3(-1.6) \mathrm{mm}$ wide (ratio palea length/lemma length $=(0.7-) 0.78-0.87(-1))$, elliptic to narrowly elliptic or oblong, with hairs from the center to the upper part of the outer edges; keels with short hairs mostly on the apex, ending in teeth $0.1-0.3(-0.4) \mathrm{mm}$ long. Lodicules (0.6-)0.7-1(-1.2) $\mathrm{mm}$ long, with apex regularly or irregularly lobate, sometimes with a lateral linear lobe. Anthers 2.1-2.7(-3) mm long. Ovary (0.3-)0.6-1(-1.3) mm long, glabrous. Caryopsis $1.8-3 \mathrm{~mm}$ long $\times 0.4-0.8 \mathrm{~mm}$ wide. Figure 2 .

Chromosome Number-2n = 28, 56 (Beuret 1974; Lippert 2006).

Phenology-Flowering from June to September.

Distribution and Habitat-Trisetum distichophyllum occurs in the Alps, from west to east, reaching to the Albanian Alps between Montenegro and Albania; at 950-2,700 m elevation; around rocks and pebbles, mostly in calcareous soils, also in granites. Figure 3.

Specimens Examined-ALBANIA. Kukës County: Tropojë district, on the northern slope of mt. Maja e Ershelit, above village Peraj, $42^{\circ} 29^{\prime} \mathrm{N}$, 


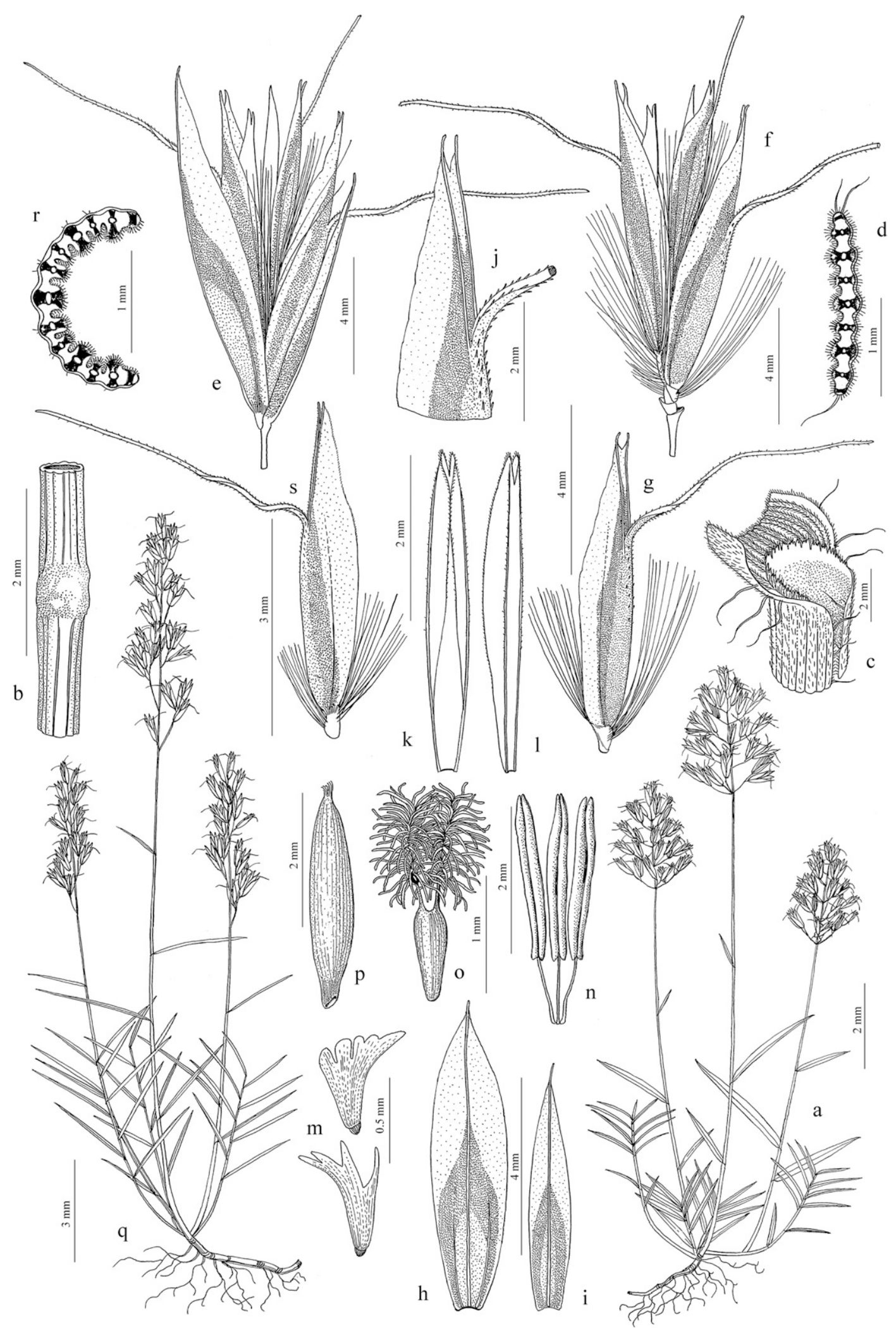

Fig. 2. A-P. Trisetum distichophyllum. A. Habit. B. Portions of the culm and node. C. Sheath, ligule, and portion of the blade. D. Transverse section of leaf blade. E. Spikelet. F. Florets. G. Floret. H. First glume, dorsal view. I. Second glume, dorsal view. J. Lemma, upper part, lateral view. K. Palea, ventral view. L. Palea, dorsal view. M. Lodicules. N. Stamens. O. Pistil. P. Caryopsis. Q-S. Trisetum argenteum. Q. Habit. R. Transverse section of leaf blade. S. Floret. (A: Reinecke s. n., JE. B-O: Huter s. n., PR-807305. P: Hellweger et Huter s. n., PR-807301. Q-S: Bornmiüller s. n., B-100526133).

$19^{\circ} 85^{\prime} \mathrm{E}, 15 \mathrm{Jul} 2014$, Barina s. n. E al. (BP); Qafa Valbons, inter vallem Valbona et Theti, $42^{\circ} 24^{\prime} \mathrm{N}, 19^{\circ} 48^{\prime} \mathrm{E}, 4$ Jul 1955, Jávorka s. n. \& Ujhelyi (BP), Beriscus, sub rupe Maja Drosks versus cacumin Maja Hecuravet, $42^{\circ} 23^{\prime} \mathrm{N}$, 1958'E, 1 Jul 1955, Ujhelyi s. n. (BP).
AUSTRIA. Carinthia: Heiligenblut, Heiligenblüter Cavern, $47^{\circ} 2^{\prime} \mathrm{N}$, $12^{\circ} 50^{\prime} \mathrm{E}, 28$ Jul 1906, Jeanpert s. n. (F); Val Pasterze, au-dessus d'Heiligenblut, $47^{\circ} 4^{\prime} \mathrm{N}, 12^{\circ} 46^{\prime} \mathrm{E}$, 26 Jul 1869, Leresche s. $n$. (L); Alpes d’Heiligenblut, Col de Kals, versant nord, $47^{\circ} 2^{\prime} \mathrm{N}, 12^{\circ} 50^{\prime} \mathrm{E}, 31 \mathrm{Jul} 1869$, Leresche s. $n$. (G, L); 


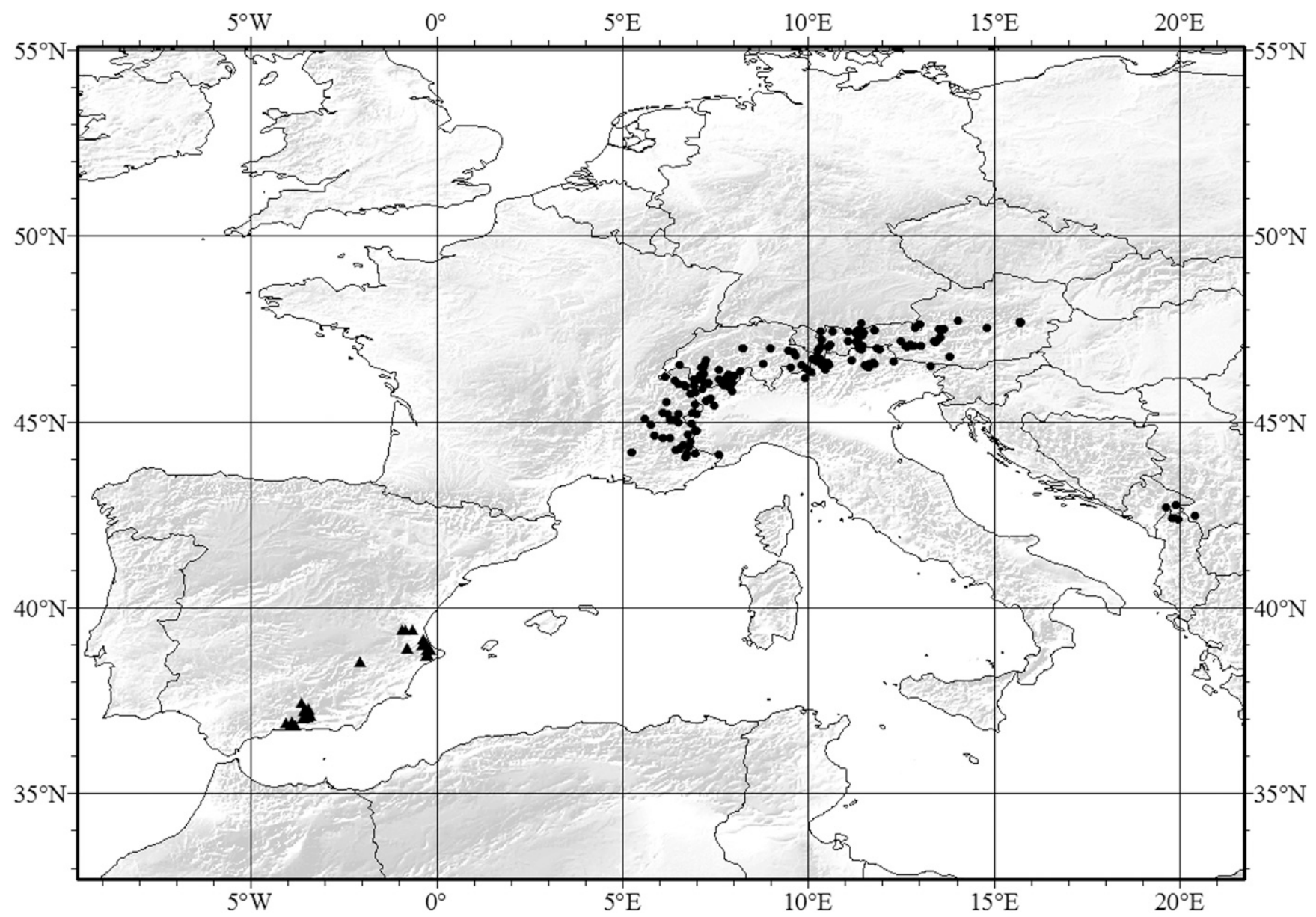

FIG. 3. Distribution of Trisetum velutinum (triangles) and T. distichophyllum (dots).

Heidelberger Hütte, $46^{\circ} 56^{\prime} \mathrm{N}, 10^{\circ} 16^{\prime} \mathrm{E}$, Aug 1952, Raabe s. n. (HBG). Lower Austria: Raxalpe, Preiner Schütt, $47^{\circ} 42^{\prime} \mathrm{N}, 1^{\circ} 43^{\prime} \mathrm{E}$, Jul 1883, Beck s. $n$. (W); Mt. Raxalpe, $47^{\circ} 42^{\prime} \mathrm{N}, 15^{\circ} 43^{\prime} \mathrm{E}$, Aug 1879 , Halácsy s. n. (B, F, JE, PR, WU); Raxalpe, auf der Schütt der Griesleiten, $47^{\circ} 40^{\prime} \mathrm{N}, 15^{\circ} 44^{\prime} \mathrm{E}$, Aug 1871, Halácsy s. n. (PR, W, WU). Salburg: Radstädter Tauern, Lantschfeldgraben im Taurachwinkel, $47^{\circ} 15^{\prime} \mathrm{N}, 1^{\circ} 33^{\prime} \mathrm{E}$, Aug 1916, Vierhapper s. n. (W); Hauptkamm, Höcknerin im Lantschfeldgraben des Taurachwinkels, $47^{\circ} 15^{\prime} \mathrm{N}$, $13^{\circ} 33^{\prime}$ E, Sep 1916, Vierhapper s. n. (W); Dachteingebiet, nächst der Kolpinghütte, $47^{\circ} 29^{\prime} \mathrm{N}, 13^{\circ} 32^{\prime} \mathrm{E}, 17$ Aug 1916, Vetter s. n. (UPS). Styria: Dachstein-Südwand, $47^{\circ} 27^{\prime} \mathrm{N}, 13^{\circ} 36^{\prime} \mathrm{E}$, Aug 1957, Baschant s. n. (MA). Tyrol: Aufstieg vom Hallerangerhaus zum Sunsiger, $47^{\circ} 21^{\prime} \mathrm{N}, 11^{\circ} 28^{\prime} \mathrm{E}$, 28 Aug 1950, Bot. Exk. 740 (HBG); Dorferalpe in Praegrasen, $47^{\circ} 3^{\prime} \mathrm{N}, 12^{\circ} 37^{\prime} \mathrm{E}, 7$ Aug 1865, Gander s. n. (JE, MO); Pettneu, in den Alpen, Weg zur Feuerspitze, $47^{\circ} 12^{\prime} \mathrm{N}, 10^{\circ} 22^{\prime} \mathrm{E}, 17 \mathrm{Jul} 1928$, Gross s. n. (US); vallis Gleirsch, $47^{\circ} 10^{\prime} \mathrm{N}, 11^{\circ} 5^{\prime} \mathrm{E}$, Hellweger s. n. \& Huter (B, C, F, GH, HBG, K, L, NY, O, PR, W, WU); Tyrol sept. ad pedem mt. Tribulaun in Obernberg, $46^{\circ} 59^{\prime} \mathrm{N}, 11^{\circ} 22^{\prime} \mathrm{E}$, Aug 1885 , Huter s. n. (PR); Höttingergraben bei Innsbruck, $47^{\circ} 18^{\prime} \mathrm{N}, 11^{\circ} 22^{\prime} \mathrm{E}, 1868$, Kernu s. n. (WU); Karwendel, $47^{\circ} 25^{\prime} \mathrm{N}, 11^{\circ} 28^{\prime} \mathrm{E}$, Jun 1930, Michaelis s. n. (JE);

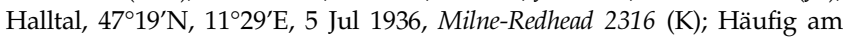
Innsbruck, $47^{\circ} 15^{\prime} \mathrm{N}, 11^{\circ} 23^{\prime} \mathrm{E}$, Jul 1881, Murr s. n. (B); Alpes de Stubai, Vallée de Gschnitz, près du sentier de Gschnitz au Innsbrucker Hütte, au sud de

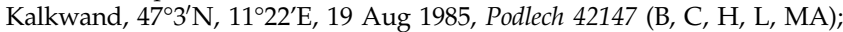
Stubaier Alpen, Fulpmes, Aufstieg Froneben bis Schlicker Alm, $47^{\circ} 9^{\prime} \mathrm{N}$, 11 $18^{\prime}$ E, 26 Aug 1990, Polatschek s. n. (B, C); West Tirol, im Gmeiertal zwischen Pfunds (Oberinntal) und Paznaun, $47^{\circ} 0^{\prime} \mathrm{N}, 10^{\circ} 20^{\prime} \mathrm{E}$, 18 Aug 1906, Handel-Mazzetti s. $n$. (WU); zwischen dem Groder und der Brücke über den Ködnitz Bach, $47^{\circ} 0^{\prime} \mathrm{N}, 12^{\circ} 40^{\prime} \mathrm{E}, 19$ Jul 1905, Handel-Mazzetti s. n. (WU); Nord-Tirol, am Gipfel der Marchreisenspitze in Stubai im Gerölle, $47^{\circ} 10^{\prime} \mathrm{N}$, $11^{\circ} 18^{\prime}$ E, 25 Sep 1902, Handel-Mazzetti s. n. (W); Nörd-Tirol, Sonnwendgebirge bei Jenbach, Spieljoch gegen Osten, $47^{\circ} 28^{\prime} \mathrm{N}, 11^{\circ} 47^{\prime} \mathrm{E}, 18$ Aug 1904, HandelMazzetti s. n. (W); Hohe Tauern, im Kleiner Iseltale bei Trägraten, $47^{\circ} 10^{\prime} \mathrm{N}$, $12^{\circ} 30^{\prime} \mathrm{E}, 7$ Aug 1923 , Vetter s. n. (UPS); Nordtirol, Sonnwendgebirge, $47^{\circ} 28^{\prime} \mathrm{N}$, $11^{\circ} 47^{\prime}$ E, Jul 1905, Vierhapper s. $n$. (W); Nordtirol, Stubaier Alpen, Peilspitze nordwestl, Trins im Gschnitztal, 47 $6^{\prime} \mathrm{N}, 1^{\circ} 23^{\prime} \mathrm{E}, 23$ Aug 1971, Wagenitz 1738 (GOET). Upper Austria: Totes Gebirge, Prielgruppe, Klinserscharte, $47^{\circ} 43^{\prime} \mathrm{N}$, $14^{\circ} 3^{\prime} \mathrm{E}$, 8 Aug 1989, Hörandl 1739 (W); ad pedem meridionalem montis Dachstein, $47^{\circ} 28^{\prime} \mathrm{N}, 13^{\circ} 36^{\prime} \mathrm{E}$, Sep 1905, Hayek s. n. (L, LE, W).

FRANCE. Auvergne-Rhône-Alpes: Isère, Col de l'Arc, près Grenoble, $45^{\circ} 4^{\prime} \mathrm{N}$, 5 ${ }^{\circ} 37^{\prime} \mathrm{E}, 15$ Jun 1884, Beaudouin s. n. (JE); Isère, Mont Chamoux, $45^{\circ} 32^{\prime} \mathrm{N}, 6^{\circ} 12^{\prime} \mathrm{E}$, Jul 1897, Bernard s. n. (HBG); Haute-Savoie, Mt. Vargy,

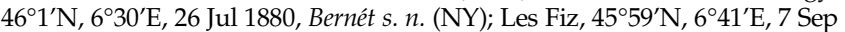
1909, Bouchard s. n. (GB, JE, L, MA, MO, US); Savoie, Maurienne, SaintSorlin-d Arves, $45^{\circ} 13^{\prime} \mathrm{N}, 6^{\circ} 14^{\prime} \mathrm{E}, 31 \mathrm{Jul} 1852$, Didier s. n. (BM, HBG, JE, L, LE, MPU); Savoie, near Col du Mont Cenis, just S of barrage in the Lake, $45^{\circ} 14^{\prime} \mathrm{N}$,

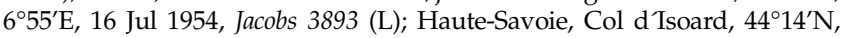
$6^{\circ} 26^{\prime} \mathrm{E}, 11$ Aug 1912, Jeanpert s. n. (F); Isère, Mont Sineipy, près de la Mure, $44^{\circ} 54^{\prime} \mathrm{N}, 5^{\circ} 47^{\prime} \mathrm{E}, 6$ Jul 1890, Moutin 475 \& Bernard (FI, MPU); Haute-Savoie,

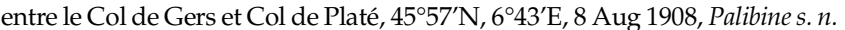
(LE); Savoy, Vallée de Larocheur, en Maurienne, 45 $5^{\circ} 3^{\prime} \mathrm{N}, 6^{\circ} 30^{\prime} \mathrm{E}, 27$ Aug 1856, Perrier de la Bathie s. n. (GH); Savoie, Dauphiné, Le Briançonnais, Serre Chevalier, Rocher Blanc, $45^{\circ} 14^{\prime} \mathrm{N}, 6^{\circ} 6^{\prime} \mathrm{E}, 25$ Jul 1959, Segelberg s. $n$. (S). Provence-Alpes-Côte d'Azur: Hautes-Alpes, Lautaret, $45^{\circ} 2^{\prime} \mathrm{N}, 6^{\circ} 24^{\prime} \mathrm{E}, 16$ Aug 1923, Chandler s. n. (BM); Hautes-Alpes, Ceillac, prés du torrent de Vallon, 44 $40^{\prime} \mathrm{N}, 6^{\circ} 46^{\prime} \mathrm{E}$, 8 Aug 1984, Charpin 19140 (G); Alpes-Maritimes, Saint Martin d'Entraunes, $44^{\circ} 8^{\prime} \mathrm{N}, 6^{\circ} 45^{\prime} \mathrm{E}, 11$ Aug 1982, Charpin AC17607 \& Salanar (G, LE); Basses-Alpes, Barcelonnette, col de Valgelage, $44^{\circ} 23^{\prime} \mathrm{N}$, $6^{\circ} 39^{\prime} \mathrm{E}, 2$ Aug 1897, Coste s. n. (MPU); Hautes-Alpes, Montagne des TroisÉvêchés, au-dessus du village de Goleffe, comm. du Villard-d'Arène, cant.

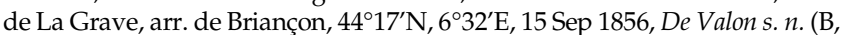
G, GB, GH, L, LE, PR); Hautes-Alpes, Savoie, crete du Galibier, $45^{\circ} 3^{\prime} \mathrm{N}$, 6²4’E, 23 Jul 1955, Dettmann s. n. (B); Hautes-Alpes, Monétier-les-Bains, $44^{\circ} 58^{\prime} \mathrm{N}, 6^{\circ} 30^{\prime} \mathrm{E}$, 22 Aug 1908, Faure s. n. (L); Alpes-Maritimes, Mont Mounier bei Beuil, $44^{\circ} 8^{\prime} \mathrm{N}$, 6 ${ }^{\circ} 58^{\prime} \mathrm{E}, 4$ Aug 1931, Fiedler s. n. (B); Vaucluse, Mont Ventoux, $44^{\circ} 10^{\prime} \mathrm{N}$, $5^{\circ} 16^{\prime} \mathrm{E}, 1852$, Godron s. n. (UPS); Hautes-Alpes, Villar d' Arêne, $45^{\circ} 2^{\prime} \mathrm{N}, 6^{\circ} 20^{\prime} \mathrm{E}, 1851$, Grenier s. n. (UPS); Hautes-Alpes, Briançon, La Combe au Col d'Isoard, $44^{\circ} 14^{\prime} \mathrm{N}, 6^{\circ} 26^{\prime} \mathrm{E}, 1$ Jul 1867, Guillon s. $n$. (UPS); Hautes-Alpes, La Monta près Abriès, Vallon de la Caillante, lac Eugourgeau, $44^{\circ} 46^{\prime} \mathrm{N}, 6^{\circ} 59^{\prime} \mathrm{E}, 19$ Aug 1913, Jeanpert s. n. (F); Hautes-Alpes, La Monta près Abriès, Torrent de Bouchou, $44^{\circ} 46^{\prime} \mathrm{N}, 6^{\circ} 59^{\prime} \mathrm{E}, 18$ Aug 1913, Jeanpert s. n. (F);

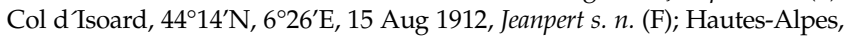


Col du Lautaret, western slopes of Pyramide de Laurichard, $45^{\circ} 2^{\prime} \mathrm{N}, 6^{\circ} 24^{\prime} \mathrm{E}$ 22 Jul 1959, Klimmek s. n. (HBG); Hautes-Alpes, La Grave, $45^{\circ} 2^{\prime} \mathrm{N}, 6^{\circ} 18^{\prime} \mathrm{E}, 10$ Jul 1861, Mathonnet s. n. (JE, LE, MPU, NY, PH); Hautes-Alpes, Gap, Mont Aurouse, $44^{\circ} 38^{\prime} \mathrm{N}$, 5 ${ }^{\circ} 3^{\prime} \mathrm{E}$, 30 Aug 1871, Reverchon s. n. (GH); AlpesMaritimes, Montagne de Rio Freddo, 44 ${ }^{\circ} 7^{\prime} \mathrm{N}, 7^{\circ} 36^{\prime} \mathrm{E}$, $15 \mathrm{Jul}$ 1886, Reverchon s. n. (US); Vaucluse, sommet du Ventoux, $44^{\circ} 10^{\prime} \mathrm{N}, 5^{\circ} 16^{\prime} \mathrm{E}, 12 \mathrm{Jul} 1877$, Reverchon s. n. (JE, MPU); Basses-Alpes, Aurent, près Annot, $44^{\circ} 2^{\prime} \mathrm{N}, 6^{\circ} 42^{\prime} \mathrm{E}$, 7 Jul 1887, Reverchon 255 \& Derbez (MA); Alpes-Maritimes, Mont Mounier be Beuil, Val Demant, $44^{\circ} 8^{\prime} \mathrm{N}, 6^{\circ} 58^{\prime} \mathrm{E}, 4$ Jul 1931, Ronniger s. n. (NY); AlpesMaritimes, Haute-Tinée, Hänge ober Bousiéyas an der Pass-Straße zum Col

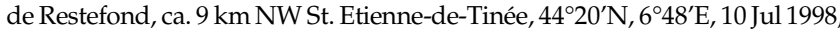
Schneeweiss s. n. \& al. (WU); Hautes-Alpes, am Weg vom Col du Lautaret im oberste Romanche-Tal, $45^{\circ} 2^{\prime} \mathrm{N}, 6^{\circ} 24^{\prime} \mathrm{E}, 14$ Aug 1961, Spanowsky s. n. (JE).

GERMANY. Bavaria: Wimbach Thal, prope Berchtesgaden, $47^{\circ} 37^{\prime} \mathrm{N}$, $13^{\circ} 0^{\prime} \mathrm{E}$, 29 Jul 1858, Ball s. $n$. (US); Mt. Schneeberg, $47^{\circ} 29^{\prime} \mathrm{N}, 13^{\circ} 39^{\prime} \mathrm{E}$, Aug 1884, Beck s. n. (W); Aug 1881, Beck s. n. (WU); Tölz, Benediktenwand, $47^{\circ} 39^{\prime} \mathrm{N}, 11^{\circ} 27^{\prime} \mathrm{E}$, 15 Aug 1894, Bornmüller s. n. (B, JE); Wimbachtal, W. sup. Watzmann, $47^{\circ} 33^{\prime} \mathrm{N}, 12^{\circ} 55^{\prime} \mathrm{E}, 20 \mathrm{Jul} 1915$, Bornmüller s. n. (B); Wetterstein gebirge Schachenplan, $47^{\circ} 25^{\prime} \mathrm{N}, 1^{\circ} 6^{\prime} \mathrm{E}, 14$ Aug 1950, Bot. Exk. 447 (HBG); Berchtesgadener Alpen, Wimbachtal, bei der Kirche, $47^{\circ} 32^{\prime} \mathrm{N}, 12^{\circ} 53^{\prime} \mathrm{E}, 9$ Aug 1963, Lippert s. n. (MA); Wettersteingebirge, westl. Thörlspitze, $47^{\circ} 25^{\prime} \mathrm{N}$, $11^{\circ} 5^{\prime} \mathrm{E}, 14$ Aug 1947, Mäckel s. n. (HBG); Oberstdorf, am Nebelhorn, $47^{\circ} 25^{\prime} \mathrm{N}$ $10^{\circ} 20^{\prime} \mathrm{E}$, Jul 1890, Schmidt s. n. (HBG); Bayerische Alpen, Karwendel, Brunnensteinspitze, $47^{\circ} 24^{\prime} \mathrm{N}, 11^{\circ} 17^{\prime} \mathrm{E}, 24$ Jul 1957, Scholz s. $n$. (B).

ITALY. Aosta Valley: Cogne, Val de Grauson, $45^{\circ} 38^{\prime} \mathrm{N}, 7^{\circ} 23^{\prime} \mathrm{E}$, Aug 1878, Buchenau s. n. (GH); Courmayeur, salendo al Col de Checrouit, $45^{\circ} 47^{\prime} \mathrm{N}, 6^{\circ} 56^{\prime} \mathrm{E}, 20$ Aug 1916, Fiori s. n. (FI); prope Lac de Combal, $45^{\circ} 46^{\prime} \mathrm{N}$, $6^{\circ} 51^{\prime} \mathrm{E}, 11$ Aug 1849, Parlatore s. n. (FI); Gran Paradiso, Gran Lauson nara Cogne, $45^{\circ} 34^{\prime} \mathrm{N}, 7^{\circ} 16^{\prime} \mathrm{E}, 30$ Jun 1965, Strid s. $n$. (S). Friuli-Venecia Julia: Udine province, Kanantal, Pontebba-Chinsaforte, $46^{\circ} 30^{\prime} \mathrm{N}, 13^{\circ} 18^{\prime} \mathrm{E}$, $18 \mathrm{Jul}$ 1913, Ronniger s. n. (BM). Lombardy: Sondrio, Valfurva, pr. St. Catarina, $46^{\circ} 24^{\prime} \mathrm{N}, 10^{\circ} 29^{\prime} \mathrm{E}$, 10 Aug 1870, Ball s. n. (GH, US); Monte Braulio, $46^{\circ} 31^{\prime} \mathrm{N}$, $10^{\circ} 23^{\prime} \mathrm{E}$, 10 Aug 1904, Camperio s. n. (LE); Sondrio, partie inferieure du valle Zebrù, $46^{\circ} 28^{\prime} \mathrm{N}, 10^{\circ} 27^{\prime} \mathrm{E}, 29$ Jul 1887, Cornaz s. n. (MA). Piedmont: Val de Cogne, $45^{\circ} 36^{\prime} \mathrm{N}, 7^{\circ} 21^{\prime} \mathrm{E}, 13$ Aug 1866, Ball s. $n$. (US); environs de $1^{\prime}$ hospice de Valdobbia, $45^{\circ} 49^{\prime} \mathrm{N}, 7^{\circ} 57^{\prime} \mathrm{E}, 26$ Aug 1858, Billot s. $n$. (LE, MPU); Monte Rosa, $45^{\circ} 56^{\prime} \mathrm{N}, 7^{\circ} 52^{\prime} \mathrm{E}, 23$ Jul 1847, Dufft s. n. (JE); Grasjon, 28 Jul 1862, Haussknecht s. n. (JE); M. Chavanisse, 27 Jul 1862, Haussknecht s. n. (JE); Turin, Colle Sestrière, $44^{\circ} 57^{\prime} \mathrm{N}, 6^{\circ} 52^{\prime} \mathrm{E}$, Jul 1864, Rostan s. $n$. (US); Parque Nacional del Gran Paradisso, Valle del Orco, del Gran Piano de Moasca a la Bocheta del Ges, $45^{\circ} 25^{\prime} \mathrm{N}, 7^{\circ} 28^{\prime} \mathrm{E}, 9$ Aug 1952, Sappa s. n. \& Galiano (MA);

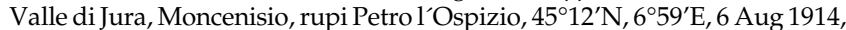
Vignolo-Lutati s. n. (GH). Trentino-Alto Adige/Südtirol: Bolzano, Selva di Val Gardena, Dantercepies, hauteur de Wolkenstein, $46^{\circ} 33^{\prime} \mathrm{N}, 11^{\circ} 45^{\prime} \mathrm{E}, 17$ Aug 1986, Buggenhout s. n. (B, C, H, MA, MPU); Val Gardena, Coronella, Gruppo del Catinaccio, $46^{\circ} 27^{\prime} \mathrm{N}$, $11^{\circ} 37^{\prime} \mathrm{E}, 18$ Aug 1932, Chiarugi s. n. (FI); monte du Stelvio, $46^{\circ} 35^{\prime} \mathrm{N}, 10^{\circ} 32^{\prime} \mathrm{E}, 31 \mathrm{Jul} 1890$, Cornaz s. $n$. (JE, LE); valle Sulden, $46^{\circ} 31^{\prime} \mathrm{N}, 10^{\circ} 35^{\prime} \mathrm{E}, 1884$, Hackel s. n. (US); Brenner, Schelleberg, $46^{\circ} 57^{\prime} \mathrm{N}, 11^{\circ} 26^{\prime} \mathrm{E}$, Aug 1887, Huter s. n. (JE); Sulden, $46^{\circ} 31^{\prime} \mathrm{N}$, $10^{\circ} 35^{\prime} \mathrm{E}$, 9 Jul 1938 , Ronniger s. n. (BM); Schlern, $46^{\circ} 30^{\prime} \mathrm{N}, 11^{\circ} 34^{\prime} \mathrm{E}$, Jul 1890, Schmidt s. $n$. (HBG); Wolferkogl bei Luttach, $46^{\circ} 56^{\prime} \mathrm{N}, 1^{\circ} 55^{\prime} \mathrm{E}, 22$ Aug 1894, Treffer s. $n$. (JE); Riedberg bei Gossensass [Colle Isarco], 46 ${ }^{\circ} 56^{\prime} \mathrm{N}, 11^{\circ} 26^{\prime} \mathrm{E}, 22$ Aug 1904, Handel-Mazzetti s. n. (W); Val Gardena, im Langental in Gröden, $46^{\circ} 34^{\prime} \mathrm{N}$, $11^{\circ} 40^{\prime} \mathrm{E}, 3$ Aug 1903, Vetter s. n. (UPS); Ahrntal, im Grus gegen den Gipfel des Schönberg bei Luttach, $46^{\circ} 58^{\prime} \mathrm{N}, 11^{\circ} 52^{\prime} \mathrm{E}, 29$ Aug 1927, Vierhapper s. $n$. (W). Veneto: Belluno, Drei Zinnen (Tre Cime di Lavaredo), $46^{\circ} 37^{\prime} \mathrm{N}$, $12^{\circ} 18^{\prime} \mathrm{E}, 16$ Aug 1964, Raabe s. $n$. (HBG).

MONTENEGRO. Vasojevići: Kom Vasojevićki, 42²1N, $19^{\circ} 39^{\prime} \mathrm{E}$, Aug 1906, Rohlena s. n. (HBG, PR); montium Ljubonicka planina "dict. Loco Jankova pluga" nominato, $42^{\circ} 45^{\prime} \mathrm{N}, 1^{\circ} 55^{\prime} \mathrm{E}$, Aug 1914, Vandas s. n. (PR). SWITZERLAND. Bern: Rauflihore spitze [Bluttlighore], $46^{\circ} 31^{\prime} \mathrm{N}$, $7^{\circ} 728^{\prime} \mathrm{E}, 12$ Sep 1903, Tranzschel s. n. (LE). Fribourg: versant N des Morteys, $46^{\circ} 34^{\prime} \mathrm{N}, 7^{\circ} 14^{\prime} \mathrm{E}, 3$ Aug 1883, Castella s. $n$. (US). Geneva: Genève, $46^{\circ} 12^{\prime} \mathrm{N}$, $6^{\circ} 9^{\prime} \mathrm{E}, 1839$, Grenier s. $n$. (UPS). Glarus: Glernisch, $46^{\circ} 59^{\prime} \mathrm{N}, 8^{\circ} 59^{\prime} \mathrm{E}, 18 \mathrm{Aug}$ 1846, Dufft s. n. (JE). Grisons: Arosa, beim Arlenwald, $46^{\circ} 47^{\prime} \mathrm{N}, 9^{\circ} 40^{\prime} \mathrm{E}, 8$ Aug 1913, Beger s. n. (B); Puschlav auf dem Sassalbo, $46^{\circ} 20^{\prime} \mathrm{N}, 10^{\circ} 5^{\prime} \mathrm{E}, 20$ Aug 1964, Eckardt 615 (B); Samedan, Piz Padella, 46³1'N, 950’E, 6 Aug 1935, Heimans s. n. \& Jansen (L); Engadin, an der Ofenpass-Strasse unter Ova Spin hinter Zernez, $46^{\circ} 41^{\prime} \mathrm{N}, 10^{\circ} 8^{\prime} \mathrm{E}, 23$ Jul 1936, Koch s. n. (NY); Averstal, Val Bergalga, nördl. Teil südl., Avers-Juppa, $46^{\circ} 27^{\prime} \mathrm{N}, 9^{\circ} 32^{\prime} \mathrm{E}, 26$ Jul 1990, Lewejohann s. n. (GOET); Silvretta, Heildelberg Hütte am Piz Davo Sassé, 46 ${ }^{\circ} 54^{\prime} \mathrm{N}, 10^{\circ} 16^{\prime} \mathrm{E}, 12 \mathrm{Aug}$ 1954, Raabe s. n. (HBG); Calanda über Chur (Coira), 46 54' N , 9²8'E, Sep 1917, Schröter s. n. \& Braun-Blanquet (C, GH, O, PR, W); Alp Sesvenna und Scarl, 46 $42^{\prime}$ N , 10 $20^{\circ}$ E, 10 Aug 1949, Simon s. $n$. (GB); Passhöhe des Ofenpasses, $46^{\circ} 38^{\prime} \mathrm{N}, 10^{\circ} 17^{\prime} \mathrm{E}, 29 \mathrm{Jul} 1966$, Simon s. n. (C, $\mathrm{H})$; Chur, Gromserkopf [Gromser Chopf], près de la Fürstenalp, près de
Coire, 46 $52^{\prime} \mathrm{N}$, 9 ${ }^{\circ} 36^{\prime} \mathrm{E}, 15$ Aug 1888, Stebler 114 \& Schröter (C, LE, NY); Graubunden, Zerner, M. Buffalora, 46 $37^{\prime} \mathrm{N}, 10^{\circ} 15^{\prime} \mathrm{E}$, $27 \mathrm{Jul} 1923$, Thaysén s. n. (H); Lango de Spoor bij Bernina-häuser, $46^{\circ} 24^{\prime} \mathrm{N}, 10^{\circ} 0^{\prime} \mathrm{E}$, Jul 1929, Soest s. n. (L); Val Plavna, $46^{\circ} 42^{\prime} \mathrm{N}, 10^{\circ} 13^{\prime} \mathrm{E}$, Jul 1932, Jansen s. n. (L). Lucerne: Mt. Pilato, $46^{\circ} 58^{\prime} \mathrm{N}, 8^{\circ} 15^{\prime} \mathrm{E}$, Baching s. $n$. (HBG); Obwalden, Pilatus vid Ringfluhe, $46^{\circ} 58^{\prime} \mathrm{N}, 8^{\circ} 15^{\prime} \mathrm{E}, 20$ Sep 1812 , Wahlenberg s. $n$. (UPS). Ticino: Lukmanierpass, Campo Solario, 46 $33^{\prime} \mathrm{N}, 8^{\circ} 48^{\prime} \mathrm{E}, 20 \mathrm{Jul}$ 1912, Beger s. n. (B). Tyrol: in alpibus Leontinis, 8 Aug 1954, Duty s. $n$. (JE). Valais: convalle alpina Nicolai Thal prope Zermatt, $46^{\circ} 11^{\prime} \mathrm{N}, 7^{\circ} 48^{\prime} \mathrm{E}$, Aug 1845 , Ball s. $n$.

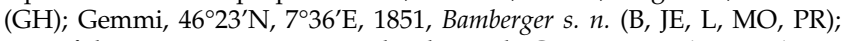
Massif du Mont Rose, moraines du glacier de Gorner, $45^{\circ} 58^{\prime} \mathrm{N}, 7^{\circ} 48^{\prime} \mathrm{E}, 31$ Aug 1916, Beauverd s. n. (NY); supra Zinal, $46^{\circ} 8^{\prime} \mathrm{N}, 7^{\circ} 37^{\prime} \mathrm{E}, 13$ Aug 1887, Bernoulli s. n. (MA, MPU); Saastal, Wege nach Plattjen, $46^{\circ} 6^{\prime} \mathrm{N}, 7^{\circ} 55^{\prime} \mathrm{E}, 15 \mathrm{Jul}$ 1945, Bührer s. n. (UPS); L'Erié, Grand Chavalard, chemin du Lac de Fully, $46^{\circ} 10^{\prime} \mathrm{N}, 7^{\circ} 6^{\prime} \mathrm{E}, 13 \mathrm{Jul}$ 1990, Castroviejo 11213 SC \& al. (MA); Zermatt, Schwarzsee, $46^{\circ} 39^{\prime} \mathrm{N}, 7^{\circ} 16^{\prime} \mathrm{E}, 11$ Aug 1894, Clarke 48718 (K); Zermatt, moraene van de Findelenglesscher, $46^{\circ} 6^{\prime} \mathrm{N}, 7^{\circ} 51^{\prime} \mathrm{E}$, 29 Jul 1922, Danser 5050 (L); Val des Dix, Pas du Chat, extrémité sud du Lac des Dix, $46^{\circ} 27^{\prime} \mathrm{N}$, $7^{\circ} 12^{\prime} \mathrm{E}, 1$ Aug 1971, Dutoit s. $n$. (HBG, UPS); Verbier, $46^{\circ} 5^{\prime} \mathrm{N}, 7^{\circ} 12^{\prime} \mathrm{E}, 10 \mathrm{Aug}$ 1968, Eckardt 1056 (B); Mattmark, 462'N, 757'E, 1901, Goethart s. n. \& Jongmans (L); Gornergletscherns ändmorän, $46^{\circ} 0^{\prime} \mathrm{N}, 7^{\circ} 44^{\prime} \mathrm{E}, 27 \mathrm{Jul} 1883$, Huld s. n. (H); Zermatt, moraine van de Findelenglesscher, $46^{\circ} 6^{\prime} \mathrm{N}, 7^{\circ} 51^{\prime} \mathrm{E}$, Aug 1937, Jansen s. $n$. (L); in valle Binn, $46^{\circ} 21^{\prime} \mathrm{N}, 8^{\circ} 11^{\prime} \mathrm{E}$, Lagger s. $n$. (UPS); Westseite des Trifthorn östl. Saas-Fee, $46^{\circ} 6^{\prime} \mathrm{N}, 7^{\circ} 55^{\prime} \mathrm{E}, 13 \mathrm{Jul}$ 1981, Lewejohann s. n. (GOET); Gornergletscher, $45^{\circ} 58^{\prime} \mathrm{N}, 7^{\circ} 49^{\prime} \mathrm{E}$, Aug 1894 , Linder s. n. (MA); Saasthal, 46 ${ }^{\circ} 8^{\prime} \mathrm{N}, 7^{\circ} 56^{\prime} \mathrm{E}, 15$ Aug 1872, Morthier s. n. (JE); Saas-Fee und der Gletscheralpen, $46^{\circ} 6^{\prime} \mathrm{N}, 7^{\circ} 55^{\prime} \mathrm{E}$, 28 Jul 1910, Poeverlein s. n. (LE); Simplon, an der Strasse nördlich der Passhöhe, 46 $15^{\prime} \mathrm{N}, 8^{\circ} 1^{\prime} \mathrm{E}$, 28 Jul 1906, HandelMazzetti s. n. (WU); Portail de Fully, $46^{\circ} 9^{\prime} \mathrm{N}, 7^{\circ} 5^{\prime} \mathrm{E}, 21 \mathrm{Jul} 1874$, Vigener s. n. (B); Zermatt, über dem Riffelberg, $45^{\circ} 59^{\prime} \mathrm{N}, 7^{\circ} 45^{\prime} \mathrm{E}$, Aug 1901, Wolfs. $n$. (B, C, GH, JE, L, LE, MA, O, PR, S, US, W). Vaud: Anzeindaz, Alpes de Bex, eboulis de Diablerets, $46^{\circ} 18^{\prime} \mathrm{N}, 7^{\circ} 11^{\prime} \mathrm{E}, 10$ Aug 1880, Chenevard s. $n$. (JE); Alpes de Châteaux-d'Oex, à la Hausseresse et sous le rocher des Tours, $46^{\circ} 28^{\prime} \mathrm{N}, 7^{\circ} 7^{\prime} \mathrm{E}$, Leresche s. $n$. (F, K, JE).

Notes-Jonsell (1980), who probably followed Paunero (1950), cited Trisetum distichophyllum from the eastern Pyrenees. Unfortunately, this record corresponds to material collected by Rivas Mateos, the labelling of which is questioned by many authors (i.e. Rothmaler 1935, Benedí and Sáez 1996, Bolòs and Vigo 2001). After the study of the herbarium material of this species, we found that the westernmost populations of T. distichophyllum are found in the French Alps.

Jávorka (1919) described a new species from the mountains of northern Albania, T. albanicum Jávorka, separating it from T. distichophyllum because of its flat, less rigid, and green leaves with less marked nerves, less puberulous surfaces, and margins always with hairy to ciliate, more contracted panicles, and shorter callus hairs. Later, Hayek (1932) combined it as a subspecies of T. distichophyllum and Rohlena (1942) published a new record of the subspecies from eastern Montenegro. We consider this subspecies a synonym of $T$. distichophyllum, not finding significant differences from the rest of the material to justify separation. The specimens studied from the Balkans have greyish to greenish leaves, flat to conduplicate basal and top leaf blades, and variable indumentum. Comparing the shape of the panicles, we have observed that it is quite variable in the specimens from Albania and the Alps, while the length of the callus indumentum in the Alps specimens is similar to that in the Albanian ones ((1.5-)2.2-3.7(-4.5) $\mathrm{mm}$ and 2.5-3 mm length, respectively).

Beauverd (1934) described a new subspecies from the French Alps, T. distichophyllum subsp. delphinense Beauverd, characterized by its apically and irregularly torn-toothed ligules with ciliate margins, lower glumes with one or two lateral rudimentary nerves, and upper glumes with an acuminate or muticous apex. We consider this subspecies a synonym of T. distichophyllum. The material of the species we studied is highly variable, having ligules $0.5-1 \mathrm{~mm}$ long, with a laciniate to slightly laciniate apex, rarely slightly lobulated, lower glumes with a variable number of nerves, and upper glumes 
with an acuminate to long acuminate apex, sometimes acute. Beauverd also differentiated this subspecies by the inrolled or conduplicate leaves, contrasting it with $T$. argenteum, but these characters are also present in T. distichophyllum.

2. Trisetum argenteum (Willd.) Roem. \& Schult., Syst. Veg. 2: 665. 1817. Avena argentea Willd., Enum. Pl.: 125. 1809. Trisetum distichophyllum var. argenteum (Willd.) Ducommun, Taschenb. Schweiz. Bot.: 858. 1869. Trisetaria argentea (Willd.) Banfi \& Soldano, Atti Soc. Ital. Sci. Nat. Mus. Civico Storia Nat. Milano 135: 382. 1996.-TYPE: [unknown locality and collector] (lectotype, here designated, B-W-02187-020 (image!)).

T. argenteum var. parviflorum Parl., Fl. Ital. 1: 264. 1850.-TYPE: ITALY. Bergamo, Monte Resegone, $45^{\circ} 51^{\prime} \mathrm{N}, 9^{\circ} 28^{\prime} \mathrm{E}, 1844$, L. Rota s. n. (lectotype, here designated, FI!; isolectotype, BERG-31548 (image!)).

Herbs (14.7-)23.3-35.4(-36.6) cm tall, short rhizomatous, loosely tufted, culms (0.2-)0.3-0.5 mm diam, glabrous; nodes (3-)4-5(-6), concentrated in lower part of the culm, glabrous. Basal leaf sheaths puberulous, with hairs up to $0.2 \mathrm{~mm}$ long, decaying into fibers, brownish to yellowish, sometimes greenish; basal leaf blades (1.2-)2.4-3.8(-6.7) $\mathrm{cm}$ long $\times$ (0.9-)1.2-1.5(-1.7) mm wide, flat to inrolled, pubescent to slightly puberulous adaxially and abaxially, sometimes glabrous, with hairs (0.05-)0.1(-0.8) mm long, greyish to greenish, sometimes yellowish to brownish; top culm leaf sheaths (4-) 6-8.7(-9) cm long, shorter than the internodes, sometimes longer or almost equal, glabrous, without cilia, or sometimes with; top culm leaf blades (1.7-)2.2-3.5(-6) cm long $\times 0.9-1.5$ $(-1.8) \mathrm{mm}$ wide, flat to conduplicate, rarely parallel to the culm, glabrous to pubescent adaxially, puberulous to slightly puberulous abaxially, sometimes with short hairs on the margins up to $0.1 \mathrm{~mm}$ long, greenish to greyish; inner collar region glabrous, with scattered hairs or slightly puberulous on the margins, with hairs (0.05-)0.2-0.8(-1) mm long; ligules $0.3-0.5(-0.7) \mathrm{mm}$ long, pubescent, rarely glabrous or with some scattered hairs on the surface, apex laciniate with hairs up to $0.15 \mathrm{~mm}$ long. Basal node of the panicle glabrous. Panicles (3.7-)5.4-7(-7.6) $\mathrm{cm}$ long $\times(0.9-) 1.2-2.3(-3.2) \mathrm{cm}$ wide, oblong to elliptic in outline, sometimes narrowly elliptic to lanceolate, lax; rachis glabrous, rarely slightly puberulous or pubescent, with hairs up to $0.08 \mathrm{~mm}$ long; longest basal branches (0.4-)0.8-1.3(-2.2) $\mathrm{cm}$. Spikelets (4.8-)5.6-6.5(-8) $\mathrm{mm}$ long $\times(1.4-) 2-4(-5.7) \mathrm{mm}$ wide, (1-)2-3-flowered; pedicels (2.2-)3.2-4.2(-5.4) $\mathrm{mm}$, glabrous to slightly pubescent, with hairs up to $0.1 \mathrm{~mm}$ long. Glumes unequal (ratio lower glume length/upper glume length $=(0.59-) 0.65-0.78(-0.87))$; lower glume (2.7-)3.5-4.3 $(-5.7) \mathrm{mm}$ long $\times 0.5-0.8(-1.4) \mathrm{mm}$ wide, narrowly to broadly lanceolate, sometimes narrowly elliptic or linear (ratio lower glume width/lower glume length $=(0.14-) 0.16-0.21(-0.25)$, acuminate, sometimes acute, 1-nerved, glabrous, sometimes with short hairs up to $0.1 \mathrm{~mm}$ long from the center to the upper part of the nerve, greenish to yellowish, sometimes surrounded by purple on the laterals and upper part; upper glume (4.6-)5-6(-7.3) mm long $\times(1.2-) 1.4-1.6(-2) \mathrm{mm}$ wide, narrowly elliptic or elliptic to narrowly lanceolate or oblong, rarely oblanceolate (ratio upper glume width/upper glume length $=(0.22-) 0.24-0.3)$, acuminate to long acuminate, rarely acute, 3-nerved, glabrous, with short hairs up to $0.08 \mathrm{~mm}$ long from the middle to the upper part of the central nerve, greenish to yellowish on the central part, usually surrounded by purple; rachilla segment between first and second floret (0.6-)1.1-1.3(-1.4) mm long, with hairs (1-)1.7-2.5(-3.4) mm long; rachilla segments to sterile floret 1.3-1.7(-2) $\mathrm{mm}$ long, with hairs (0.8-)1.2-1.6(-2) mm long. Lemmas (4-)4.4-5.2(-6.5) $\mathrm{mm}$ long $\times 0.5-0.8(-1.4) \mathrm{mm}$ wide, broadly lanceolate to oblong or elliptic, rarely narrowly lanceolate (ratio lemma width/lemma length $=(0.19-) 0.25-0.33)$, glabrous to scabridulous, with hairs up to $0.1 \mathrm{~mm}$ long on the central and upper part, greenish on the central part, sometimes yellowish, usually surrounded by purple; apical teeth without or $0.1-0.3(-0.4) \mathrm{mm}$ long, with aristules (0.1-)0.3-0.5(-0.7) mm long; awn (3.7-)5.2-6.4(-7.2) $\mathrm{mm}$ long, inserted (1.6-)1.8-2.4(-3.2) $\mathrm{mm}$ from the base (ratio awn insertion from the base length/lemma length $=(0.35-) 0.42-0.46(-0.5))$, geniculate and slightly twisted near the base, with very short adpressed hairs up to $0.1 \mathrm{~mm}$ long; callus (0.2-) $0.3 \mathrm{~mm}$ long, oblong to elliptic, with hairs (0.7-)1.4-1.8(-2.6) mm long. Palea (3.5-)3.8-4.2(-5.8) $\mathrm{mm}$ long $\times(0.9-) 1-1.3(-1.4) \mathrm{mm}$ wide (ratio palea length/lemma length $=(0.73-) 0.79-0.89(-0.98))$, narrowly elliptic to elliptic, glabrous or with hairs from the middle to the upper part of the outer edges; keels with short antrorse hairs mostly on the upper part, rarely glabrous, ending in teeth $0.1-0.3(-0.4) \mathrm{mm}$ long. Lodicules $(0.4-) 0.5-0.8(-0.9) \mathrm{mm}$ long, with apex shortly and irregularly lobate. Anthers (1.5-)1.9-2.3(-2.4) mm long. Ovary (0.4-)0.6-0.8(-2.3) $\mathrm{mm}$ long, glabrous. Caryopsis $2-2.7 \mathrm{~mm}$ long $\times 0.4-0.7 \mathrm{~mm}$ wide. Figure 2 .

Phenology-Flowering from June to September.

Chromosome Number-2n = 28 (Beuret 1974; Lippert 2006).

Distribution and Habitat-Trisetum argenteum occurs in the eastern Alps, from Austria and Italy, reaching to Slovenia; at 200-2,330 m elevation; around rocks and pebbles, in calcareous soils. Figure 5.

Specimens Examined-AUSTRIA. Carinthia: Loiblpass, Tschanko-Fall, $46^{\circ} 26^{\prime} \mathrm{N}, 14^{\circ} 16^{\prime} \mathrm{E}, 12 \mathrm{Jul} 1954$, Lohmar s. n. (HBG); in valle Bartolograben, prope Saifnitz, $46^{\circ} 34^{\prime} \mathrm{N}, 1^{\circ} 34^{\prime} \mathrm{E}, 24 \mathrm{Jul} 1914$, Aust s. n. (GB); Volaia, $46^{\circ} 37^{\prime} \mathrm{N}, 12^{\circ} 53^{\prime} \mathrm{E}, 21$ Sep 1857, Ball s. n. (K, US); Polinik Gipfel, $46^{\circ} 37^{\prime} \mathrm{N}$, $12^{\circ} 58^{\prime} \mathrm{E}, 12$ Aug 1952, Doppelbaur s. n. (M); in glareosis vallis Loibel [Loibl], $46^{\circ} 26^{\prime} \mathrm{N}, 14^{\circ} 16^{\prime} \mathrm{E}$, Aug 1883, Jabornegg s. n. (B, BM, C, F, FI, MO, NY, PR, W, WU); Ostkarawanken, Petzen, am Hauptweg zw. Berg- u. Talstation der

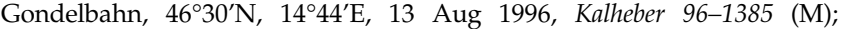
Karawanken, Hochobir-Süd, Auffahrt zur Eisenkappler Hütte, Seitentälchen unterh, Berghaus Fladung, 46 $29^{\prime} \mathrm{N}, 14^{\circ} 31^{\prime} \mathrm{E}, 20$ Aug 1991, Leute s. n. \& Kosch (WU); Carinthia, $46^{\circ} 45^{\prime} \mathrm{N}, 13^{\circ} 49^{\prime} \mathrm{E}$, Aug 1878, Marchesetti s. $n$. (W); Karawanken, Zell-Pfarre, am Hochobir, 46 $34^{\prime} \mathrm{N}, 13^{\circ} 34^{\prime} \mathrm{E}, 11$ Aug 1949 , Merxmüller 5533 \& Wiedmann (M); Sanntaler Alpen, Sulzbach, 47 $41^{\prime} \mathrm{N}$, $13^{\circ} 37^{\prime}$ E, 2 Sep 1904, Hayek s. n. (GB); Karnische Alpen, Sittmoosergraben bei Mauthen, $46^{\circ} 41^{\prime} \mathrm{N}, 12^{\circ} 57^{\prime} \mathrm{E}, 30$ Aug 1926, Vierhapper s. n. (W); Petzen-Alp, ober Ritschberg, $46^{\circ} 30^{\prime} \mathrm{N}, 14^{\circ} 44^{\prime} \mathrm{E}$, Welden s. n. \& Freyer (B, JE, UPS, WU); Karawanken, bei Feistritz im Rosental nahe der Kirche, $46^{\circ} 31^{\prime} \mathrm{N}, 14^{\circ} 10^{\prime} \mathrm{E}, 16$ Jul 1956, Wagenitz 1738 (GOET). Lower Austria: Raxer Alpen, 47² $42^{\prime} \mathrm{N}$, $15^{\circ} 43^{\prime} \mathrm{E}$, Hausmann s. $n$. (B). Styria: Sulzbach, $46^{\circ} 48^{\prime} \mathrm{N}, 15^{\circ} 53^{\prime} \mathrm{E}$, Unger s. $n$. (M); prope refugium alpinum "Okreselhütte" in alpibus Lithopolitanis (Steiner-sive Sanntaler Alpen), 46 $22^{\prime} \mathrm{N}, 14^{\circ} 34^{\prime} \mathrm{E}$, Aug 1904, Hayek s. n. (BM, GB, H, L, O, W). Tyrol: juxta Lienz, $46^{\circ} 49^{\prime} \mathrm{N}, 12^{\circ} 46^{\prime} \mathrm{E}$, 9 Sep 1854 , Ball s. $n$. (F, US); pr. Lienz, Kerschbaumeralpe, $46^{\circ} 49^{\prime} \mathrm{N}, 12^{\circ} 46^{\prime} \mathrm{E}$, 19 Aug 1872, Gander s. n. (JE); Sep 1855, Molendo sn. (M); ober Tres im Nonsberg an steinigen Stellen, $47^{\circ} 17^{\prime} \mathrm{N}, 1^{\circ} 42^{\prime} \mathrm{E}, 26$ Aug 1902, Handel-Mazzetti s. n. (W); Kerschbaumer bei Lienz, $46^{\circ} 46^{\prime} \mathrm{N}, 12^{\circ} 46^{\prime} \mathrm{E}, 30 \mathrm{Jul} 1883$, Witting s. $n$. (B).

ITALY: Friuli-Venecia Julia: Provincia di Udine, prope Forno Avoltri, $46^{\circ} 35^{\prime} \mathrm{N}, 12^{\circ} 46^{\prime} \mathrm{E}$, Sep 1857 , Ball s. $n$. (MO); Udine province, vallée de la Cimoliana [Cimolais], Rifugio Pordenone, $46^{\circ} 22^{\prime} \mathrm{N}, 1^{\circ} 29^{\prime} \mathrm{E}, 7$ Aug 1979, Charpin 15786 (GOET, MA); Prov. Udine, Alpi Carniche, ander Strasse Misurina-Tolmezzo, im Tal des Tagliamento bei Forno di Sotto, $46^{\circ} 23^{\prime} \mathrm{N}$, 1240’E, 16 Jul 1992, Lippert 26158 (M); Prov. Pordenone, Prealpi Carniche, Spalti di Toro range N Cimolais, $46^{\circ} 15^{\prime} \mathrm{N}, 12^{\circ} 28^{\prime} \mathrm{E}$, 11 Sep 2012, Müller 10983 (JE); Raibl-Mangart, $46^{\circ} 26^{\prime} \mathrm{N}, 13^{\circ} 39^{\prime} \mathrm{E}$, Vierhapper s. n. (W). Lombardy: monte Campione prope Lecco, $45^{\circ} 51^{\prime} \mathrm{N}, 9^{\circ} 23^{\prime} \mathrm{E}$, 8 Sep 1857, Ball s. $n$. (US); Lecco province, montis Grigna, versus Lacum Larium [Como Lake], $45^{\circ} 57^{\prime} \mathrm{N}$, 9 $9^{\circ} 23^{\prime} \mathrm{E}$, 15 Sep 1855, Ball s. $n$. (US); Como province, Grigna meridionale, Canale Porta, $45^{\circ} 55^{\prime} \mathrm{N}, 9^{\circ} 23^{\prime} \mathrm{E}$, 17 Jul 1950, Berger s. n. (M);

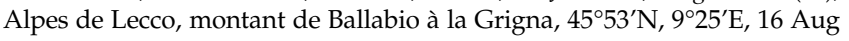


1859, Leresche s. n. (JE, L); Mt. Braulio, $46^{\circ} 31^{\prime} \mathrm{N}, 10^{\circ} 23^{\prime} \mathrm{E}, 3$ Aug 1902, Longe s. n. (MPU); Como, Grigna Meridionale, "Piana delle Groppe", $45^{\circ} 56^{\prime} \mathrm{N}$ 9²3’E, 27 Jul 1965, Simon s. n. (HBG, S). Trentino-Alto Adige/Südtirol: Grigna Meridionale, Cresta Segantini, 46² $12^{\prime} \mathrm{N}, 1^{\circ} 42^{\prime} \mathrm{E}, 12$ Aug 1952 , Reznik s. n. (M); Pusteria, Landro, $46^{\circ} 37^{\prime} \mathrm{N}, 12^{\circ} 13^{\prime} \mathrm{E}$, Auperdorfer s. n. (PR). Montis Schlehren supra Ratzes, $46^{\circ} 30^{\prime} \mathrm{N}, 1^{\circ} 34^{\prime} \mathrm{E}, 23$ Aug 1869, Ball s. $n$ (US); Val di Fassa, $46^{\circ} 26^{\prime} \mathrm{N}, 11^{\circ} 42^{\prime} \mathrm{E}, 27$ Aug 1860, Ball s. $n$. (US); supra Val Rendena, montis Brenta, $46^{\circ} 10^{\prime} \mathrm{N}, 10^{\circ} 53^{\prime} \mathrm{E}$, 4 Sep 1858 , Ball s. $n$. (GH); supra Val Selva, prope jugum Crostè [Cima Groste], 46 $11^{\circ} \mathrm{N}, 10^{\circ} 54^{\prime} \mathrm{E}$, Aug 1875 Ball s. n. (US); Trento prov., in sinu Val Selva prope Malè, $46^{\circ} 21^{\prime} \mathrm{N}, 10^{\circ} 54^{\prime} \mathrm{E}$ 18 Aug 1860, Ball s. n. (US); Seiseralp, 46 ${ }^{\circ} 31^{\prime} \mathrm{N}, 1^{\circ} 32^{\prime} \mathrm{E}, 24$ Aug 1860, Ball s. n. (F, US); Trento province, in monte Scanuppia, $45^{\circ} 57^{\prime} \mathrm{N}, 11^{\circ} 9^{\prime} \mathrm{E}, 11$ Aug 1860, Ball s. n. (MO, US); Giudicaria, Monte Tombea, $45^{\circ} 48^{\prime} \mathrm{N}, 10^{\circ} 37^{\prime} \mathrm{E}$, Jul 1952, Baschant s. n. (B, JE, MA); Val dei Concei (W. van het Gardameer), $45^{\circ} 54^{\prime} \mathrm{N}, 10^{\circ} 43^{\prime} \mathrm{E}, 9$ Aug 1959, Boom s. n. (L); Monte Cristallo, $46^{\circ} 34^{\prime} \mathrm{N}$ $12^{\circ} 11^{\prime} \mathrm{E}$, 23 Aug 1896, Bornmüller s. n. (B, JE); in rupestribus vallis Prags, $46^{\circ} 43^{\prime} \mathrm{N}, 12^{\circ} 7^{\prime} \mathrm{E}, 20$ Aug 1896, Bornmüller s. n. (B, BM, C, F, FI, K, L, MO, NY, PR, S, W, WU); Bolzano, Pustaria, Geierwände bei Schenderbach-Landro, $46^{\circ} 37^{\prime} \mathrm{N}, 12^{\circ} 13^{\prime} \mathrm{E}, 20$ Aug 1896, Bornmüller s. n. (JE); Monte Cristallo, Val Fonda, $46^{\circ} 34^{\prime} \mathrm{N}, 12^{\circ} 11^{\prime} \mathrm{E}, 23$ Aug 1896, Bornmüller s. n. (B); Monte Bondone, $45^{\circ} 59^{\prime} \mathrm{N}, 11^{\circ} 1^{\prime} \mathrm{E}$, De Sardagna s. n. (W); Bocca di Brenta, 9 Aug 1884, De Sardagna s. n. (W); Bolzano province, Tiers östlich Bozen, Südseite des Tschafon, 46 $28^{\circ} \mathrm{N}, 1^{\circ} 31^{\prime} \mathrm{E}, 21$ Jul 1984, Dersch 4488 (GOET); Bozen, Daumkofel (Prags), 0.6 km SSE Daumkofel, $12^{\circ} 8^{\prime} \mathrm{N}, 46^{\circ} 41^{\prime} \mathrm{E}, 26$ Jul 2003, Hilpold s. n. \& Kiebacher (W); Pusteria, Landro, 46 $37^{\circ}$ N , 12 ${ }^{\circ} 13^{\prime} \mathrm{E}, 27 \mathrm{Jul} 1869$ Huter 1171 (B, MPU); Bolzano, Schlern, 46 $30^{\prime} \mathrm{N}, 1^{\circ} 34^{\prime} \mathrm{E}, 15 \mathrm{Jul} 1967$, Huter s. n. (B); Pusteria, valle Virchlein, Sexten, $46^{\circ} 42^{\prime} \mathrm{N}, 12^{\circ} 20^{\prime} \mathrm{E}, 23$ Aug 1878, Huter s. n. (GB); Bad Ratzes, $46^{\circ} 30^{\prime} \mathrm{N}, 11^{\circ} 34^{\prime} \mathrm{E}, 3$ Aug 1893 , Jaap s. n. (HBG); Val d'Adige [Etschtal], Salurn, $46^{\circ} 14^{\prime} \mathrm{N}, 1^{\circ} 12^{\prime} \mathrm{E}$, 2 Jun 1952, Merxmüller 5534 \& Wiedmann (M); Fassatal valley, Moena, $46^{\circ} 22^{\prime} \mathrm{N}, 11^{\circ} 39^{\prime} \mathrm{E}$, Jul 1912, Paul s. n. (L); vallis di Bono, $46^{\circ} 1^{\prime} \mathrm{N}, 10^{\circ} 50^{\prime} \mathrm{E}$, Jul 1889, Porta s. $n$. (JE); Ledro, $45^{\circ} 53^{\prime} \mathrm{N}$, 1044'E, 2 Aug 1883, Porta s. n. (B, G, MA, WU); Val di Ledro, $45^{\circ} 53^{\prime} \mathrm{N}, 10^{\circ} 44^{\prime} \mathrm{E}, 20 \mathrm{Jul} 1881$, Porta s. n. (JE); vallis di Bono, $46^{\circ} 1^{\prime} \mathrm{N}, 10^{\circ} 50^{\prime} \mathrm{E}$ Jul 1892, Porta s. n. (M); circa pagum Cologna, $45^{\circ} 55^{\prime} \mathrm{N}, 10^{\circ} 38^{\prime} \mathrm{E}$, Jul 1896, Porta s. n. (MPU); San Martino di Castrozza, $46^{\circ} 16^{\prime} \mathrm{N}, 11^{\circ} 48^{\prime} \mathrm{E}, 5$ Aug 1895 , Saint-Lager s. n. (G, L, NY); pr. Salurn, $46^{\circ} 14^{\prime} \mathrm{N}, 11^{\circ} 12^{\prime} \mathrm{E}$, Sauter s. $n$. (W) Ratzes, $46^{\circ} 30^{\prime} \mathrm{N}, 11^{\circ} 34^{\prime} \mathrm{E}, 13 \mathrm{Jul} 1903$, Schultz s. n. (B); Bade Ratzes, $46^{\circ} 30^{\prime} \mathrm{N}$,

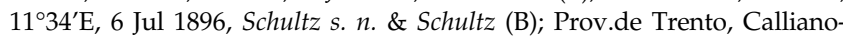
Folgaria, 45⒌' $\mathrm{N}, 11^{\circ} 10^{\prime} \mathrm{E}, 19$ Aug 1960, Segelberg s. n. (S); Trento province,

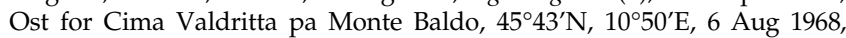
Thornberg s. n. (C); Bolzano, Wolkenstein (Selva di Val Gardena), Grödnertal (Val Gardena), à gauche du Rio Gardena, 46 $33^{\prime} \mathrm{N}, 1^{\circ} 45^{\prime} \mathrm{E}, 7$ Aug 1985, Buggenhout s. n. (B, C, H, L, M, MA); Bolzano (Bozen), am Wege von St. Constantin nach Hanenstein, im Gerölle, 46 $32^{\prime} \mathrm{N}, 1^{\circ} 31^{\prime} \mathrm{E}, 12$ Jul 1905 , Handel-Mazzetti s. n. (GB, WU); monte Schlern, $46^{\circ} 30^{\prime} \mathrm{N}, 11^{\circ} 34^{\prime} \mathrm{E}, 4$ Aug 1884 Vatke s. n. (JE); Val Gardena, im Langental in Gröden bei Wolkenstein, 46 $34^{\prime} \mathrm{N}, 11^{\circ} 40^{\prime} \mathrm{E}, 12$ Aug 1907, Vetter s. n. (UPS); Saint Ulrich in Gröden [Ortisei], 46 $34^{\prime} \mathrm{N}, 1^{\circ} 40^{\prime} \mathrm{E}, 20$ Aug 1913, Zinsmeister s. n. (M); Seiser Alp $46^{\circ} 31^{\prime} \mathrm{N}, 11^{\circ} 32^{\prime} \mathrm{E}$, Jul 1828, Zuccarini s. n. (M). Veneto: Monte Pasubio, $45^{\circ} 47^{\prime} \mathrm{N}$, $11^{\circ} 10^{\prime} \mathrm{E}$, Ball s. n. (NY); Val de Signori, prope Schio, $45^{\circ} 42^{\prime} \mathrm{N}, 11^{\circ} 21^{\prime} \mathrm{E}, 27 \mathrm{Aug}$ 1858 , Ball s. $n$. (US); in verticibus Pasubio et Covelalto, $45^{\circ} 47^{\prime} \mathrm{N}, 11^{\circ} 10^{\prime} \mathrm{E}, 27$ Aug 1858 , Ball s. $n$. (US); Vicenza province, near Recoaro, $45^{\circ} 41^{\prime} \mathrm{N}, 11^{\circ} 13^{\prime} \mathrm{E}$, Jun 1863, Ball s. n. (GH, NY); Cortina d'Ampezzo, in Monte Tofana, $46^{\circ} 32^{\prime} \mathrm{N}$, $12^{\circ} 3^{\prime} \mathrm{E}, 30$ Aug 1870, Ball s. n. (US); Val di Portole, Sette Comuni, $45^{\circ} 54^{\prime} \mathrm{N}$ $11^{\circ} 30^{\prime} \mathrm{E}$, Sep 1860 , Ball s. n. (US); Lago di Alleghe, $46^{\circ} 24^{\prime} \mathrm{N}, 12^{\circ} 0^{\prime} \mathrm{E}, 4$ Aug 1865 Ball s. n. (US); Judikarien, Gavardina-Pass, $45^{\circ} 58^{\prime} \mathrm{N}, 10^{\circ} 45^{\prime} \mathrm{E}$, Aug 1925 , Beger s. $n$. (B); Belluno province, Canazei, langs de Avisio, $46^{\circ} 27^{\prime} \mathrm{N}, 12^{\circ} 0^{\prime} \mathrm{E}, 25 \mathrm{Jul}$ 1959, Boom s. n. (L); Verona province, E Monte Baldo, in glareosis Giari di Valbrutta vocatis, $45^{\circ} 43^{\prime} \mathrm{N}, 10^{\circ} 49^{\prime} \mathrm{E}, 29$ Jun 1880 , Goiran s. $n$. (F, L, M, WU)

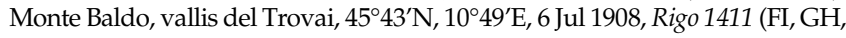
$\mathrm{K}, \mathrm{LE}, \mathrm{W})$; Rocca Pietore, le long du chemin qui va à Sottoguda, $46^{\circ} 25^{\prime} \mathrm{N}$,

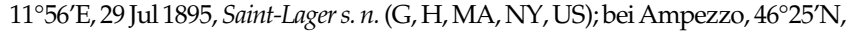
$12^{\circ} 47^{\prime} \mathrm{E}$, Aug 1863, Sonklar s. n. (W); Prov. Verona, Monte Baldo Wetseite, Abstieg, nach Malcesine, $45^{\circ} 43^{\prime} \mathrm{N}, 10^{\circ} 49^{\prime} \mathrm{E}, 17 \mathrm{Jul} 1910$, Frimmel s. n. (W).

SLOVENIA. Gorenjska: Julijske Alpe, Ponca, Na Vrtcu, $46^{\circ} 27^{\prime} \mathrm{N}$, $13^{\circ} 42^{\prime} \mathrm{E}, 14$ Aug 2008, Frajman s. n. \& Schönswetter (WU); Julische Alpen, Wochein [Bohinj], 46 $18^{\prime} \mathrm{N}$, 13 ${ }^{\circ} 56^{\prime} \mathrm{E}$, Freyer s. n. (M); Karawanken, S Loiblpass, Westteil der Kosuta (Koschuta), WNW unter der Kote 1602 bzw. $0,3 \mathrm{~km}$ S Pri Jurji, $46^{\circ} 25^{\prime} \mathrm{N}, 1^{\circ} 25^{\prime} \mathrm{E}, 18 \mathrm{Jul} 1995$, Greimler s. $n$. (WU); Julische Alpen, Massiv des Triglav, am Dom na Komni westlich des Wocheiner Sees, $46^{\circ} 22^{\prime} \mathrm{N}, 13^{\circ} 50^{\prime} \mathrm{E}, 30 \mathrm{Jul} 1972$, Holtz s. n. (GOET). Goriska: Alpi Giulie, Santa Lucia, $46^{\circ} 8^{\prime} \mathrm{N}, 13^{\circ} 44^{\prime} \mathrm{E}$, Aug 1897, Marchesetti s. n. (FI); Selva di

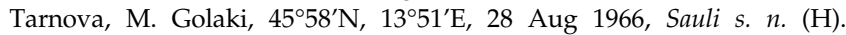
Savinjska: Styriae montibus Sulzbacher Alpen, in sinu Logarthal, $46^{\circ} 23^{\prime} \mathrm{N}$,

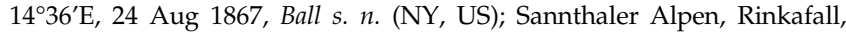
Logarthal, 46 $23^{\prime} \mathrm{N}, 14^{\circ} 37^{\prime} \mathrm{E}, 22$ Aug 1879, Dingler s. n. (GOET).
Notes-Trisetum argenteum is separated from T. distichophyllum because of its more slender habit, narrower and more slender leaves (basal leaf blades (0.9-)1.2-1.5(-1.7) $\mathrm{mm}$ wide), larger panicles, always 1-veined lower glume, and shorter callus hairs ((0.7-)1.4-1.8(-2.6) $\mathrm{mm}$ long). In the studied material, some intermediate specimens were found, mostly in the Central and Eastern Alps. The panicle shape and the number of nerves in the lower glume are highly variable, with $T$. distichophyllum having 1-3 nerves, a character that could be related to the size of the glume. In his work on the cytology and distribution of T. distichophyllum and T. argenteum, Beuret (1974) separated tetraploid (mostly in the Western Alps), octoploid (Eastern Alps), and hexaploid (dispersed in both the Western and Eastern Alps) races of T. distichophyllum. Trisetum argenteum was always tetraploid. Comparing this cytological information with the morphology of the plants, Beuret concluded that these three races are very closely related. According to his hypothesis, the tetraploid populations were separated because of the glaciations, and are real vicariants. However, our morphological data do not support the taxonomic separation of these entities.

3. Trisetum macrotrichum Hack., Magyar Bot. Lapok 2: 110. 1903. Trisetaria macrotricha (Hack.) Banfi \& Soldano, Atti Soc. Ital. Sci. Nat. Mus. Civico Storia Nat. Milano 135: 383. 1996.-TYPE: ROMANIA. Timis river, $45^{\circ} 48^{\prime} \mathrm{N}, 25^{\circ} 39^{\prime} \mathrm{E}$, 17 Aug 1881, J. Barth s. n. (lectotype, here designated, LD-1213569!)

T. tarnowskii Zapal., Rozpr. Wydz.Mat.-Przyr. Akad. Umiejetn., Dzial B, Nauki Biol., III, 4: 167. 1904.—SYNTYPES: ROMANIA. in montanis Bucovinae, monte Dadul, prope Kirlibaba, $47^{\circ} 34^{\prime} \mathrm{N}, 2^{\circ} 7^{\prime} \mathrm{E}$, F. Herbich s. n.; monte Pietrile Domnei (Piatra Domnei) prope Rareu, $45^{\circ} 23^{\prime} \mathrm{N}, 2^{\circ} 46^{\prime} \mathrm{E}$, A. Rehmann s. n. (no original material found).

Herbs (33-)38.5-67(-77) cm tall, not tufted, stoloniferous rhizomes, culms $0.6-1.1 \mathrm{~mm}$ diam, glabrous, sometimes slightly puberulous at the top, with hairs up to $0.1 \mathrm{~mm}$ long; nodes $4-6(-7)$, separated along the culm, enclosed by the sheaths, sometimes the upper ones not enclosed, glabrous or puberulous. Basal leaf sheaths densely pubescent, with hairs 0.9-1.6 $\mathrm{mm}$ long, decaying into fibers, brownish, sometimes yellowish; basal leaf blades (3-)3.8-5.7(-6.5) cm long $\times$ (2.4-)2.6-5.4(-5.6) $\mathrm{mm}$ wide, flat, sometimes inrolled, pubescent to slightly pubescent or with scattered hairs abaxially, densely pubescent adaxially, margins with hairs (0.1-)0.5-1.1(-1.4) $\mathrm{mm}$ long, brownish to greenish; top culm leaf sheaths (9.3-)9.7-17.5(-18) cm long, slightly longer than the internodes, sometimes shorter, glabrous, rarely scabridulous, with cilia along the margins; top culm leaf blades (5.3-)5.4-7.8(-11.3) cm long $\times(4.4-) 5-7.2(-10) \mathrm{mm}$ wide, flat, rarely conduplicate, glabrous to slightly pubescent abaxially and adaxially, with hairs on the margins (0.1-)1-1.6(1.8) mm long, greenish; inner collar region with the same indumentum as the leaf blade or sheath, with hairs 0.05-1.2(-1.7) $\mathrm{mm}$ long; ligules 1-1.3(-1.5) $\mathrm{mm}$ long, slightly laciniate, rarely slightly dentate, glabrous to pubescent, with hairs $0.1-0.3 \mathrm{~mm}$ long. Basal node of the panicle glabrous, rarely scabridulous, with hairs $0.2 \mathrm{~mm}$ long. Panicles (9)9.2-12.2(-15) cm long $\times(2-) 2.5-5.5(-6) \mathrm{cm}$ wide, oblong to elliptic in outline, rarely narrowly oblong to narrowly elliptic; rachis scabridulous to pubescent, with hairs up to $0.15 \mathrm{~mm}$ long; longest basal branches (1.3-)1.6-3(-3.5) cm. Spikelets (5.6-)6-8(-8.2) $\mathrm{mm}$ 


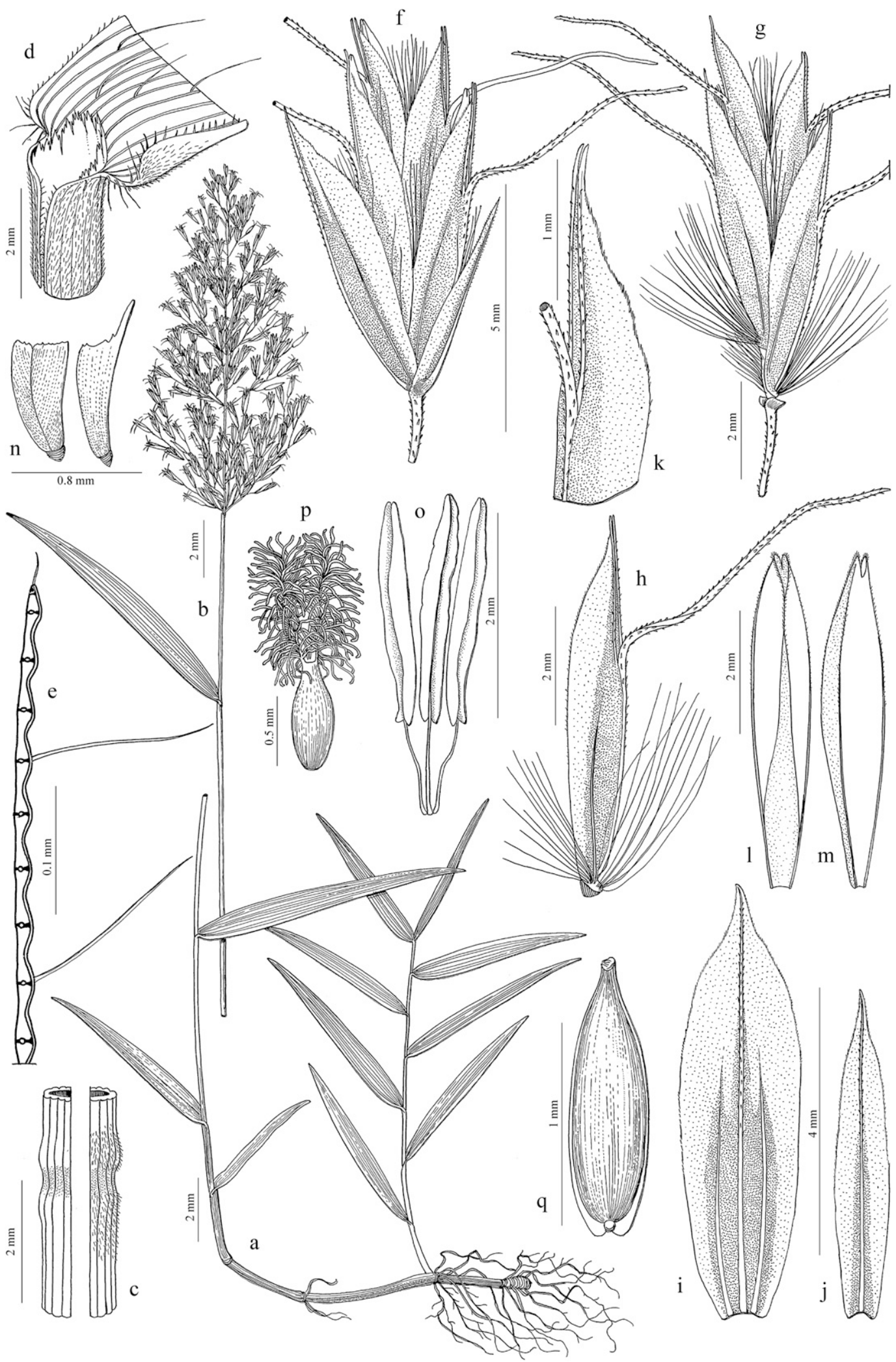

FIG. 4. Trisetum macrotrichum. A. Habit. B. Inflorescence. C. Portions of the culm and node. D. Sheath, ligule, and portion of the blade. E. Transverse section of half of the leaf blade. F. Spikelet. G. Florets. H. Floret. I. First glume, dorsal view. J. Second glume, dorsal view. K. Lemma, upper part, lateral view. L. Palea, ventral view. M. Palea, lateral view. N. Lodicules. O. Stamens. P. Pistil. Q. Caryopsis. (Degen 160, JE, O-V2126613, A-P; Hermann s. n., B-100526317, N, Q).

long $\times(2.5-) 3.7-4.85(-5.2) \mathrm{mm}$ wide, (2-)3-4-flowered; pedicels 2.8-3.5 mm long, pubescent, with hairs $0.1(-0.2) \mathrm{mm}$ long. Glumes unequal (ratio lower glume length/upper glume length $=(0.58-) 0.62-0.72(-0.78))$; lower glume 3.2-4.6(-6) $\mathrm{mm}$ long $\times(0.6-) 0.7-1(-1.2) \mathrm{mm}$ wide, narrowly lanceolate, rarely narrowly elliptic (ratio lower glume width/lower glume length $=$ (0.19-)0.2-0.22(-0.25)), acuminate to long acuminate, 1(-2)nerved, glabrous, with very short hairs on the midrib up to 


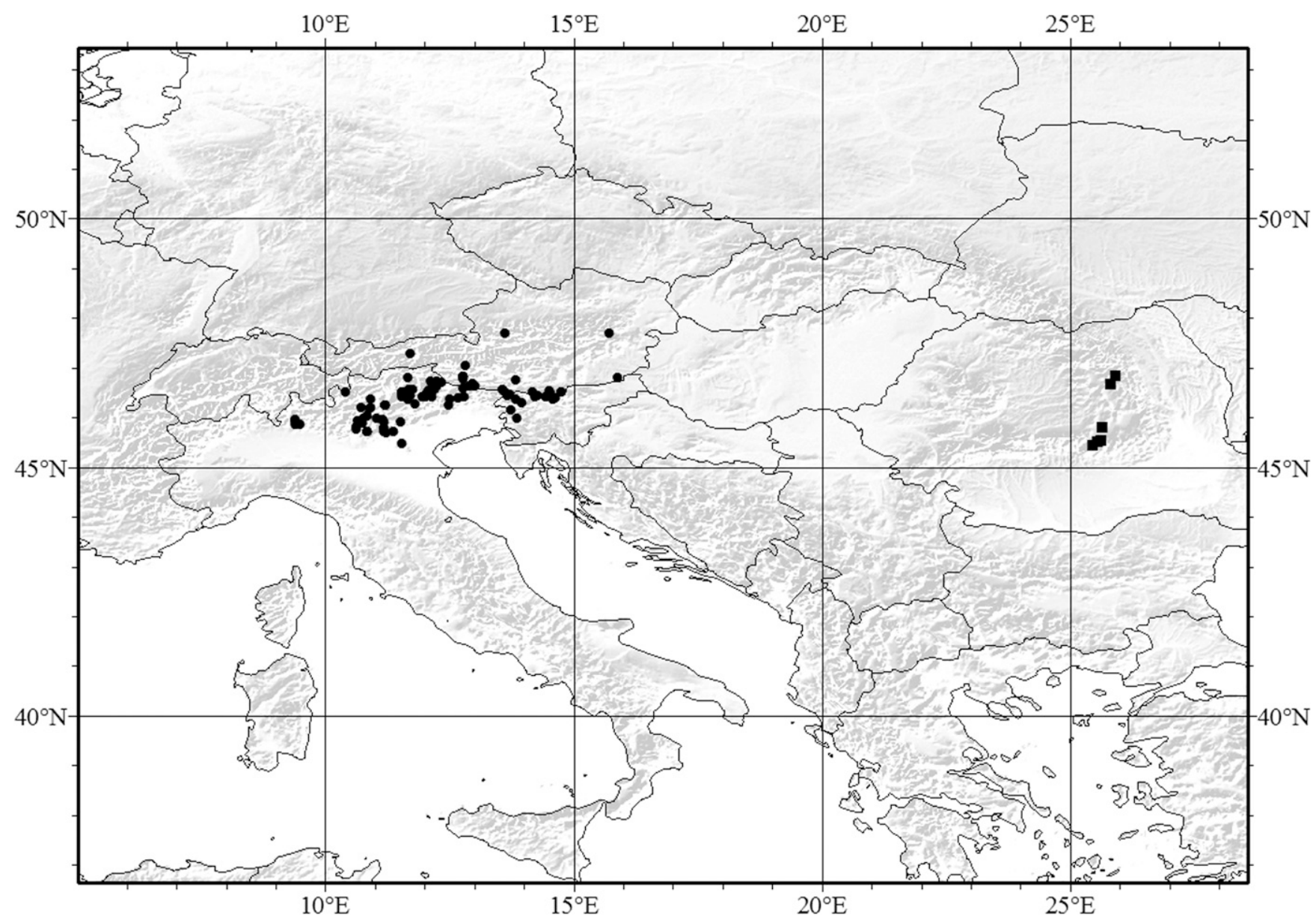

FIG. 5. Distribution of Trisetum argenteum (dots) and T. macrotrichum (squares).

$0.1 \mathrm{~mm}$ long, sometimes on the upper part of the margins, greenish to yellowish on the central part, rest hyaline; upper glume (4.8-)5.2-6.7(-7.7) $\mathrm{mm}$ long $\times 1.9-2.2(-2.4) \mathrm{mm}$ wide, elliptic to oblong, sometimes oblanceolate or narrowly lanceolate (ratio upper glume width/upper glume length $=$ (0.29-)0.31-0.38(-0.42)), acuminate to long acuminate, 3-nerved, glabrous, with very short hairs up to $0.07 \mathrm{~mm}$ long, from the middle to the upper part of the central nerve, sometimes also on the upper part of the margins, greenish to yellowish on the central basal part, rest hyaline; rachilla segment between first and second floret (0.6-)0.7-1.1 mm long, with hairs 3-3.7(-4.6) $\mathrm{mm}$ long; rachilla segments to sterile floret $1-1.5 \mathrm{~mm}$ long, with hairs (1.2-)1.7-2(-2.3) mm long. Lemmas (5-)5.5-6.1(-6.7) mm long $\times(0.6-) 0.7-1(-1.2) \mathrm{mm}$ wide, elliptic to broadly lanceolate (ratio lemma width/lemma length = (0.23-)0.26-0.3(-0.34)), scabridulous, sometimes with hairs up to $0.1 \mathrm{~mm}$ long from the central to the upper part of the midrib, greenish to yellowish; apical teeth (0.2-)0.3-0.4(-0.6) $\mathrm{mm}$ long, with aristules $0.3-0.4 \mathrm{~mm}$ long; awn (4.5-)5.3-6.7 $\mathrm{mm}$ long, inserted (2.2-)2.6-3(-3.7) $\mathrm{mm}$ from the base (ratio awn insertion from the base length/lemma length $=(0.42-) 0.44-0.5(-0.61))$, geniculate and slightly twisted near the base, with very short adpressed hairs $0.05-0.1 \mathrm{~mm}$ long; callus $0.2-0.4 \mathrm{~mm}$ long, oblong to elliptic, rarely rounded, with hairs (2.6-)2.8-3.8 mm long. Paleas 4-5 mm long $\times 1-1.3(-1.5) \mathrm{mm}$ wide (ratio palea length/ lemma length $=(0.71-) 0.73-0.8)$, narrowly elliptic or elliptic to oblong, with short hairs along the outer edges; keels with short hairs on the apex, ending in teeth $0.1-0.2 \mathrm{~mm}$ long. Lodicules
(0.7-)0.8(-1.1) mm long, with apex regularly toothed, sometimes with a lateral lobe. Anthers (1.6-)2-2.2(-2.5) mm long. Ovary (0.7-)0.9-1(-1.2) mm long, glabrous. Caryopsis ca. $1.5 \mathrm{~mm}$ long $\times 0.5 \mathrm{~mm}$ wide. Figure 4 .

Chromosome Number-2n= 56 (Frey 1991).

Phenology-Flowering from June to September.

Distribution and Habitat-This species is endemic to the Romanian Carpathians, in the region of Transylvania; at 800-1,600 m elevation; around limestone rocks and pebbles. Figure 5.

Specimens Examined-ROMANIA. Transylvania: Comit. Csik, in valle

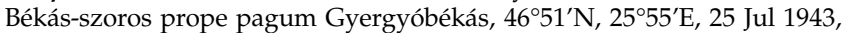
Baschant s. n. (B, GB, HBG, M, S); Comit. Brassó, prope Felsö-Tömös, $45^{\circ} 31^{\prime} \mathrm{N}, 2^{\circ} 34^{\prime} \mathrm{E}, 10$ Jun 1904 , Degen s. n. (C); Tömös, $45^{\circ} 48^{\prime} \mathrm{N}, 25^{\circ} 39^{\prime} \mathrm{E}$, Jun 1904, Wagner s. n. (GB, PR); Comitat Brassó, am Fusse des Berges Piatra, $45^{\circ} 33^{\prime} \mathrm{N}, 2^{\circ} 38^{\prime} \mathrm{E}, 20$ Jul 1904, Degen s. n. (B, C, G, GB, H, IBF, K, L, LE, MA, MO, O, P, PR, S, US, W); Comit. Brassó, prope Felsö-Tömös, 453'ㅅN, 253'ㄹ, 15 Jun 1905, Degen s. n. (B, FI, MA, P); Comit. Brassó, ad pedem

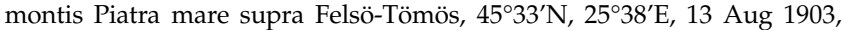
Degen s.n. (B, BM, G, GB, GH, JE, K, LE, O, S, W); Tömös, $45^{\circ} 48^{\prime} \mathrm{N}, 25^{\circ} 39^{\prime} \mathrm{E}$, Mar 1904, Wagner s. n. (PR); Brasov [Kronstadt], Tömös, $45^{\circ} 48^{\prime} \mathrm{N}, 25^{\circ} 39^{\prime} \mathrm{E}$, 25 Jun 1909, Hermann s. n. (B); Carpatti Orientali, Muntii Hasmasul Mare, Umgebung der Schutzhütte Cabana Piatra Singuratica und Bergrücken gegen den Gipfel des Berges Hasmasul Mare (Nagy Hagymás), $46^{\circ} 40^{\prime} \mathrm{N}$,

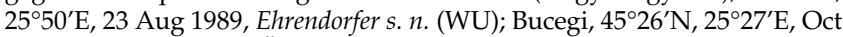

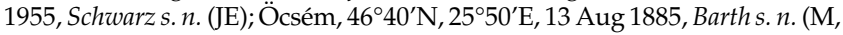
P). [unknown locality], Jul 1909, Hermann s. n. (JE).

Notes-Chrtek (1965) already included Trisetum macrotrichum in sect. Rigida, together with T. rigidum. Apart from the anatomical characters, these species share \pm short ligules, longer rachilla hairs, and young culms with distichous leaves. 
Trisetum macrotrichum is easily differentiated because of the notable width of basal leaf blades $((2.4-) 2.6-5.4(-5.6) \mathrm{mm}$ wide) and flat top culm leaf blades ((4.4-)5.1-7.2(-10) $\mathrm{mm}$ wide) with ciliate margins, with the internodes evenly distributed along the culm, and callus indumentum length ((2.6-)2.8-3.8 mm long).

Trisetum macrotrichum has been misidentified as Avena carpatica auct., non Host (1809) and T. distichophyllum auct., non (Vill.) P. Beauv. (1812). Sãvulescu (1972) noticed that T. distichophyllum has been cited from Romanian Carpathians in floristic literature and stated that both $T$. macrotrichum and $T$. distichophyllum are vicariant taxa and easily confused. Apart from the different size of the plants, both species are easy to differentiate using the aforementioned characters.

4. Trisetum Rigidum (M. Bieb.) Roem. \& Schult., Syst. Veg. 2: 662. 1817. Avena rigida M. Bieb., Fl. Taur.-Caucas. 1: 77. 1808. Avena brevifolia Schrad. ex Spreng., Syst. Veg. (ed. 16) 1: 334. 1824, nom illeg. Trisetaria rigida (M. Bieb.) Banfi \& Soldano, Atti Soc. Ital. Sci. Nat. Mus. Civico Storia Nat. Milano 135: 385. 1996.-TYPE: AZERBAIJAN. Baku: Kuba district, Schirvaniensi altiore ca. Kurt-Bulak [Gora Kara-Bulag] lecta, $41^{\circ} 04^{\prime} \mathrm{N}, 48^{\circ} 20^{\prime} \mathrm{E}$, F.A. Marschall von Bieberstein s. n. (lectotype, designated by Tzvelev (1976: 261), LE-1009635!).

Herbs (18.5-)37-57(-76.3) cm tall, not to loosely tufted, short rhizomatous or with stoloniferous rhizomes, culms 0.4-0.9(-1.2) mm diam, glabrous; nodes (4-)5-7(-12), internodes, sometimes concentrated in lower part, not enclosed by the sheaths, puberulous with hairs $0.3-0.6 \mathrm{~mm}$ long, or sometimes glabrous. Basal leaf sheaths densely pubescent, rarely glabrous, with hairs (0.1-)0.3-0.7(-1.5) mm long, becoming fibrous in age, brownish to yellowish; basal leaf blades $(0.8-) 2.8-5.5(-8.6) \mathrm{cm}$ long $\times(-1.2) 2-3.5(-7.6) \mathrm{mm}$ wide, rigid or not, flat, sometimes slightly inrolled, from glabrous to pubescent abaxially and adaxially, with hairs (0.05-)0.2-0.6(-1) $\mathrm{mm}$ long along the margins, greenish to greyish; top culm leaf sheaths (5.3-)10-13.4(-17) cm long, shorter than the internodes, rarely longer, glabrous, without cilia on the margins; top culm leaf blades (1-)2.5-5(-10.7) cm long $\times(1-) 1.8-3.3(-6.4) \mathrm{mm}$ wide, flat, usually parallel to the culm, glabrous or with scattered hairs abaxially and adaxially, margins without or with hairs (0.05-)0.1-1(-1.5) mm long, greenish to greyish; inner collar region glabrous, sometimes with scattered hairs along the margins (0.1-)0.3-1(-1.3) mm long; ligules (1.3-)2-3.1(-4.2) $\mathrm{mm}$ long, laciniate, glabrous, usually with few hairs (0.05-)0.1-0.4 mm long on the apex. Basal node of the panicle glabrous. Panicle (5.8-)7.5-13(-16) cm long $\times(1.3-) 2.2-3.2(-5.5) \mathrm{cm}$ wide, oblong to elliptic in outline, rarely lanceolate, lax to slightly dense; rachis glabrous, sometimes slightly pubescent, with hairs up to $0.1 \mathrm{~mm}$ long; longest basal branches $(0.4-) 1-2.3(-5) \mathrm{cm}$. Spikelets (5.3-)7.5-9.1(-11.5) mm long $\times(1.6-) 3.2-5(-6.2) \mathrm{mm}$ wide, 2-3(-4)-flowered; pedicels (1.6-)3.3-4.3(-5) $\mathrm{mm}$ long, glabrous to pubescent, with hairs up to $0.1 \mathrm{~mm}$ long. Glumes unequal (ratio lower glume length/upper glume length = (0.45-)0.55-0.66(-0.83)); lower glume (2.4-)3.7-5.2(-6.2) $\mathrm{mm}$ long $\times(0.4-) 0.7-0.9(-1.2) \mathrm{mm}$ wide, lanceolate to narrowly elliptic (ratio lower glume width/lower glume length = (0.11-)0.15-0.22(-0.26)), acuminate, rarely acute, 1-nerved, glabrous, with scattered hairs up to $0.08 \mathrm{~mm}$ on the nerve and upper part, greenish on the central part, surrounded by yellowish or hyaline part; upper glume (5.3-)6.6-8(-10.2) $\mathrm{mm}$ long $\times(1.4-) 1.7-2(-2.4) \mathrm{mm}$ wide, oblong to elliptic, rarely lanceolate or oblanceolate (ratio upper glume width/upper glume length $=(0.19-) 0.23-0.28(-0.32))$, acuminate or long acuminate, rarely acute, 3-nerved, glabrous, with very short hairs up to $0.08 \mathrm{~mm}$ from the middle to the upper part of the central nerve, sometimes also on the upper part of the margins, greenish on the central part, surrounded by yellowish or hyaline part; rachilla segment between first and second floret 1-1.5(-2.2) $\mathrm{mm}$ long, with hairs (3.2-)4-4.7(-5.7) $\mathrm{mm}$ long; rachilla segments to sterile floret (0.8-)1.5-2(-2.5) mm long, with hairs (1.6-)2.5-3.3(-4.2) mm long. Lemmas (5.3-)6.4-8.2(-9.3) mm long $\times(0.4-) 0.7-0.9(-1.1) \mathrm{mm}$ wide, narrowly elliptic to narrowly lanceolate, rarely oblong (ratio lemma width/lemma length $=0.18-0.24(-0.28)$ ), strigulose, sometimes with hairs up to $0.1 \mathrm{~mm}$ long on the aristules and midrib, greenish to yellowish, rarely purple; apical teeth (0.1-)0.2-0.5(-1.2) $\mathrm{mm}$ long, with aristules (0.3-)0.4-1(-1.7) mm long; awn (4.3-)5.7-7.3(-8.6) $\mathrm{mm}$ long, inserted (2.4-)3.2-4.2(-5.1) $\mathrm{mm}$ from the base (ratio awn insertion from the base length/lemma length $=(0.4-) 0.45-$ $0.55(-0.62))$, recurved near base to straight, not twisted to slightly twisted, with very short adpressed hairs $0.05 \mathrm{~mm}$ long; callus $0.2-0.4(-0.5) \mathrm{mm}$ long, elliptic to oblong, with hairs (0.3-)0.6-0.9(-1.4) mm long. Paleas (4-)4.9-5.8(-6.6) $\mathrm{mm}$ long $\times$ 1-1.3(-1.8) $\mathrm{mm}$ wide (ratio palea length/lemma length = (0.54-)0.66-0.81(-0.87)), narrowly elliptic to oblong, rarely slightly oblanceolate, with short hairs along the outer edges; keels with short hairs on the apex, ending in teeth (0.1-)0.2$0.3(-0.5) \mathrm{mm}$ long. Lodicules (0.4-)0.6-0.7(-0.9) $\mathrm{mm}$ long. Anthers (1.8-)2.3-2.8(-3.4) mm long. Ovary (0.4-)0.6-0.9(-2.1) mm long, glabrous, rarely with few hairs $0.1-0.3(-0.6) \mathrm{mm}$ long or slightly puberulous on the apex. Caryopsis $1.4-3 \mathrm{~mm}$ long $\times$ 0.3-0.4 mm wide.

a. subsp. RIGIDUM

Arundo wilhelmsii Ledeb., Mem. Acad. Sci. Petersb. 6: 593, Table 19. 1818. Avena wilhelmsii (Ledeb.) Spreng., Syst. Veg. 1: 333. 1824. Trisetum wilhelmsii Ledeb. ex Schult. in J.J. Roemer \& J.A. Schultes, Mant. 2: 367.1824.-TYPE: GEORGIA. Ex Iberia, C. Wilhelms s. n. (lectotype, designated by Tzvelev (1976: 261), LE!; isolectotypes: P-2255872!).

Avena daenensis Boiss., Diagn. Pl. Orient. 7: 123. 1846. Trisetum daenense (Boiss.) Bal., Bull. Soc. Bot. France 21: 14. 1874.TYPE: IRAN. Kuh-e Dana Mountain, Dscheschme-Pias Bsource, $30^{\circ} 44^{\prime} \mathrm{N}, 51^{\circ} 36^{\prime} \mathrm{E}, 29$ Jul 1842, K.G.T. Kotschy $754 a$ (lectotype, here designated, P-2255864!; isolectotypes, BM-959384! G-176318!, P-2255902!).

T. teberdense var. brevifolium Kharadze, Zametki Sist. Georg. Rast. (Tbilisi), 1: 16. 1938.-TYPE: GEORGIA. SamegreloZemo Svaneti: Fontaine Chalde-čalai, $42^{\circ} 42^{\prime} \mathrm{N}, 42^{\circ} 12^{\prime} \mathrm{E}, 9$ Aug 1931, A.L. Kharadze s. n. (lectotype, here designated, TBI (image!)).

T. rigidum subsp. demavandense Chrtek, Acta Univ. Carol., Biol. 1967: 96. 1968.-TYPE: IRAN. Teheran, in the country near the city, $35^{\circ} 42^{\prime} \mathrm{N}, 51^{\circ} 25^{\prime} \mathrm{E}$, K.G.T. Kotschy 390 (holotype, BM-1134941!; isotypes, LE!).

T. persicum Chrtek, Acta Univ. Carol., Biol. 1967: 98. 1968.-

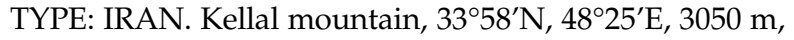
6 Sept 1868, H.C. Haussknecht s. n. (holotype, BM-959383!; isotypes, K-808701!, LE!).

Herbs (18.5-)34.3-56.5(-76.3) cm tall. Basal leaf sheaths densely pubescent, rarely glabrous, with hairs (0.1-)0.3-0.7(-1) mm long; 
basal leaf blades (0.8-)2.8-5.2(-7) $\mathrm{cm}$ long $\times(1.2-) 2-3(-5.8) \mathrm{mm}$ wide, rigid, glabrous abaxially and adaxially, sometimes slightly pubescent, margins with hairs 0.05-0.6(-1) mm long; top culm leaf sheaths (5.3-)9-13(-17) cm long, shorter than the internodes, sometimes slightly longer; top culm leaf blades (1-)2.5-4.6 $(-10.7) \mathrm{cm}$ long $\times(1-) 1.8-3(-5.6) \mathrm{mm}$ wide, divergent, rarely erect, not reaching into the panicle, glabrous abaxially and adaxially, usually with hairs up to $0.4 \mathrm{~mm}$ long on the margins; inner collar region glabrous, rarely with few hairs on the margins 0.1-0.8 mm long; ligules (1.8-)2.3-3.4(-4.2) mm long. Panicles (5.8-)8.1-13.9(-16) cm long $\times(1.3-) 2.3-3.2(-5.5) \mathrm{cm}$ wide, oblong to oblong-elliptic in outline, sometimes lanceolate; longest basal branches $(0.4-) 1-2.3(-5) \mathrm{cm}$. Spikelets (5.3-)7.4-9 $(-11.5) \mathrm{mm}$ long $\times(1.6-) 3.2-5(-6.2) \mathrm{mm}$ wide; pedicels (1.6-) $3.4-4.2(-5) \mathrm{mm}$ long, glabrous to slightly pubescent. Glumes unequal (ratio lower glume length/upper glume length = (0.45-)0.54-0.63(-0.72)); lower glume (2.4-)3.5-5.2(-6.2) $\mathrm{mm}$ long $\times(0.4-) 0.6-1.2 \mathrm{~mm}$ wide (ratio lower glume width/lower glume length $=(0.11-) 0.14-0.22(-0.26))$, greenish on the central part, sometimes purple, laterals and upper part hyaline; upper glume (5.3-)6.4-8(-10.2) mm long $\times 1.6-2.2(-2.4) \mathrm{mm}$ wide (ratio upper glume width/upper glume length $=(0.2-)$ $0.25-0.29(-0.32))$, greenish on the central basal part, usually also the nerves, laterals hyaline or yellowish, sometimes purple; rachilla segment between first and second floret with hairs (3.2-)4.2-4.6(-5) $\mathrm{mm}$ long. Lemmas (5.3-)6.47.7(-9.3) mm long $\times(-0.4) 0.6-1.1 \mathrm{~mm}$ wide, narrowly elliptic to narrowly lanceolate, sometimes elliptic, rarely oblong (ratio lemma width/lemma length $=0.18-0.24(-0.28)$ ); awn (4.3-)5.5-7(-8.6) mm long, inserted (2.4-)3-4.2(-5.1) $\mathrm{mm}$ from the base (ratio awn insertion from the base length/lemma length $=(0.4-) 0.46-0.53(-0.62))$; callus with hairs (0.3-)0.6$0.9(-1.4) \mathrm{mm}$ long. Lodicules with apex slightly lobulate to denticulate, rarely with long lobules or teeths. Anthers (1.8-)2.52.8(-3.4) $\mathrm{mm}$ long. Figure 6.

Chromosome Number-2n= 28 (Tzvelev 1976).

Phenology-Flowering and fruiting from June to August.

Distribution and Habitat-It is distributed from East Anatolia in Turkey to Caucasus and north-northeast Iran; at 600-3,000 m elevation; on rocky or pebbled slopes, from open to shrubby places, often in riversides. Figure 7.

Specimens Examined-ARMENIA. Ararat: Ararat district, montes “Gegamski khrebet", loco Aruni Dzor, 40¹2'N, 4456'E, 11 Jul 1975, Vasak s. n. (B, K, M, MA). Gegharkunik: pr. Pambak, $40^{\circ} 23^{\prime} \mathrm{N}, 45^{\circ} 32^{\prime} \mathrm{E}, 20$ Jun 2005, Aedo 11798 \& al. (MA); Nor-Bajazet district, in monte Inak-dagh, $40^{\circ} 14^{\prime} \mathrm{N}, 4^{\circ} 52^{\prime} \mathrm{E}, 28 \mathrm{Jul} 1928$, Zedelmejer s. $n$. \& Gejdeman (LE). Kotayk Gekhard, $40^{\circ} 9^{\prime} \mathrm{N}, 44^{\circ} 47^{\prime} \mathrm{E}, 5 \mathrm{Jul}$ 1928, Araratian s. n. (LE). Shirak: Akhuryan district, the Shikarskiy Range, northwest of Dzhadzhur Pass, $40^{\circ} 51^{\prime} \mathrm{N}$, 435'르, 23 Jun 1960, Tzvelev 160 \& Cherepanov (LE). Syunik: Megri district, to the valley of a right tributary of the Megriget River near Vardanadzor, $38^{\circ} 58^{\prime} \mathrm{N}, 46^{\circ} 12^{\prime} \mathrm{E}$, 29 Jun 1965, Egorova 1477 \& al. (LE); montes "Karabakhskoie nagorje", ad orientem versus ab oppido Goris, $39^{\circ} 30^{\prime} \mathrm{N}, 46^{\circ} 20^{\prime} \mathrm{E}$, 25 Jul 1975, Vasak s. n. (K). Vayots Dzor: cordillera Vardenis, cerca de 8 km antes del paso de Selim, 39 $56^{\prime} \mathrm{N}, 4^{\circ} 14^{\prime} \mathrm{E}$, 23 Jun 1900, Herrero 2718 (B, O).

AZERBAIJAN. Nachrespublica, in mte Ljakatach, 10 Aug 1933, Gadzhiev s. n. \& al. (LE). Absheron: Kuba district, ad fl. Kussar-czai supra p. Dshagar, $41^{\circ} 34^{\prime} \mathrm{N}, 48^{\circ} 43^{\prime} \mathrm{E}$, 2 Jul 1899, Alexeenko 1101 (LE); Kuba district, prope St. Kussary, $41^{\circ} 25^{\prime} \mathrm{N}, 48^{\circ} 25^{\prime} \mathrm{E}$, 20 Jun 1899 , Alexeenko 392 (LE); Kuba district, in valle fl. Ata-czai, 23 Jul 1900, Alexeenko 5623 (LE); Schemacha district,

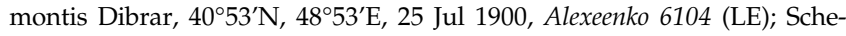
macha district, in decliviis m. Nijal supra p. Zarnova, $40^{\circ} 48^{\prime} \mathrm{N}, 48^{\circ} 20^{\prime} \mathrm{E}, 31$ Jul 1900, Alexeenko 7396 (LE); Baku, Kuba Uyezd, Gilskie polya, 1900, Grigor'ev s. n. (LE); Baku, Bibi-Eibat, $40^{\circ} 18^{\prime} \mathrm{N}, 49^{\circ} 48^{\prime} \mathrm{E}, 24$ May 1912, Holmberg $974(\mathrm{~K}, \mathrm{~S})$; Shemacha district, iter pass pasc. Neregele et Kaladzhich, 30 Jul 1928, Sachokjia s. n. (LE). Aran: Araesch district, Montes Arczan-dagh, Oghrudsha, $41^{\circ} 16^{\prime} \mathrm{N}, 48^{\circ} 0^{\prime} \mathrm{E}$, May 1908 , Schelkownikow s. n. \& Woronow (G, H, LE, MW, W). Daglig-Shirvan: road Agsu-Shamakhi, $40^{\circ} 38^{\prime} \mathrm{N}, 48^{\circ} 28^{\prime} \mathrm{E}, 12$ Jun 1949, Eideman s. n. (LE). Ganja-Qazakh: 20-40 km ENE Tabriz usque $1 \mathrm{~km}$ ultra pontem trans fluvium Talkheh Rud (Atschi Tschai), 38 $4^{\prime} \mathrm{N}, 46^{\circ} 17^{\prime} \mathrm{E}, 31$ May 1971, Rechinger 40731 (G, M, $\mathrm{MO}, \mathrm{S})$. Lankaran: Lerik district, in the vicinity of the urban-type settlement of Lerik, 38 $46^{\circ} \mathrm{N}, 48^{\circ} 24^{\prime} \mathrm{E}, 18$ Aug 1963, Bobrov 574 \& Tzvelev (LE). Nakhchivan: Ordubad Dist., a gorge $1,5 \mathrm{~km}$ east of Ordubad, $38^{\circ} 54^{\prime} \mathrm{N}$, $46^{\circ} 1^{\prime} \mathrm{E}$, 9 Jun 1956, Egorova 433 \& al. (LE); in jugo Zangezur, inter p. Aravsa et monte Dashurry-Dagh, $39^{\circ} 17^{\prime} \mathrm{N}, 45^{\circ} 47^{\prime} \mathrm{E}$, 5 Jul 1928, Gavrilov s. n. (LE); slopes of the Zangezur Range, a slope to the Kyuki-chay River, $39^{\circ} 12^{\prime} \mathrm{N}$, $45^{\circ} 24^{\prime} \mathrm{E}$, 4 Jul 1952, Smol'yaninova s. n. (LE). Shaki-Zaqatala: Zaqatala district, Dzhary, a mount $300 \mathrm{~m}$ west of the mansion of the Zagatala State Reserve, south macroslope, $41^{\circ} 39^{\prime} \mathrm{N}, 46^{\circ} 40^{\prime} \mathrm{E}, 12$ Jun 1946 , Il'inskaya s. $n$. $\mathcal{E}$ Kirpichnikov (LE). Yukhari-Karabakh: Shusha, $39^{\circ} 45^{\prime} \mathrm{N}$, 46 $46^{\circ} \mathrm{E}$, 14 Jun 1893, Lipskiy s. n. (LE).

GEORGIA. Abkhazia: Gudauta district, in vicinitate lacus Rica, $43^{\circ} 28^{\prime} \mathrm{N}$, 40³2'E, 21 Jun 1977, Vasak s. n. (M). Imereti: The Meskhiyskiy Range, [Guminda Ioriy] summit, $42^{\circ} 11^{\prime} \mathrm{N}, 43^{\circ} 36^{\prime} \mathrm{E}, 4$ Aug 1930, Dzens-Litovskaya 99 (LE); Oprtsheti pr. Fl. Rion, $42^{\circ} 21^{\prime} \mathrm{N}, 42^{\circ} 42^{\prime} \mathrm{E}$, Jun 1877 , Brotherus s. $n$. \& Brotherus (H). Kakheti: Greater Caucasus, Tusheti, Pirikiti Khersureti, 42²1'N, 4539'E, 20 Aug 1997, Gagnidze 2524 \& al. (MO); Terek River valley, the Darlyalskoe Gorge, pebbles by "Tamara Castle" (downstream of the mouth of Kistinka river), $42^{\circ} 44^{\prime} \mathrm{N}, 44^{\circ} 37^{\prime} \mathrm{E}, 24$ Aug 1949, Novopokrovskiy 681 (LE); Telav, $41^{\circ} 55^{\prime} \mathrm{N}, 45^{\circ} 28^{\prime} \mathrm{E}, 22$ Jul 1918, Pastuchov s. $n$. (LE, NY). Mtskheta-Mtianeti: Kazbegi region, vill. Targmani area, about $200 \mathrm{~m} \mathrm{~N}$ of vill. Targmani, about $80 \mathrm{~m}$ left of country road, about $50 \mathrm{~m} \mathrm{SW}$ of gas pipeline, $42^{\circ} 39^{\prime} \mathrm{N}, 44^{\circ} 39^{\prime} \mathrm{E}, 7 \mathrm{Jul}$ 2004, Abdaladze 332 \& al. (NY); Kazbegi region, vill. Tsdo area, about $2 \mathrm{~km} \mathrm{SE}$ of vill. Tsdo, $42^{\circ} 41^{\prime} \mathrm{N}, 44^{\circ} 37^{\prime} \mathrm{E}, 7 \mathrm{Aug}$ 2004, Abdaladze 409 \& al. (NY); Georgian Military Road, south slope by the way to the Krestoviy Pass, $42^{\circ} 30^{\prime} \mathrm{N}, 4^{\circ} 27^{\prime} \mathrm{E}, 8$ Aug 1950, Beydeman s. $n$. (LE); inter Tiflis et Wendikaukas, supra stationem Mleti, $42^{\circ} 25^{\prime} \mathrm{N}, 44^{\circ} 29^{\prime} \mathrm{E}$, 10 Aug 1902, Bornmüller 33 (B); upper reaches of the Ksanka River, the left side of a gorge near the selo of Gorga, $42^{\circ} 8^{\prime} \mathrm{N}, 44^{\circ} 24^{\prime} \mathrm{E}, 5$ Aug 1933, Bush s. $n$. \& Bush (LE); between Mlety and Passanauri, $42^{\circ} 21^{\prime} \mathrm{N}, 44^{\circ} 41^{\prime} \mathrm{E}, 28$ Jun 1897 , Fedtchenko s. n. \& Fedtchenko (LE); Greater Caucasus, Khevi, Kazbegi, Karkucha, 42 $34^{\prime} \mathrm{N}, 44^{\circ} 40^{\prime} \mathrm{E}, 25$ Aug 1997, Gagnidze 2941 \& al. (W); prope

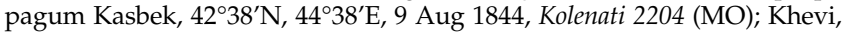
Kazbegi, riv. Tergi Gorge, about $500 \mathrm{~m}$ south of vill. Gergeti, about $200 \mathrm{~m}$ left of riv. Tergi, 42 ${ }^{\circ} 39^{\prime} \mathrm{N}, 4^{\circ} 38^{\prime} \mathrm{E}, 18$ Aug 2001, Nakhutsrishvili 103 \& Abdaladze (NY). Racha-Lechkhumi and Kvemo Svaneti: Kassarskoe Gorge upwards of the village of Tli, left side of the Ardon River, $42^{\circ} 29^{\prime} \mathrm{N}, 43^{\circ} 51^{\prime} \mathrm{E}$, 10 Aug 1939, Shiffers s. n. \& Moreva (LE). Samegrelo-Zemo Svaneti: Gul glacier, $43^{\circ} 4^{\prime} \mathrm{N}, 4^{\circ} 40^{\prime} \mathrm{E}, 21$ Jul 1911, Schelkownikow s. n. (TBI, photo!). Samtskhe-Javakheti: past Abastuman, on the road to the Zekarskiy Pass, $41^{\circ} 45^{\prime} \mathrm{N}$, 42 ${ }^{\circ} 49^{\prime} \mathrm{E}$, 24 Jun 1903, Borodin 197 (LE); Achalzich district, inter Zarzma et Kanly, 41 ${ }^{\circ} 40^{\prime} \mathrm{N}$, 42³9'E, 14 Jul 1914, Litwinow 2809 (C, G, GH, K, M, MW, S, US). South Ossetia: Java district, Ermani area, the south slope of Mt. Fidar-khokh, $42^{\circ} 28^{\prime} \mathrm{N}, 44^{\circ} 14^{\prime} \mathrm{E}, 11$ Sep 1947 , Abramov s. n. (LE); the Chaparukhskoe Gorge, pebbles along the Chaparukh-don River, 23 Jul 1930, Bush s. n. \& Bush (LE); The Sbiyskoe Gorge, the left side of the gorge, slightly downwards of the selo of Nizhnyaya Sba, $42^{\circ} 33^{\prime} \mathrm{N}, 44^{\circ} 10^{\prime} \mathrm{E}$, $30 \mathrm{Jul}$ 1929, Bush s. n. \& Bush (LE); upper course of the Ksanka River, the Sagoy Gorge, near the selo of Bagin, 3 Aug 1930, Bush s. n. \& Bush (LE); Ermani, pebbles on the left side of the Mid. Ermanskaya, $42^{\circ} 31^{\prime} \mathrm{N}, 44^{\circ} 11^{\prime} \mathrm{E}, 20 \mathrm{Aug}$ 1937, Semenova s. n. (LE). Tbilisi: Hänge am Cerepasce-See, 31 May 1968, Fritsch s. n. (JE); Trockene Hügel bei Gladni, $41^{\circ} 49^{\prime} \mathrm{N}, 44^{\circ} 49^{\prime} \mathrm{E}, 14 \mathrm{Jun} 1968$, Fritsch s. n. (JE); Tiflis, $41^{\circ} 41^{\prime} \mathrm{N}, 44^{\circ} 50^{\prime} \mathrm{E}, 10$ Jun 1919, Grossheim 76 (G, LE, MW, PR, S); Tblisi surroundings, environs of Kodjori, Mt. Udzo, ca. 1,5 km $\mathrm{N}$ of Kodjori, ca. $50 \mathrm{~m} \mathrm{~W}$ of the church, $41^{\circ} 40^{\prime} \mathrm{N}, 44^{\circ} 41^{\prime} \mathrm{E}, 18$ Jun 2006 , Lachashvili 189 (NY); Lisi-See, westl. D. Stadt, $41^{\circ} 44^{\prime} \mathrm{N}, 44^{\circ} 44^{\prime} \mathrm{E}$, 30 Jun 1969 , Lepper s. n. \& al. (JE); Akhaltsikhe uyezd, the Zekarskiy Gorge, 20 Jun 1892, Lipskiy s. n. (LE); Tiflis, prope urbem, $41^{\circ} 41^{\prime} \mathrm{N}, 44^{\circ} 50^{\prime} \mathrm{E}, 6 \mathrm{Jul} 1890$, Sommier 1359 \& Levier (FI, LE).

IRAN. Alborz: Mont. Elburs, valle Lur, ad pagum Getschesär, $36^{\circ} 6^{\prime} \mathrm{N}$, 51 $18^{\prime} \mathrm{E}$, 23 Jun 1902, Bornmüller 8383 \& Bornmüller (B). Est Azerbaijan: Isperechan m. Sahand, $37^{\circ} 43^{\prime} \mathrm{N}, 46^{\circ} 29^{\prime} \mathrm{E}, 4$ Aug 1884, Knapp s. $n$. (W). Golestan: M. Shahvar prope Hajjilang, $36^{\circ} 34^{\prime} \mathrm{N}, 54^{\circ} 12^{\prime} \mathrm{E}$, Jul 1948, Rechinger 6091 (B, M). Khorāsān-e Shomāli: Akher-Dagh mountain, 37³4' N, 57 $12^{\prime}$ E, Jul 1907, Haradiian 1619 (S). Mazanderan: Mont. Elburs, Junesar,

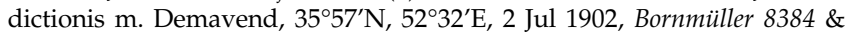
Bornmüller (B); road of Haraz, 18 Jul 1972, Dini-Arazm 12132 (K); Elburs, Pole Zangule, $36^{\circ} 11^{\prime} \mathrm{N}, 5^{\circ} 20^{\prime} \mathrm{E}$, 14 Jun 1937, Gauba 1243 (B); Montes Elburs centr., jugi Kandava [Kandovan], 36 $6^{\circ} 9^{\prime} \mathrm{N}, 51^{\circ} 19^{\prime} \mathrm{E}, 14$ Jun 1937, Gauba s. $n$. (US); ad radices m. Demawend, 35 $57^{\prime} \mathrm{N}, 52^{\circ} 6^{\prime} \mathrm{E}, 24$ Jun 1843, Kotschy 391 (G, LE, P); Kotschy 393 (L, LE, P); in faucibus vallis Chalus supra Valiabad, $36^{\circ} 15^{\prime} \mathrm{N}, 51^{\circ} 18^{\prime} \mathrm{E}$, 21 Jun 1974, Rechinger 48369 (G, M); Nur district, inter Kamarband et jugum Naftab, $36^{\circ} 13^{\prime} \mathrm{N}, 5^{\circ} 17^{\prime} \mathrm{E}, 8$ Aug 1948, Rechinger 


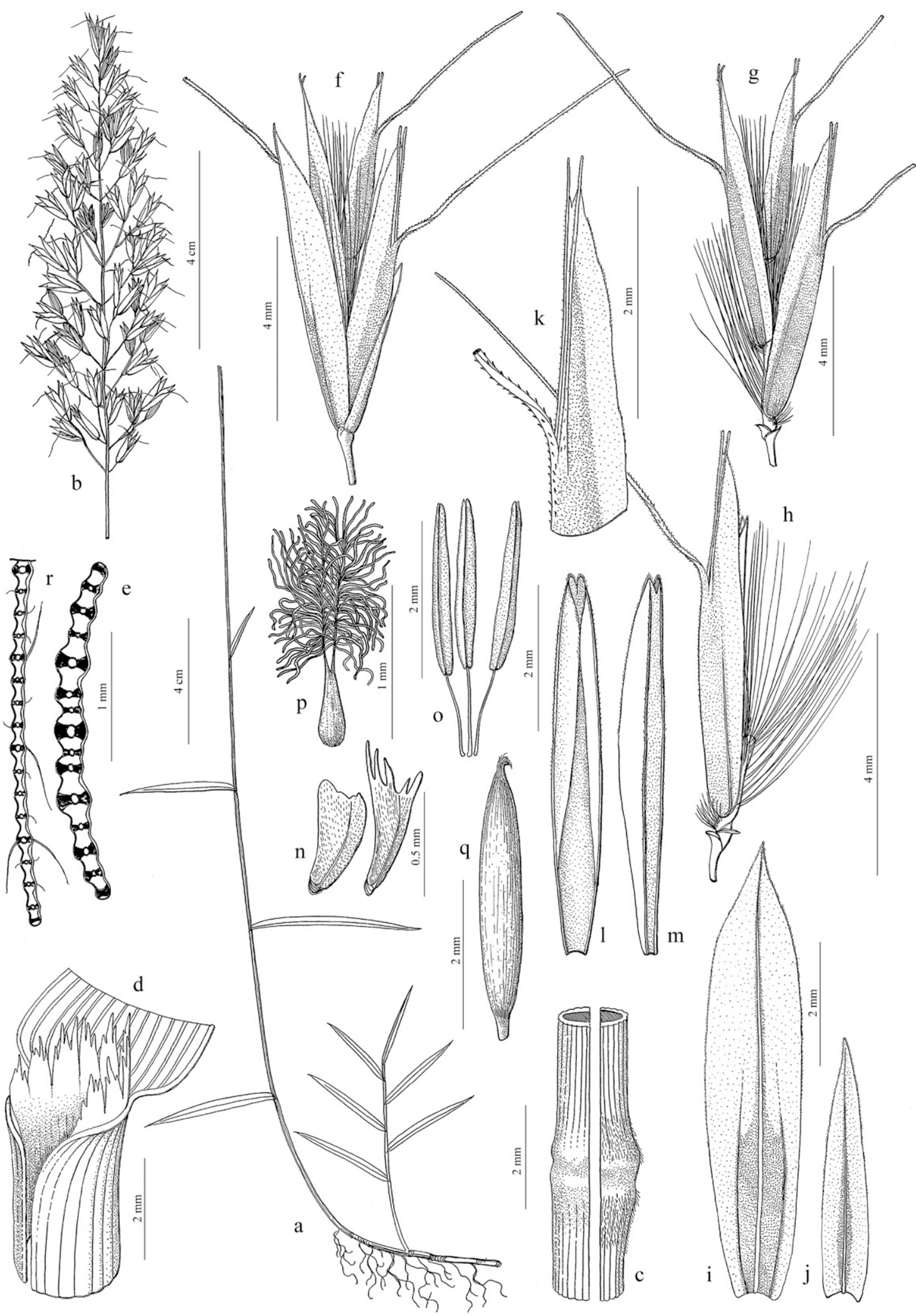

FIG. 6. A-Q. Trisetum rigidum subsp. rigidum. A. Habit. B. Inflorescence. C. Portions of the culm and node. D. Sheath, ligule, and portion of the blade. E. Transverse section of leaf blade. F. Spikelet. G. Florets. H. Floret. I. First glume, dorsal view. J. Second glume, dorsal view. K. Lemma, upper part, lateral view. L. Palea, ventral view. M. Palea, dorsal view. N. Lodicules. O. Stamens. P. Pistil. Q. Caryopsis. R. T. rigidum subsp. teberdense. Transverse section of half of the leaf blade. (Amirhanov 34, MW, A-B; Gauba 1243, B-100526552, C-J, L-P; Alexeenko 9071, LE, N; Vasak s. n., K, Q; Soreng 8009, US-3600681, R).

6447 \& Rechinger (US); Kudjur district, in monte Ulodj, 9 Aug 1948, Rechinger 6509 \& Rechinger (M, US). Qazvin: Montes Elburs centr., Hesarband mountain, $35^{\circ} 50^{\prime} \mathrm{N}, 51^{\circ} 15^{\prime} \mathrm{E}, 24 \mathrm{Jul} 1935$, Gauba s. n. (M, US). Semnan: Shahrud-Bustam district, montium Shahvar supra Nekarman

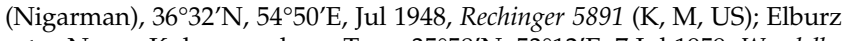
mts., Nezva Kuh area, above Taru, $35^{\circ} 58^{\prime} \mathrm{N}, 53^{\circ} 12^{\prime} \mathrm{E}, 7 \mathrm{Jul} 1959$, Wendelbo 1218 (LE). Tehran: Zentral-Elburs, am Südabhang des Totschal im Tal von

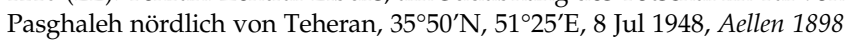




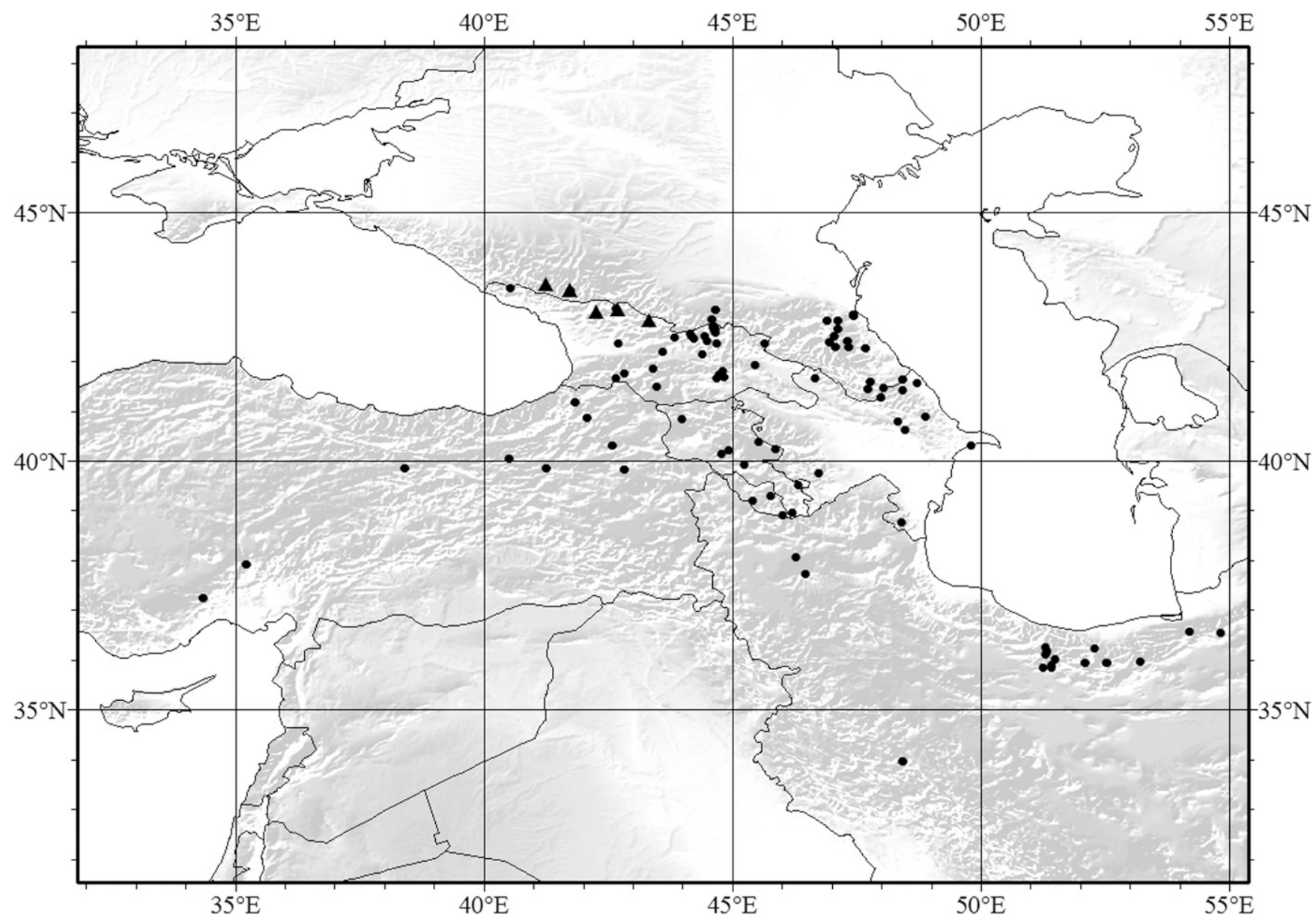

FIG. 7. Distribution of Trisetum rigidum subsp. rigidum (dots) and T. rigidum subsp. teberdense (triangles).

(F, MO, S); Zentral-Elburs, am Südabhang des Totschal im Tal Häfthous

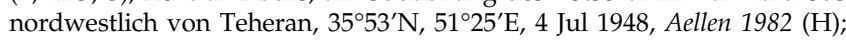
Mont. Elburs, ad basin septentr. Alpium Totschal, prope Scheheristanek, $35^{\circ} 57^{\prime} \mathrm{N}$, 52 $32^{\prime} \mathrm{E}, 15$ Jun 1902, Bornmüller 8381 \& Bornmüller (B, PR); Bornmüller 8382 \& Bornmüller (BM, JE, MPU, W); Mont. Elburs, alpe Totschal, prope Scheheristanek, $35^{\circ} 57^{\prime} \mathrm{N}, 5^{\circ} 32^{\prime} \mathrm{E}, 8$ Jul 1902, Bornmüller 8385 \& Bornmüller (B, HBG, JE, LE, PR, W); 10 Jun 1902, Bornmüller 8385b \& Bornmiüller (B, G); Elburz $24^{\prime} \mathrm{NNE}$ of Teheran, $35^{\circ} 57^{\prime} \mathrm{N}, 52^{\circ} 32^{\prime} \mathrm{E}, 11 \mathrm{Jul} 1962$, Furse 3141 (LE); Montes Elburz, Shemshak, 36 $0^{\circ}$ N , 51 ${ }^{\circ} 29^{\prime} \mathrm{E}, 25$ Apr 1977, Rechinger $57178(\mathrm{M})$.

RUSSIA. Dagestan: Samur district, prope pagum Gedym, $41^{\circ} 38^{\prime} \mathrm{N}$, $48^{\circ} 25^{\prime} \mathrm{E}$, 17 Aug 1900, Alexeenko 9071 (LE); Kürinskii district, prope pagum Kgirchan (Gelchan), 23 Jul 1898, Alexeenko s. n. (LE); Dargi district, prope Murguk, $42^{\circ} 16^{\prime} \mathrm{N}, 47^{\circ} 41^{\prime} \mathrm{E}, 7$ Jul 1898, Alexeenko s. n. (LE); Dargi district, prope silvam Gumra-duz inter Akuscha et Muhi (Meha), $42^{\circ} 17^{\prime} \mathrm{N}$, $47^{\circ} 21^{\prime} \mathrm{W}, 14 \mathrm{Jul} 1898$, Alexeenko s. n. (LE); prope Lewaschi, $42^{\circ} 25^{\prime} \mathrm{N}, 47^{\circ} 19^{\prime} \mathrm{E}$ 2 Jul 1898, Alexeenko s. n. (LE); Dargi district, in declivitate meridionali jugi Shamchal-dag, 5 Jul 1898, Alexeenko s. $n$. (LE); Dargi district, prope pagum Chodshal-makhi, 12 Aug 1898, Alexeenko s. n. (LE); Tenir-Chan-Schura district, inter pag. Ischkarty et Temir-Chan-Schura, $42^{\circ} 49^{\prime} \mathrm{N}, 47^{\circ} 7^{\prime} \mathrm{E}, 30$ Jun 1897, Alexeenko s. n. (LE); Achty, $41^{\circ} 27^{\prime} \mathrm{N}, 4^{\circ} 43^{\prime} \mathrm{E}, 17 \mathrm{Jun} 1880$, Becker s. $n$. (JE, LE); Makhach-kala district, SE slope of Mt. Tarku-Tau, $42^{\circ} 56^{\prime} \mathrm{N}$, $47^{\circ} 27^{\prime} \mathrm{E}$, 8 Jun 1925, Bogdanovskaya-Gienef s. n. (LE); Gunib district, the selo of Chokh, Mt. Turchidag, $42^{\circ} 18^{\prime} \mathrm{N}, 47^{\circ} 4^{\prime} \mathrm{E}, 10$ Aug 1940 , Elenevskiy s. n. (LE); Magaramkentskiy district, the selo of Garakh, slopes of a mount by the Samur River, $41^{\circ} 28^{\prime} \mathrm{N}$, $48^{\circ} 2^{\prime} \mathrm{E}$, 15 Aug 2006, Kotseruba 30 (LE); valley of the Kurakh River, $12 \mathrm{~km}$ downwards of the selo of Kurakh, $41^{\circ} 35^{\prime} \mathrm{N}, 47^{\circ} 46^{\prime} \mathrm{E}$, 13 Jul 1956, Medvedeva s. n. \& al. (LE); Lakskiy district, along the Kazikukhumskoe Koysu River, Kazi-Kukhum, 42 ${ }^{\circ} 30^{\prime} \mathrm{N}, 47^{\circ} 3^{\prime} \mathrm{E}$, 2 Sep 1927, Poretskiy 543 \& Shults (LE); Makhachkala district, the Narat-tyube Range, west of Agach-aul, 42 ${ }^{\circ} 55^{\prime} \mathrm{N}, 4^{\circ} 27^{\prime} \mathrm{E}, 1$ Jul 1955, Prokhanov 339 (LE); Buynaksk district, the Gimrinskiy Range, ca. $1 \mathrm{~km}$ away from a pass (near the aul of Verkhniy Karanay), 42 ${ }^{\circ} 49^{\prime} \mathrm{N}, 4^{\circ} 54^{\prime} \mathrm{E}, 23$ Aug 1953, Prokhanov 163 \& Cheldyshev (LE). Kuban: Teberda, $43^{\circ} 26^{\prime} \mathrm{N}, 41^{\circ} 44^{\prime} \mathrm{E}, 5$ Jul 1905, Litwinow
257 (LE). North Ossetia-Alania: SE slopes of Mt. Kandyl above Dzhekharovskoe (Georgian Military Road), 42 $50^{\prime} \mathrm{N}, 44^{\circ} 36^{\prime} \mathrm{E}, 20$ Jul 1940, Schiffers 5 \& Moreva (LE); valley of the Terek River, $37 \mathrm{~km}$ south of

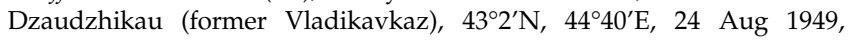
Vasilchenko 668 \& al. (LE).

TURKEY: partie supérieure du Bousdouan-dagh [Bozdoğan], au dessus de Khabakhor (Lazistán), 40 $52^{\circ} \mathrm{N}, 42^{\circ} 5^{\prime} \mathrm{E}, 30$ Aug 1866, Balansa s. n. (L); région alpine du Taurus, au-dessus de Boulgarmaden, $37^{\circ} 14^{\prime} \mathrm{N}$, $21^{\circ} 51^{\prime}$ E, IX, Balansa s. n. (L); Erzurum, Palandöken dagi unmittelbar S der Stadt Erzurum, $0.2 \mathrm{~km}$ oberhalb der Schilift-Talstation, $39^{\circ} 51^{\prime} \mathrm{N}$, 41 ${ }^{\circ} 16^{\prime} \mathrm{E}, 26$ Jul 1978, Ehrendorfer s. n. \& al. (WU); Erzincan/Sivas, Kızıldağ Geçidi, 42 Str.-km W Refahiye, 30 Str. Km E Imranli, 39 $5{ }^{\circ} 51^{\prime} \mathrm{N}, 38^{\circ} 24^{\prime} \mathrm{E}$, 3 Aug 1978, Ehrendorfer s. n. \& al. (WU); Erzurum, zwischen Askale und Bayburt auf dem Kopdagi geçidi steiniger, $40^{\circ} 3^{\prime} \mathrm{N}, 40^{\circ} 30^{\prime} \mathrm{E}, 2$ Aug 1973, Holtz 978 \& al. (GOET); Aladağlar, Emli Bogazi, ca. 1-1.5 km SE des Karasay Tepe, $37^{\circ} 55^{\prime} \mathrm{N}, 35^{\circ} 13^{\prime} \mathrm{E}, 12$ Aug 1992, Parolly s. n. (B); Kars, Sarikamis, am Pass $10 \mathrm{~km}$ westlich der Stadt and der Strasse nach Horasan, 40¹9'N , 42 $35^{\prime}$ E, 23 Jul 1981, Raus 4431 (B); between Erzurum and Agri, $25 \mathrm{~km}$ west of Toprakkale village, Keeitepe mountain, $39^{\circ} 50^{\prime} \mathrm{N}$, $42^{\circ} 49^{\prime} \mathrm{E}, 18 \mathrm{Jul}$ 1976, Tatli 4865 (GOET); Batumi, Artvin district, between the villages of Dabatsvril and Skhloban, $41^{\circ} 10^{\prime} \mathrm{N}, 41^{\circ} 50^{\prime} \mathrm{E}, 16 \mathrm{Jul} 1911$, Woronow 6256 (LE).

b. subsp. TEBERDENSE (Litv.) Tzvelev, Novosti Sist. Vyssh. Rast. 7: 62. 1971.

Trisetum rigidum var. teberdense Litv., Spisok Rast. Gerb. Fl. S.S.S.R. Bot. Inst. Vsesojuzn. Akad. Nauk 9: 11. 1932. Trisetum teberdense (Litv.) Kharadze, Zam. Sist. Georg. Rast. (Tbilisi) 1: 14. 1938.-TYPE: RUSSIA. Kuban Region, Teberda river, $43^{\circ} 26^{\prime} \mathrm{N}, 41^{\circ} 44^{\prime} \mathrm{E}, 10 \mathrm{Jul} 1905$, D.I. Litwinow 2810 (lectotype, designated by Tzvelev (1976: 260), LE!; 
isolectotypes, A!, C!, G-305395!, M-210841!, MW!, S13-32730!, US-2119656!, WISv-262239).

T. rigidum var. ciliatifolium Roshev. in Kom. (ed.), Fl. URSS 2: 256. 1934.-TYPE: [unknown locality and collector] (no original material found).

Herbs (47-)50-67(-72) cm tall. Basal leaf sheaths densely pubescent, with hairs up to $1.5 \mathrm{~mm}$ long; basal leaf blades (5-)7.5-8 $(-8.6) \mathrm{cm}$ long $\times(3.2-) 3.7-5(-7.6) \mathrm{mm}$ wide, not rigid, from glabrous to pubescent abaxially and adaxially, margins with hairs 0.4-1 mm long; top culm leaf sheaths $11-15 \mathrm{~cm}$ long, shorter than the internodes; top culm leaf blades (3.7-)6.3-6.7 cm long $\times(2.6-)$ 3.3-5.7(-6.4) $\mathrm{mm}$ wide, usually erect, sometimes reaching into the panicle, with scattered hairs abaxially and adaxially, margins with hairs 0.05-0.4(-1.5) mm long; inner collar region glabrous, with scattered hairs on the margins (0.4-)0.6-1.3 mm long; ligules $1.3-2.4 \mathrm{~mm}$ long. Panicles $11-15.5 \mathrm{~cm}$ long $\times(2-) 2.8-4 \mathrm{~cm}$ wide, narrowly elliptic to oblong; longest basal branches $2-4.7 \mathrm{~cm}$ long. Spikelets (7.5-)8-9 mm long $\times(2.8-) 3.4-4.6 \mathrm{~mm}$ wide; pedicels $3.5-5 \mathrm{~mm}$ long, pubescent to slightly pubescent. Glumes unequal (ratio lower glume length/upper glume length $=0.57-0.64$ $(-0.83))$; lower glume $4-5.5 \mathrm{~mm}$ long $\times 0.5-0.8(-1) \mathrm{mm}$ wide (ratio lower glume width/lower glume length $=0.13-0.18$ ), greenish on the central part surrounded by yellowish; upper glume $6.6-8.4 \times 1.4-1.8 \mathrm{~mm}$ long (ratio upper glume width/ upper glume length $=0.19-0.26$ ), greenish or sometimes purplish on the central part, surrounded by part hyaline or yellowish; rachilla segment between first and second floret with hairs 4.5-5.7 mm long. Lemmas (6.7-)7-8.5 mm long $\times 0.5-0.8(-1) \mathrm{mm}$ wide, narrowly elliptic to narrowly lanceolate (ratio lemma width/lemma length $=0.14-0.24$ ); awn 7-8.6 mm long, inserted $3.6-4.8 \mathrm{~mm}$ from the base (ratio awn insertion from the base length/lemma length $=0.5-0.6$ ); callus with hairs $0.7-0.8(-1) \mathrm{mm}$ long. Lodicules regularly toothed or with two straight acute lobules. Anthers 1.9-2.3 mm long. Figure 6.

Chromosome Number-Unknown.

Phenology-Flowering and fruiting from June to August.

Distribution and Habitat-Endemic to the Western Caucasus, from Eastern Turkey to Northern-Eastern Iran; at 1,280-2,000 m elevation; in acidic rocks, under middle mountain forest canopy. Figure 7.

Specimens Examined-GEORGIA. Samegrelo-Zemo Svaneti: Caucasus centralis, in vicinitate oppidi Mestia, ad septentrionem versus, $43^{\circ} 2^{\prime} \mathrm{N}$, $42^{\circ} 43^{\prime} \mathrm{E}$, 28 Jul 1979, Vasak s. n. (M); Kutaisi Governorate, the gorge of the Ingur, passage from the commune of Ipari to the commune of Kal, $43^{\circ} 0^{\prime} \mathrm{N}$, $42^{\circ} 16^{\prime} \mathrm{E}, 28 \mathrm{Jul}$ 1911, Shelkovnikov s. $n$. (TBI); the upper reach of the Vanistskali river, Khorogonskiy mountain mass, 25 Aug 1980, Gagnidze s. n. \& Mosulishvili (TBI). Racha-Lechkhumi and Kvemo Svaneti: the upper reach of the Lukhuni river, $42^{\circ} 49^{\prime} \mathrm{N}, 43^{\circ} 20^{\prime} \mathrm{E}, 12 \mathrm{Jul} 1981$, Chelidze s. n. E Shetekouri (TBI).

RUSSIA. Karachay-Cherkessia: Teberda district, Teberda, $43^{\circ} 26^{\prime} \mathrm{N}$ $41^{\circ} 44^{\prime} \mathrm{E}, 15 \mathrm{Jul}$ 1981, Mosulishoili s. n. (MO); Teberda village, Karakul Lake, $43^{\circ} 26^{\prime} \mathrm{N}, 41^{\circ} 45^{\prime} \mathrm{E}$, $30 \mathrm{Jul} 2010$, Soreng 8009 \& al. (US); Teberda reserve, Teberda valley, $43^{\circ} 26^{\prime} \mathrm{N}, 41^{\circ} 44^{\prime} \mathrm{E}$, 30 Jun 1993, Onipchenko s. n. (MW); Zelenchuk district, Arkhyz, Abishira-Akhuba Ridge, $43^{\circ} 33^{\prime} \mathrm{N}, 41^{\circ} 16^{\prime} \mathrm{E}, 15$

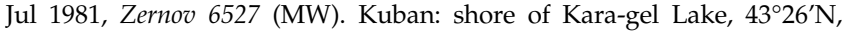
$41^{\circ} 44^{\prime} \mathrm{E}, 1 \mathrm{Jul}$ 1907, Endaurowa s. n. (LE).

Notes-Trisetum rigidum is the most polymorphic taxon of the section and displays great morphological variation throughout its distribution area. The size, as well as the degree of indumentum of the leaf blades and leaf sheaths of this species, is a quite variable character.

Boissier (1846) named some small specimens from southwest Iran as Avena daenesis. Chrtek (1968) described an infraspecific taxon from the Demavand Mountains from northern Iran and named it $T$. rigidum subsp. demavandense
Chrtek; it was separated from T. rigidum s. s. for its smaller size ((8)10-20(-38) cm tall), congested panicles, and larger paleas ((5-)6-7.5 mm long). However, the studied specimens of T. rigidum are extremely variable in size $(15.5-60 \mathrm{~cm}$ tall), as previously noted, and the paleas ((4-)4.5-6(-6.6) $\mathrm{mm}$ long) do not differ significantly from those of $T$. rigidum subsp. demavandense. In specimens from the Elburz area and Demavand Mountains, size variability also occurs, including the type material of $T$. rigidum subsp. demavandense ( $T$. rigidum $\mathrm{f}$. minor in sched.). The easternmost specimens of T. rigidum studied are those collected by Haradjian from the Akher-Dagh Mountains (Haradjian s. n. (B-100526547) and Haradjian 1619 (S)), which are very similar to those from the Demavand Mountains. As previously noted, the size has proved to be a variable character throughout the whole range of the species and has no taxonomic value. For this reason, the smaller specimens have been considered as synonyms of $T$. rigidum.

Chrtek (1968) described Trisetum persicum Chrtek from Luristan, in Southwestern Iran. The main characters used to separate this species are stronger panicles and short rachilla indumentum relative to lemma length. Trisetum persicum has lemmas that are $8.7 \mathrm{~mm}$ long and rachilla hairs that are 3.4-3.7 $\mathrm{mm}$ long, while T. rigidum has lemmas that are (5.3-) 6.4-8.2(-9.3) $\mathrm{mm}$ long and rachilla hairs that are (3.2-)4-4.7 $(-5.7) \mathrm{mm}$ long. It is important to note the morphological distinctiveness of the specimen collected by Archibald 2984 (K) in Eastern Iran (the Bakhtiari region) with regard to the type of T. persicum, which has straight and pubescent leaf blades with hairy margins and spikelets that are $8 \mathrm{~mm}$ long. Although these characters are distinctive, they fall well within their variation range in the species, and therefore we consider $T$. persicum to be a synonym of $T$. rigidum.

Trisetum rigidum var. teberdense Litv. has taller culms, wider leaves with hairs along the margins, and larger spikelets than T. rigidum subsp. rigidum. Kharadze (1938) combined it at the specific level, and after Tzvelev (1971), it has been considered a subspecies. Trisetum rigidum subsp. teberdense specimens have top leaf blades that are (2.6-) 3.3-5.7(-6.4) $\mathrm{mm}$ wide and pilose along the margins, whereas the studied specimens of T. rigidum subsp. rigidum have top leaf blades that are (1-)1.8-3(-5.6) mm wide, abaxially and adaxially glabrous, and sometimes slightly pubescent, with short hairs along the margins. Because of that, we also consider it a subspecies of $T$. rigidum. Moreover, those specimens considered $T$. teberdense present similarities in the disposition of the leaves (parallel to the culm) with $T$. buschianum subsp. buschianum, which are more or less pubescent and with hairy margins; the rest of the characters of the panicle and, more precisely, those of the rest of the spikelets (ratio lower glume/upper glume, callus indumentum, etc.) are the same as in T. rigidum.

Owing to the polymorphism of this species, Kharadze (1938) described a new variety, $T$. teberdense var. brevifolium, here included as synonym of Trisetum rigidum subsp. rigidum. We have only been able to check photos of two of the syntypes. Kharadze's illustration (1938) shows similar traits in the glumes and callus hairs for both taxa. The habit does not differ significantly from specimens of T. buschianum subsp. transcaucasicum, with short top leaf blades, and ovate to ellipticoblong and purplish panicles, but with almost glabrous leaves, and hairs only along the margin as in T. rigidum subsp. teberdense. Further, more detailed studies are needed to verify the taxonomic value of this variety. 


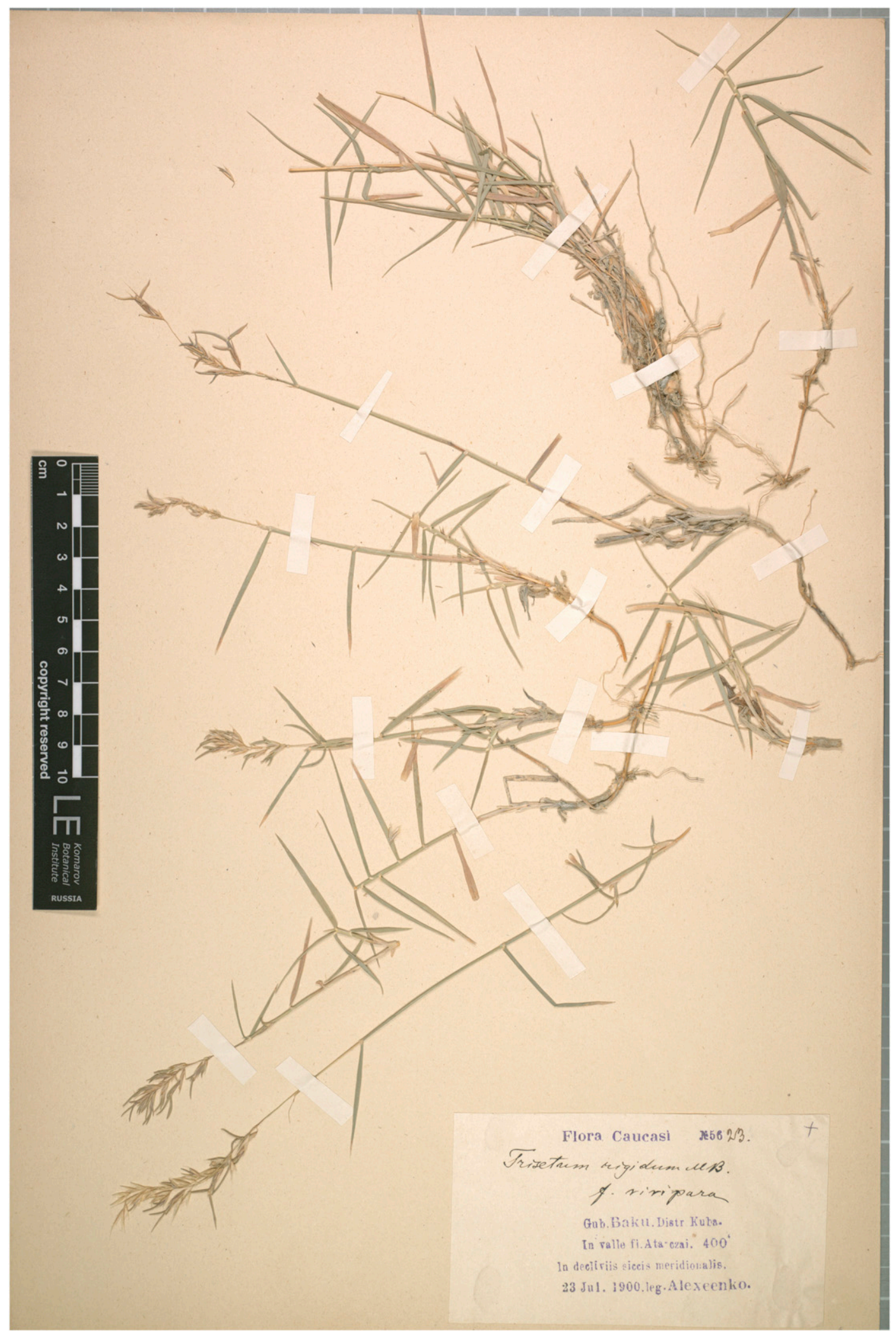

Fig. 8. Trisetum rigidum subsp. rigidum. Specimen with proliferating spikelets, as a possible pseudoviviparous mechanism (Alexeenko 5623, LE).

The tendency of this species to vegetative propagation (f. vivipara in sched.), with the transformation of floral bracts into true leaves, is noticeable in the specimens collected by Alexeenko 5623 (LE) (Fig. 8).
5. TRISETUM Buschianum Seredin, Bot. Mat. (Leningrad) 21: 51. 1961.-TYPE: RUSSIA. Kabardino-Balkar Republic, Besengijskij Czerek, pr. fluminis Besenga, $43^{\circ} 07^{\prime} \mathrm{N}, 43^{\circ} 08^{\prime} \mathrm{E}, 10 \mathrm{Jul}$ 1958, R.M. Seredin s. n. (holotype, LE!; isotype, PGFA). 
Herbs (10.6-)17.2-28.7(-35) cm tall, not or loosely tufted, short rhizomatous or with stoloniferous rhizomes, culms (0.4-) 0.6-0.8(-1.2) mm diam, glabrous; nodes (3-)4-6(-8), separated along the culm or concentrated in lower part, enclosed or not by the sheaths, glabrous. Basal leaf sheaths pubescent to densely pubescent, rarely glabrous, with hairs $0.3-0.7(-1) \mathrm{mm}$ long, yellowish to brownish, sometimes greenish; basal leaf blades (1-)2.5-3.5(-4.7) cm long $\times(1.9-) 2.3-3.2(-5.2) \mathrm{mm}$ wide, flat, sometimes convolute or inrolled, pubescent to densely pubescent abaxially and adaxially, margins with hairs 0.1-0.8(-1.5) $\mathrm{mm}$ long, greenish to greyish; top culm leaf sheaths (3-)4.6-7.6(-10) cm long, shorter or longer than the internodes, glabrous, very rarely with hairs on the upper part, without cilia; top culm leaf blades (1.3-)1.8-5.7(-8) $\mathrm{cm}$ long $\times$ (1.8-)2.5-3.4(-5.2) $\mathrm{mm}$ wide, flat, sometimes conduplicate, parallel or perpendicular to the culm, glabrous to slightly pubescent adaxially and abaxially, margins with hairs (0.05-)0.1-1(-1.3) $\mathrm{mm}$ long, greenish to greyish; inner collar region glabrous, margins with hairs (0.6-)0.9-1.3(-1.8) mm long; ligules (1.2-)1.5-2.1(-3) mm long, laciniate, rarely denticulate, glabrous, sometimes puberulous on the apex, rarely on the surface, with hairs 0.2-0.4(-1.2) $\mathrm{mm}$ long. Basal node of the panicle glabrous. Panicles (3.4-)4-7(-8.6) $\mathrm{cm}$ long $\times$ (1.3-)1.7-2.5(-3.4) cm wide, elliptic to oblong or ovate in outline, rarely lanceolate, lax to slightly dense; rachis glabrous to slightly pubescent, with hairs up to $0.1 \mathrm{~mm}$ long; longest basal branches (0.5-)0.8-1.3(-2.6) cm. Spikelets (5.5-)6.8-7.5(-8.5) mm long $\times(1.7-) 2.7-4.3(-5) \mathrm{mm}$ wide, 2-3flowered; pedicels (1.5-)2-2.8(-4.7) $\mathrm{mm}$ long, glabrous to slightly pubescent, with hairs up to $0.1 \mathrm{~mm}$ long. Glumes unequal to subequal (ratio lower glume length/upper glume length $=(0.69-) 0.72-0.86(-0.94))$; lower glume (4-)4.7-5.5(-6.2) $\mathrm{mm}$ long $\times(0.8-) 1-1.4(-1.8) \mathrm{mm}$ wide, narrowly elliptic to narrowly lanceolate (ratio lower glume width/lower glume length $=(0.15-) 0.21-0.29(-0.31))$, acuminate, sometimes acute or long acuminate, 1-2(-3)-nerved, glabrous, usually with hairs $0.05(-0.2) \mathrm{mm}$ long on the upper part and margins, greenish to purplish; upper glume (5.2-)5.8-6.7(-7.1) mm long $\times(1.4-) 1.8-2(-2.2) \mathrm{mm}$ wide, narrowly elliptic to oblong, rarely lanceolate (ratio upper glume width/upper glume length $=(0.21-) 0.26-0.32(-0.38))$, acuminate, sometimes long acuminate or acute, 3-nerved, glabrous, with very short hairs up to $0.05(-0.2) \mathrm{mm}$ long from the middle to the upper part of the central nerve and upper part of the margins, greenish to purplish; rachilla segments between first and second floret 1-1.3(-1.6) $\mathrm{mm}$ long, with hairs (2.3-)3-3.6(-4.2) $\mathrm{mm}$ long; rachilla segments to sterile floret (0.6-)1.4-1.8(-2.3) mm long, with hairs (1.3-)1.7-2.3(-2.7) $\mathrm{mm}$ long. Lemmas (4.7-)5.6-6.1(-6.5) mm long $\times(0.8-) 1-1.4(-1.8) \mathrm{mm}$ wide, elliptic to oblong or broadly lanceolate, sometimes narrowly elliptic or narrowly lanceolate (ratio lemma width/lemma length $=(0.18-) 0.23-0.28(-0.33))$, scabridulous, sometimes glabrous, with hairs up to $0.1(-0.3) \mathrm{mm}$ long on the apex and margins, greenish, sometimes yellowish, surrounded by purple; apical teeth (0.1-)0.3-0.5(-0.9) $\mathrm{mm}$ long, with aristules (0.1-)0.4-0.6(-1) mm long; awn (5-)5.6-6.8(-7.8) $\mathrm{mm}$ long, inserted (2-)2.5-3.1(-3.3) $\mathrm{mm}$ from the base (ratio awn insertion from the base length/lemma length $=(0.36-) 0.43-$ $0.51(-0.53))$, geniculate near the base, rarely straight, not or slightly recurved to twisted, with adpressed hairs up to $0.1 \mathrm{~mm}$ long; callus $0.2-0.4 \mathrm{~mm}$ long, elliptic, sometimes oblong, with hairs (0.5-)1.2-2(-2.3) mm long. Paleas (4-)4.4-5.3(-6) $\mathrm{mm}$ long $\times(0.8-) 1-1.4 \mathrm{~mm}$ wide (ratio palea length/lemma length $=(0.7-) 0.78-0.91(-0.94))$, elliptic, sometimes slightly oblanceolate, rarely narrowly elliptic or oblong, with hairs on the upper part of the outer edges; keels with short hairs from the middle to the upper part, ending in teeth $0.2-0.4(-0.5) \mathrm{mm}$ long. Lodicules (0.4-)0.6-0.8(-1) mm long. Anthers (1.6-)2-2.5(-2.7) mm long. Ovary $0.5-0.7(-1) \mathrm{mm}$ long, glabrous, sometimes with hairs $0.1-0.4 \mathrm{~mm}$ long on the apex. Caryopsis 1.8-2.2 $\mathrm{mm}$ long $\times$ 0.4-0.6 mm wide.

a. subsp. BUSCHIANUM

T. rigidum var. molle Somm. \& Levier, Trudy Imp. S.Peterburgsk. Bot. Sada 16: 444. 1900.-TYPE: RUSSIA. Karachayevo-Cherkesiya, monte Elbrus, flumen Kükürtli, $43^{\circ} 21^{\prime} \mathrm{N}, 42^{\circ} 22^{\prime} \mathrm{E}, 10$ Sep 1890 , C.P.S. Sommier E E. Levier 1359 (lectotype, here designated, FI!; isolectotypes, G442508!, LE!).

T. transcaucasicum var. ciliatifolium Seredin, Bot. Mater. Gerb. Bot. Inst. Komarova Akad. Nauk S.S.S.R. 21: 55. 1961.TYPE: RUSSIA. Digorsky District, Karagom river, $43^{\circ} 9^{\prime} \mathrm{N}$, 4339’E, 11 Aug 1925, E. Busch E N. Busch 37 (holotype, LE!).

Herbs (17-)23.7-30.7(-35) cm tall, culm diam 0.6-0.9(-1.2) mm; nodes $4-7$, separated along the culm, mostly not enclosed by the sheaths. Basal leaf sheaths pubescent to densely pubescent, with hairs 0.4-08(-1) mm long; basal leaf blades $(1.3-) 2.7-3.4(-4.7) \mathrm{cm}$ long $\times 2.3-3.3(-4.7) \mathrm{mm}$ wide, flat, sometimes convolute when dried, pubescent to densely pubescent adaxially and abaxially, margins with hairs $0.6-1(-1.5) \mathrm{mm}$ long; top culm leaf sheaths (6-)6.7-9(-10) cm long, shorter than the internodes; top culm leaf blades (1.8-)2.5-5.7(-8) $\mathrm{cm}$ long $\times$ (2.5-)3-4.1(-5.2) mm wide, parallel to the culm, sometimes enclosing it or reaching to the panicle, glabrous to slightly pubescent adaxially and abaxially, with hairs (0.3-)0.7-1.1(-1.3) $\mathrm{mm}$ long, also on the margins; ligules (1.4-)1.6-2.1(-2.7) mm long, laciniate, rarely denticulate, glabrous, puberulous on the apex and rarely on the surface with hairs $0.2-0.6(-1.2) \mathrm{mm}$ long. Panicles (4.2-)5.2-7.4(-8.6) cm long $\times 1.3-2.6(-3.4) \mathrm{cm}$ wide, oblong to elliptic in outline, slightly dense; longest basal branches (0.6-)0.7-1.3(-1.7) cm. Lower glume acuminate, sometimes acute, 1(2-3)-nerved, greenish surrounded by purplish on the laterals; upper glume acuminate, sometimes acute, greenish surrounded by purplish. Lemma apical teeth (0.3-) 0.4-0.6(-0.9) mm long, with aristules (0.1-)0.3-0.6(-0.7) $\mathrm{mm}$ long; awn 5-6(-7.2) mm long, slightly or not recurved and more or less geniculate near the base, rarely straight; callus with hairs (0.5-) $1-1.8(-2) \mathrm{mm}$ long. Lodicules with apex bilobate. Anthers (2-) 2.3-2.7 mm long. Figure 9.

Chromosome Number-Unknown.

Phenology_Flowering and fruiting from June to September.

Distribution and Habitat-Endemic to West and Central Caucasus; at 1,980-2,900 m elevation; on stony slopes and moraines. Figure 10.

Specimens Examined-GEORGIA. Mtskheta-Mtianeti: Pirikiti Khevsureti, v. Arkhoti Netkhecho, $42^{\circ} 35^{\prime} \mathrm{N}, 44^{\circ} 51^{\prime} \mathrm{E}, 19$ Jul 1938, Grigoraschvli s. n. (MA); Kasbek, 17 Sep, Meyer 60 (LE); GroBer Kaukasus, Talweitung des T'ruso-Tales, westlich der Schluchtstrecke, 2.7-3.4 km W bis WNW Zemo Ok'rokana oder 7,2-7,9 km WNW Kobi, 42³5’N, 44²5’E, 23 Jul 1997, Schönswetter s. $n$. \& Tribsch (WU).

RUSSIA. Kabardino-Balkar Republic: Sukan River, between Aylangentash and Sukan-bashi-tsyfi natural landmarks, 27 Jul 1931, Bush s. n. \& Bush (LE); Sukan, 30 Jun 1927, Bush s. n. \& Bush (LE); Sukan, Altulany-bau-bashi, south slope between Ogary-vtsek and Kysyrtsy-kaya, 23 Jul 1931, Bush s. $n$. \& Bush (LE); upwards of Mystilyar-agach, 5 Jul 1913, Bush s. n. \& Bush (LE); adjoining Ullu-Guluku plane, $43^{\circ} 4^{\prime} \mathrm{N}, 43^{\circ} 15^{\prime} \mathrm{E}$, $10 \mathrm{Jul} 1913$, Bush s. $n$. \& Bush 


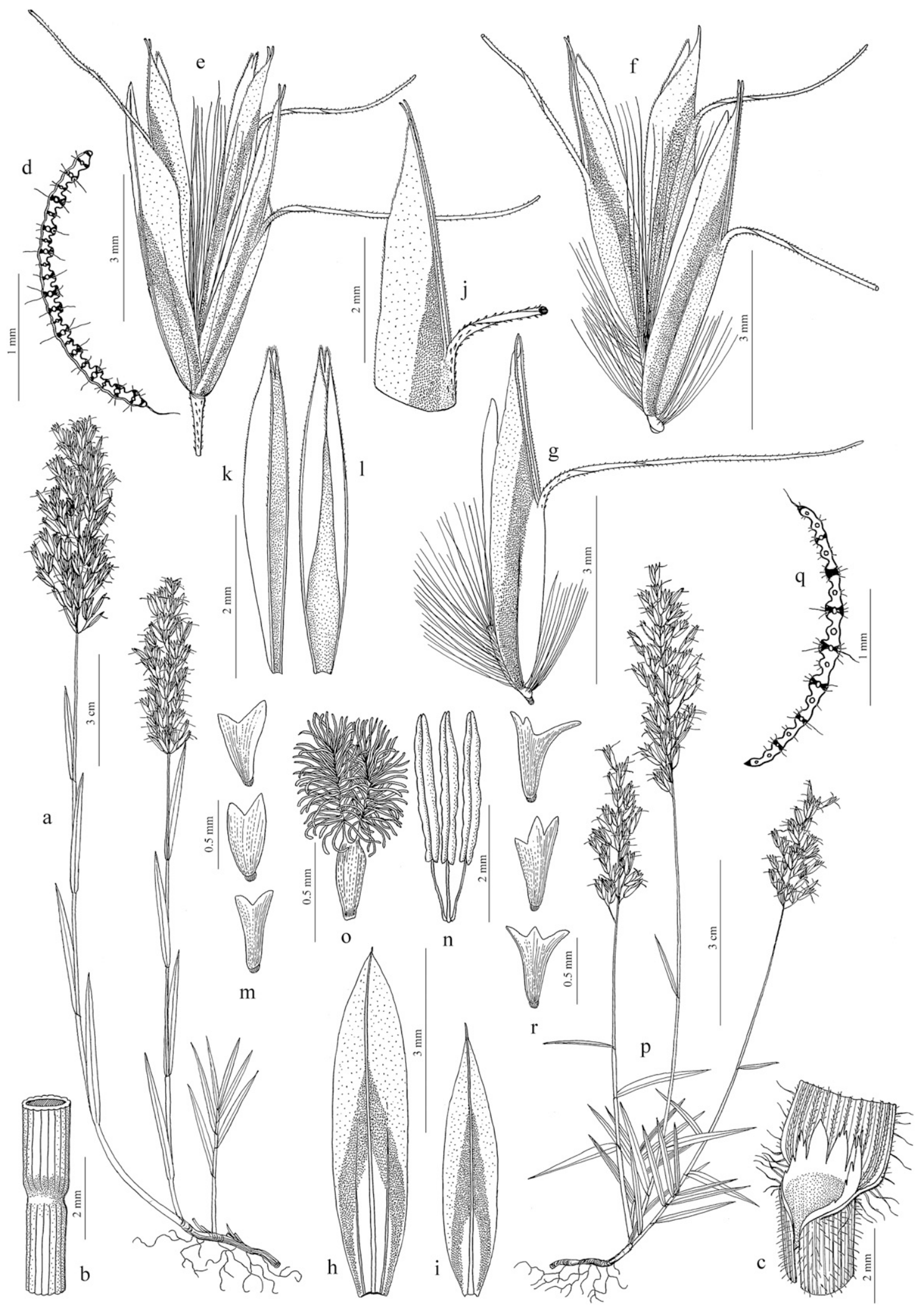

Fig. 9. A-O. Trisetum buschianum subsp. buschianum. A. Habit. B. Portions of the culm and node. C. Sheath, ligule, and portion of the blade. D. Transverse section of leaf blade. E. Spikelet. F. Florets. G. Floret. H. First glume, dorsal view. I. Second glume, dorsal view. J. Lemma, upper part, lateral view. K. Palea, dorsal view. L. Palea, ventral view. M. Lodicules. N. Stamens. O. Pistil. P-R. T. buschianum subsp. transcaucasicum. P. Habit. Q. Transverse section of leaf blade. R. Lodicules. (Lipsky s. n., LE, A; Wisniewski s. n., K, B-O; Bush \& Bush 11, Meyer s. n., LE, M; Karjagin s. n., HBG, P-R; Meyer 59, LE, R).

(LE); Shaurtu glacier, $43^{\circ} 7^{\prime} \mathrm{N}, 43^{\circ} 1^{\prime} \mathrm{E}, 25 \mathrm{Jul} 1913$, Bush s. $n$. \& Bush (LE); Bezengi glacier, $43^{\circ} 6^{\prime} \mathrm{N}, 43^{\circ} 9^{\prime} \mathrm{E}, 5$ Aug 1913, Bush s. n. \& Bush (LE); Bezengi glacier, $43^{\circ} 6^{\prime} \mathrm{N}, 43^{\circ} 9^{\prime} \mathrm{E}, 14$ Aug 1911, Bush s. $n$. \& Bush (LE); lower edge of Bezengi glacier, $43^{\circ} 6^{\prime} \mathrm{N}, 43^{\circ} 9^{\prime} \mathrm{E}$, 4 Jul 1913, Bush s. $n$. \& Bush (LE); Bezengi glacier, on the left bank of the Bezengi River, $43^{\circ} 6^{\prime} \mathrm{N}, 43^{\circ} 9^{\prime} \mathrm{E}, 6 \mathrm{Jul} 1913$, Bush s. n. \& Bush (LE); by Bezengi glacier, $43^{\circ} 6^{\prime} \mathrm{N}, 43^{\circ} 9^{\prime} \mathrm{E}, 25 \mathrm{Jul} 1892$, Bush s. $n$. \& Bush (LE); Sukan, moraines of Sukan-bashi-ullu-chiran glacier, 30 Jun 1927, Bush s. n. \& Bush (LE); by the left side of Mizhirgi glacier, $43^{\circ} 3^{\prime} \mathrm{N}, 43^{\circ} 10^{\prime} \mathrm{E}$, 


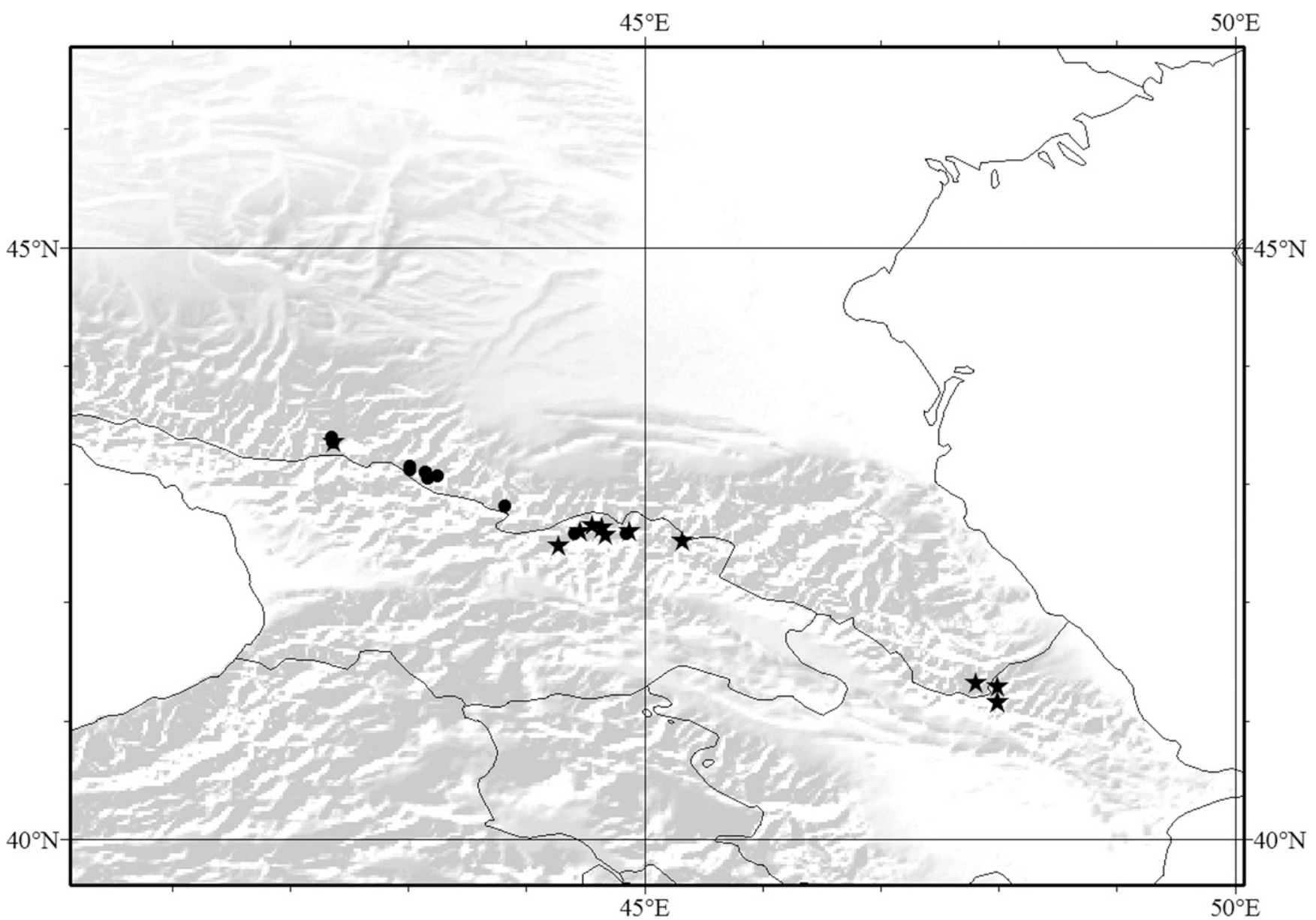

FIg. 10. Distribution of Trisetum buschianum subsp. buschianum (dots) and T. buschianum subsp. transcaucasicum (stars).

8 Jul 1913, Bush s. n. \& Bush (LE); Kulak glacier, upwards of Chegem, $43^{\circ} 9^{\prime} \mathrm{N}, 43^{\circ} 1^{\prime} \mathrm{E}, 27 \mathrm{Jul} 1913$, Bush s. $n$. \& Bush (LE); Mizhirgi glacier, $43^{\circ} 3^{\prime} \mathrm{N}$, $43^{\circ} 10^{\prime} \mathrm{E}, 16$ Aug 1911, Bush s. n. \& Bush (LE). Karachay-Cherkess Republic: glacier Kukurtlu, $43^{\circ} 21^{\prime} \mathrm{N}, 42^{\circ} 22^{\prime} \mathrm{E}, 13$ Aug 1961, Macha s. n. (MW); western foothills of Mount Elbrus, River basin Bitik-tyubyu, near the mineral springs, $43^{\circ} 24^{\prime} \mathrm{N}, 42^{\circ} 21^{\prime} \mathrm{E}, 31 \mathrm{Jul} 2008$, Zernov 6934 (MW); Karachai district, River Gorge Kukurtlu, left side, about $200 \mathrm{~m}$ from the edge Pednika, $43^{\circ} 21^{\prime} \mathrm{N}, 42^{\circ} 22^{\prime} \mathrm{E}, 3$ Aug 2009, Zernov 7263 (MW). North Ossetia: Digoria, the Sanguti glacier, $42^{\circ} 49^{\prime} \mathrm{N}, 43^{\circ} 49^{\prime} \mathrm{E}, 12$ Jul 1914, Gladkov 22 (LE).

b. subsp. TRANSCAUCASICUM (Seredin) Mosul., Zametki Sist. Geogr. Rast. 42: 34. 1991. Trisetum transcaucasicum Seredin, Bot. Mater. Gerb. Bot. Inst. Komarova Akad. Nauk S.S.S.R. 21: 54. 1961.-TYPE: AZERBAIJAN. Quba Dis-

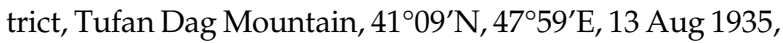
J. Karjagin s. n. (holotype: LE!; isotypes: GH!, MW!).

T. buschianum var. kasbeghi Seredin, Bot. Mater. Gerb. Bot. Inst. Komarova Akad. Nauk S.S.S.R. 21: 53. 1961.-TYPE: GEORGIA. Mount Kazbek, near Devdorak glacier, $42^{\circ} 41^{\prime} \mathrm{N}, 44^{\circ} 31^{\prime} \mathrm{E}, 3 \mathrm{Jul} 1916$, P. Krylov \& E. Steinberg s. $n$. (holotype: LE!).

T. rigidum var. ovale Somm. \& Levier, Trudy Imp. S.-Peterburgsk. Bot. Sada 16: 444. 1900.-TYPE: RUSSIA. Karachayevo-Cherkesiya, monte Elbrus, flumen Kükürtli, $43^{\circ} 20^{\prime} \mathrm{N}, 42^{\circ} 23^{\prime} \mathrm{E}, 10$ Sep 1890, C.P.S. Sommier \& E. Levier 1359 (lectotype, here designated, FI!; isolectotype: LE!).

Herbs (10.6-)13.6-18.6(-22.6) cm tall, culm diam 0.4-0.7 mm; nodes (3-)4-5(-8), concentrated in lower part of the culm, enclosed by the sheaths. Basal leaf sheaths densely pubescent with hairs $0.3-0.5 \mathrm{~mm}$ long, rarely glabrous; basal leaf blades (1-)2.3-3(-3.4) cm long $\times(1.9-) 2.1-2.9(-3.8) \mathrm{mm}$ wide, flat, sometimes inrolled when dried, pubescent to densely pubescent abaxially and adaxially, margins with hairs (0.1-)0.2-0.6(-0.7) $\mathrm{mm}$ long, greyish to greenish; top culm leaf sheaths (3-)3.6-6.2(-7.3) cm long, longer than the internodes, sometimes shorter; top culm leafblades (1.3)1.5-2.1(2.6) cm long $\times(-1.9) 2.1-2.7(-3.4) \mathrm{mm}$ wide, sometimes conduplicate, perpendicular or parallel to the culm, glabrous to slightly pubescent abaxially and adaxially, margins with hairs (0.05-)0.1-0.6(-1.3) mm long; ligules (1.2-)1.7-2(-3) mm long, laciniate, glabrous, rarely with few hairs up to $0.3 \mathrm{~mm}$ on the apex. Panicle (3.4-)3.6-4.6(-7.1) cm long $\times(1.6-) 1.8-2.5 \mathrm{~cm}$ wide, ovate to elliptic in outline, rarely lanceolate, lax to slightly dense; longest basal branches (0.5-)0.8-1.2(-2.6) cm. Lower glume acuminate, sometimes long acuminate or acute, 1(2)-nerved, dark purple to purplish, rarely greenish, laterals and upper part hyaline or yellowish; upper glume acuminate, sometimes long acuminate, rarely acute, greenish or purplish on the central part, laterals hyaline or yellowish. Lemma apical teeth (0.1-)0.2-0.4(-0.5) mm long, with aristules (0.2-)0.5-0.8(-1) mm long; awn (5.4-)6-7.3 $(-7.8) \mathrm{mm}$ long, geniculate and twisted near the base; callus with hairs (1.2-)1.6-2.2(-2.3) mm long. Lodicules apex irregular or regular 2-4-lobed, sometimes with one of them lateral. Anthers (1.6-)2-2.3(-2.6) mm long. Figure 9.

Chromosome Number-Unknown.

Phenology_Flowering and fruiting from July to September. 
Distribution and Habitat-Endemic to the Central and Eastern part of the Caucasus; at 1,740-3,250 m elevation; in alluvial rocks and schists. Figure 10.

Specimens Examined-AZERBAIJAN. Baku: Kuba district, mons Tufan-Dag, 41 ${ }^{\circ} 9^{\prime} \mathrm{N}, 47^{\circ} 59^{\prime} \mathrm{E}, 6$ Aug 1935, Karjagin s. n. (B, GH, HBG); 6 Aug 1935, Karjagin s. n. (HBG); 31 Jul 1830, Meyer s. n. (LE); in m. Shach-Dagh, Buldurgan, $41^{\circ} 17^{\prime} \mathrm{N}, 47^{\circ} 59^{\prime} \mathrm{E}, 23$ Aug 1929, Sachokjia s. n. (LE).

GEORGIA. Kakheti: Großer Kaukasus, Zentralteil, Tush-PsavKhevsureti, Omalo, im Bereich der Quellzuflüsse des Pirikita Alazani östlich des Azunta-Passes, $42^{\circ} 31^{\prime} \mathrm{N}, 4^{\circ} 19^{\prime} \mathrm{E}, 8$ Sep 2009, Zündorf 25658 \& Gerth (JE). Mtskheta-Mtianeti: Ermani area, Fidar Volcano, $42^{\circ} 29^{\prime} \mathrm{N}$ $44^{\circ} 16^{\prime}$ E, 22 Aug 1940, Dolukhanov s. n. \& Mandenova (LE); Greater Caucasus,

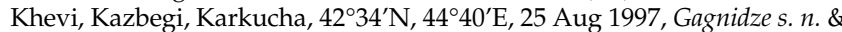
al. (W); Pirikiti Khevsureti, gorge Arhiele, bottom glacier Kidegana, $42^{\circ} 36^{\prime} \mathrm{N}, 44^{\circ} 52^{\prime} \mathrm{E}$, Jul 1937, Grigoraschvli s. n. (MA); Großer Kaukasus, linksseitige Alluvionen des Flusses Terek zwischen Ach'khoti, P'anshet' und Kazbegi, 42 $2^{\circ} 38^{\prime} \mathrm{N}, 4^{\circ} 38^{\prime} \mathrm{E}, 23 \mathrm{Jul}$ 1997, Schönswetter s. n. \& Tribsch (WU); Schneeweiß s. n. (WU); Großer Kaukasus T'ruso-Schlucht 0.5-2.7 km WNW Zemo Ok'rokana, d.i. ca. 5-7.2 km WNW Kobi, 42 ${ }^{\circ} 36^{\prime} \mathrm{N}, 44^{\circ} 27^{\prime} \mathrm{E}, 23$ Jul 1997, Schönswetter s. n. \& Tribsch (WU); Großer Kaukasus, Zentralteil, Kasbek-(Mkinvarzweri) Massiv westlich Kasbegi, Aufstieg über die Tsminda Sameba, am Fuß des SO-Gletschers am Kasbek, 42 $39^{\prime} \mathrm{N}, 44^{\circ} 33^{\prime} \mathrm{E}$, 6 Aug 2007, Zündorf 24247 (JE).

RUSSIA. Dagestan: Dokuzparinsky district, Gora Shalbuzdag, $41^{\circ} 19^{\prime} \mathrm{N}$, $47^{\circ} 48^{\prime}$ E, 25 Sep 2006, Kotseruba 56 (LE).

Notes-Trisetum buschianum is close to T. rigidum, having smaller panicles, usually dark purple, with lower and upper glumes similar in length, longer callus hairs and smaller ligules. Trisetum rigidum also has longer hairs on the rachilla segments between the first and second floret than Trisetum buschianum ((3.2-)4-4.7(-5.7) and (2.3-)3-3.6(-4.2) $\mathrm{mm}$ long, respectively) and longer lemmas ((5.3-)6.4-8.2(-9.3) and 5.6-6.2 mm long, respectively).

We have accepted the criterion of Mosulishvili (1991), and recognize $T$. buschianum and $T$. transcaucasicum at the subspecific level. Tzvelev (1976) commented about the closeness of these species and hinted that perhaps they should be treated as a subspecies. According to Du Riezt (1930), the subspecies of a species are commonly reciprocally intergrading, and their taxonomical delimitation is thus infinitely more arbitrary than that of the species; Stuessy et al. (2014) recognized geography as a fundamental component for recognition of infraspecific taxa. Trisetum buschianum subsp. buschianum lives in the central Caucasus, and T. buschianum subsp. transcaucasicum in the central and eastern parts. These two subspecies overlap only in the central part, where there are transitional taxa such as T. buschianum var. kasbeghi and T. transcaucasicum var. ciliatifolium. Trisetum buschianum subsp. transcaucasicum is smaller and has shorter top culm leaf blades than those of subsp. buschianum. The disposition of leaves is normally parallel to the culm and sometimes the leaves reach the panicle in subsp. buschianum, whereas the basal leaves are always perpendicular in subsp. transcaucasicum. Trisetum buschianum subsp. transcaucasicum has ovate to elliptic panicles, which are oblong to elliptic in subsp. buschianum.

6. Trisetum velutinum Boiss., Elench. Pl. Nov.: 88. 1838. Avena velutina (Boiss.) Nyman, Consp. Fl. Eur.: 812. 1882. Trisetaria velutina (Boiss.) Paunero, Anales Jard. Bot. Madrid 9: 512. 1950.-TYPE: SPAIN. Granada, Sierra Nevada, 3703N, 329' W, Aug 1837, E. Boissier 187 (lectotype, designated by Burdet et al. (1981: 580), G-176291!; isolectotypes: B-100250154!, G-176292!, G-176293!, G176294!, G-176295!, G-176296!, G-176297!, G-439749!, GH!, GOET-6971!, H!, JE-18835!, K-808725!, K-808726!, LE!, M-210840!, MA-8011!, NY!, P-2222135!, P-2222141!, P-2222145!, P-2222149!, S-12-12490!, S-13-33131!, SI-30137!, W-1889-0242141!).
T. cavanillesianum Borja \& Font Quer, Anales Jard. Bot. Madrid 6: 494. 1946. Trisetum velutinum subsp. cavanillesianum (Borja \& Font Quer) O. Bolòs \& Vigo, Fl. Països Catalans 4: 476. 2001.-TYPE: SPAIN. Valencia, sierra de la Murta, cumbres del "Cavall Vernal", 39॰9'N, $0^{\circ} 21^{\prime} \mathrm{W}$, J. Borja s. $n$. (lectotype, here designated, MA-8010!).

Herbs (16-)25.5-51(-68) cm tall, not or loosely tufted, shortly rhizomatous or with stoloniferous rhizomes, culms (0.25-)0.4-0.8(-1) mm diam, glabrous, rarely slightly puberulous on the upper part, with hairs $0.1 \mathrm{~mm}$ long; nodes (4-)6.5-10(-11), more or less concentrated in lower part of the culm, enclosed by the sheaths except the upper ones, slightly puberulous or glabrous. Basal leaf sheaths densely pubescent, with hairs (0.3-)0.8-1.2(-1.5) mm long, greyish to greenish or yellowish to brownish; basal leaf blades (2.8-)3.3-6.6(-7.4) cm long $\times(1.3-) 2.3-4(-4.8) \mathrm{mm}$ wide, flat to slightly inrolled when dried, densely pubescent abaxially and adaxially, usually longer adaxially, with hairs $0.1-1(-1.2) \mathrm{mm}$ long, greyish to greenish; top culm leaf sheaths (4.8-)7.2-14(-17) cm long, shorter than the internodes, sometimes longer, glabrous to puberulous, with adpressed hairs, normally with cilia along the margins; top culm leaf blades (0.3-)1.5-3.4(-4) cm long $\times$ (1-)1.2-2(-2.2) mm wide, inrolled along the margins, sometimes parallel to the culm, densely pubescent abaxially, from glabrous to densely pubescent adaxially, with hairs up to $0.2 \mathrm{~mm}$ long, greenish to greyish; inner collar region glabrous or with the same indumentum as the leaf blade, sometimes with hairs $0.1(-0.2) \mathrm{mm}$ long along the margins; ligules (0.5-)1.4-2(-2.5) mm long, laciniate to slightly laciniate, slightly pubescent to pubescent, usually densely on the apex, with hairs (0.05-)0.1-0.4(-0.6) $\mathrm{mm}$ long. Basal node of the panicle glabrous or very slightly puberulous, with hairs less than $0.1 \mathrm{~mm}$ long. Panicles (4-)5.9-10.5(-15) cm long $\times(0.7-) 1-$ 2.6(-3.6) $\mathrm{cm}$ wide, narrowly elliptic to narrowly or broadly lanceolate in outline, sometimes linear, lax to slightly dense with most rachis internodes plainly visible; rachis glabrous to slightly pubescent, with hairs up to $0.1 \mathrm{~mm}$ long; longest basal branches (0.4-)0.5-1.4(-3) cm. Spikelets (5-)6.4-8.5 mm long $\times$ (1-)2-2.6(-3.2) mm wide, 2(3)-flowered; pedicels (1.7-)2.3$4(-6.5) \mathrm{mm}$ long, pubescent to slightly pubescent, rarely glabrous, with hairs up to $0.1 \mathrm{~mm}$ long. Glumes unequal to subequal (ratio lower glume length/upper glume length $=(0.71-) 0.78$ $0.8(-0.85)$ ); lower glume (3.5-)4.6-5.6(-6.2) mm long $\times(0.7-) 1-$ $1.1(-1.4) \mathrm{mm}$ wide, narrowly lanceolate, sometimes broadly lanceolate, rarely narrowly elliptic (ratio lower glume width/ lower glume length $=(0.11-) 0.17-0.23(-0.29))$, acuminate, sometimes long acuminate, 1(3)-nerved, glabrous, with very short hairs on the midrib up to $0.08 \mathrm{~mm}$ long, sometimes on the upper part of the margins, greenish to yellowish on the central part, laterals and upper part hyaline; upper glume (4-)5.77.2(-8) mm long $\times(1.4-) 1.6-1.7(-2) \mathrm{mm}$ wide, oblong to lanceolate or narrowly elliptic to narrowly lanceolate, rarely slightly oblanceolate or elliptic (ratio upper glume width/upper glume length $=(0.2-) 0.25-0.28(-0.34))$, acuminate, usually acuminate, 3-nerved, glabrous, with very short hairs up to $0.05 \mathrm{~mm}$ long from the middle to the upper part of the central nerve and upper part of the margins, greenish to yellowish on the central basal part, usually also the central nerve, laterals and upper part hyaline or yellowish; rachilla segments between first and second floret 1-1.8 mm long, with hairs (0.3-)0.7-1.6(-2.4) mm long; rachilla segments to sterile floret (0.8-)1.7-2.4(-3) $\mathrm{mm}$ long, with 
hairs (0.3-)0.6-1(-1.5) mm long. Lemmas (4-)5.4-6.6(-7.8) mm long $\times(0.7-) 1-1.1(-1.4) \mathrm{mm}$ wide, narrowly elliptic or elliptic to narrowly lanceolate, rarely oblong or slightly oblanceolate (ratio lemma width/lemma length $=(0.14-) 0.19-0.27(-0.37))$, glabrous to strigulose, yellowish to greenish; apical teeth $0.2-0.4(-0.9) \mathrm{mm}$ long, with aristules (0-)0.3-0.8(-2.5) mm long; awn (5-)6-7.6 (-9.3) $\mathrm{mm}$ long, inserted (1.3-)2-2.6(-5) $\mathrm{mm}$ from the base (ratio awn insertion from the base length/lemma length $=(0.25-)$ $0.34-0.43(-0.64))$, slightly twisted and more or less geniculate near the base, rarely straight, with very short adpressed hairs $0.03 \mathrm{~mm}$ long; callus (0.1-)0.2-0.3(-0.4) mm long, elliptic to orbicular or oblong, with hairs $0.25-0.5(-0.7) \mathrm{mm}$ long. Paleas (2.4-) 4.2-5.1(-6.1) $\mathrm{mm}$ long $\times(0.6-) 0.75-1.5 \mathrm{~mm}$ wide (ratio palea length/lemma length $=(0.36-) 0.76-0.83(-0.89))$, narrowly elliptic, sometimes elliptic, slightly hairy on the upper part of the outer edges; keels with short hairs mostly on the apex, ending in teeth (0.1-)0.3-0.4 mm long. Lodicules (0.4-)0.5-1(-1.6) $\mathrm{mm}$ long, with apex irregularly dentate or 2-lobed, rarely with scattered hairs. Anthers (1.6-)2-3.1(-4) mm long. Ovary (0.4-)0.6-1.2 $(-1.5) \mathrm{mm}$ long, glabrous, rarely with some hairs on the apex up to $0.2 \mathrm{~mm}$ long. Caryopsis $1.9-2 \mathrm{~mm}$ long $\times 0.5-0.6 \mathrm{~mm}$ wide. Figure 11.

Chromosome Number $-n=14$ (Devesa \& Romero Zarco 1984).

Phenology-Flowering from June to August.

Distribution and Habitat-Endemic to southeastern and eastern Spain; at 400-2,280 m elevation; in fissures of limestones and dolomitic sand soils, in open places. Figure 3.

Specimens Examined-SPAIN: Albacete: Ayna, mirador sobre la piscina, $38^{\circ} 33^{\prime} \mathrm{N}, 2^{\circ} 3^{\prime} \mathrm{W}$, 20 Jul 1984, Luceño s. n. (MA); barrancos del río Mundo, Ayna, 38 $33^{\circ} \mathrm{N}, 2^{\circ} 4^{\prime} \mathrm{W}, 11 \mathrm{Jul} 1971$, Rivas Goday s. $n$. $\mathcal{E}$ al. (VAL). Alicante: Quatretonda, Serra de Serrella, Les Frares, $38^{\circ} 42^{\prime} \mathrm{N}, 0^{\circ} 17^{\prime} \mathrm{W}, 30$ Jun 1997, Solanas s. $n$. E al. (ABH); Sierra de la Safor, Vall de Gallinera, $38^{\circ} 51^{\prime} \mathrm{N}, 0^{\circ} 14^{\prime} \mathrm{W}$, 15 Jun 1997, Soler 7265JXS \& Signes (MA); Castell de Castells (Marina Alta), serra d'Alfaro, 3844'N, 0¹3’W, 7 Jul 1997, Soler JXS-7339 \& Signes (VAL). Granada: Sierra Nevada, Monte Dornajo, $37^{\circ} 7^{\prime} \mathrm{N}$, 326'W, 15 Jul 1851, Bourgeau 1519 (G, GOET, K, LE, P, S); Jul 1891, Porta 648 E Rigo (B, FI, G, JE, K, M, MA, NY, P, S, W, WU); 30 Sep 1975, Fernández Casas 963B (MA); ad Dornajo et Tesoro, $37^{\circ} 26^{\prime} \mathrm{N}, 3^{\circ} 37^{\prime} \mathrm{W}$, Jul 1879, Huter 1171 \& al. (B, K, GB, L, LE, M, MPU, P, PR, WU); Sierra Nevada, pico del Tesoro, 37 ${ }^{\circ} 5^{\prime} \mathrm{N}, 3^{\circ} 27^{\prime} \mathrm{W}, 14$ Aug 2011, Aedo 18599 (MA); Sierra Nevada, pico Trevenque, $37^{\circ} 4^{\prime} \mathrm{N}, 3^{\circ} 28^{\prime} \mathrm{W}, 13 \mathrm{Jul} 1986$, Aedo s. n. (MA); $37^{\circ} 4^{\prime} \mathrm{N}, 3^{\circ} 28^{\prime} \mathrm{W}, 25$ Jul 2013, Barberá 941 \& al. (MA); Barberá 942 \& al. (MA); VII, Del Campo 93 (GB, GH, H, LE, M, MA, MPU, O, P, S, W); 16 Jun 1852, Del Campo 98 (B, L, MPU, P); 11 Jul 1990, Wagenitz 4707 \& Hellwig (GOET, JE); Trevenque, Canal de Huenes, $37^{\circ} 5^{\prime} \mathrm{N}, 3^{\circ} 31^{\prime} \mathrm{W}, 20 \mathrm{Jul} 1974$, Charpin s. n. E Fernández Casas (B, C, M, MA, NY); Sierra Nevada, Alayos de Dilar, Corazón de la

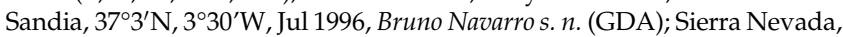
Barranco de las Víboras, $37^{\circ} 7^{\prime} \mathrm{N}, 3^{\circ} 27^{\prime} \mathrm{W}$, 16 Jul 1981, Romero C170/81 (SEV); Sierra Nevada, Hoya de la Mora, $37^{\circ} 5^{\prime} \mathrm{N}, 3^{\circ} 22^{\prime} \mathrm{W}, 28 \mathrm{Jul}$ 1967, Segura Zubizarreta s. n. (MA); Sierra de Alfacar, $37^{\circ} 14^{\prime} \mathrm{N}$, $3^{\circ} 33^{\prime} \mathrm{W}$, 2 Jul 1878, Hegelmaier s. n. (JE, P); Sierra de Jatar, $36^{\circ} 56^{\prime} \mathrm{N}$, 3⒌' $\mathrm{W}, 13$ Jul 1977, Litzler 77/765E (MPU); Sierra Almijara, cerro del Lucero, 36 $6^{\circ} 52^{\prime} \mathrm{N}, 3^{\circ} 53^{\prime} \mathrm{W}, 7$ Aug

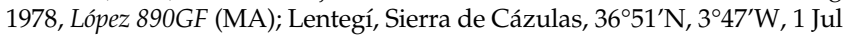
1978, Martínez Parras s. n. (MA); carretera de Murcia a $3 \mathrm{~km}$ del Puerto de la Mora, $37^{\circ} 16^{\prime} \mathrm{N}, 3^{\circ} 27^{\prime} \mathrm{W}$, Jun 1986 , Morales s. $n$. (GDA); Padul, camino de los Gudaris, $37^{\circ} 2^{\prime} \mathrm{N}, 3^{\circ} 34^{\prime} \mathrm{W}, 5 \mathrm{Jul}$ 2000, Morales s. n. E Baena (GDA); Sierra del Manar, $37^{\circ} 2^{\prime} \mathrm{N}, 3^{\circ} 36^{\prime} \mathrm{W}$, 18 Jun 1980, Romero s. n. \& Morales (GDA); Sierra de Huetor, Pico Calabozo, $37^{\circ} 17^{\prime} \mathrm{N}, 3^{\circ} 26^{\prime} \mathrm{W}, 25 \mathrm{Jul} 1978$, Romero s. $n$. \& Valle (GDA); Cogollos de la Vega, proximidades a Cortijo de los Asperones, $37^{\circ} 18^{\prime} \mathrm{N}, 3^{\circ} 31^{\prime} \mathrm{W}, 17 \mathrm{Jul} 1988$, Socorro s. n. E al. (GDA). Málaga: Cómpeta, Sierra de Cómpeta, 3650’N, $3^{\circ} 56^{\prime} \mathrm{W}, 7$ Jul 1973, Cabezudo s. n. \& Valdés (SEV); 36 $50^{\circ} \mathrm{N}, 3^{\circ} 56^{\prime} \mathrm{W}, 7$ Jul 1973, Valdés s. $n$. E al. (MA); Sierra de la Tejeda, subida por Canillas del Aceituno, 36 ${ }^{\circ} 53^{\prime} \mathrm{N}, 4^{\circ} 3^{\prime} \mathrm{W}$, 19 Aug 1977, López s. n. (MA, SEV); 15 Jul 1981, Romero s. n. (B, BC, BCN, G, GDA, H, LE, MA, SEV, VAL, WU). Valencia: Serra de la Murta, Cavall Vernat, $39^{\circ} 9^{\prime} \mathrm{N}$, $0^{\circ} 21^{\prime} \mathrm{W}, 10 \mathrm{Jul} 1946$, Borja s. n. (ARAN, BC, BCN, F, MA, SEV); Quatretonda, Umbría del Buixcarró, $39^{\circ} 0^{\prime} \mathrm{N}, 0^{\circ} 21^{\prime} \mathrm{W}, 12$ Apr 1997, Herrero-Borgoñón s. n. (ABH, MA); Villalonga, Sierra de La Safor, $38^{\circ} 51^{\prime} \mathrm{N}, 0^{\circ} 15^{\prime} \mathrm{W}$, 11 May 1996, Herrero-Borgoñón s. n. E al. (ABH); Buñol, El Fustal, $39^{\circ} 25^{\prime} \mathrm{N}, 0^{\circ} 50^{\prime} \mathrm{W}, 11$ Jun
1994, Laguna s. n. (ABH); Buñol, Sierra de Malacara, umbría del Fresnal, $39^{\circ} 25^{\prime} \mathrm{N}, 0^{\circ} 38^{\prime} \mathrm{W}, 25 \mathrm{Jul} 1991$, Laguna s. n. (VAL); Fuente Umbría, Collado de Umán, Font de Roses, Buñol, 39²5’N, 056’W, 13 Aug 1990, Laguna s. n. E Crespo (VAL); Barig, $39^{\circ} 0^{\prime} \mathrm{N}, 0^{\circ} 18^{\prime} \mathrm{W}$, Jun 1980, Monsanet s. $n$. E Mateo (VAL); Quatretonda, La Vall d'Albaida, ombria del Buixcarró, $39^{\circ} 0^{\prime} \mathrm{N}$, $0^{\circ} 21^{\prime} \mathrm{W}$, Riera 3135 (VAL); Quatretonda (La Vall d'Albaida), els Cuderellets, 38 $59^{\prime} \mathrm{N}, 0^{\circ} 22^{\prime} \mathrm{W}, 26$ Jun 1996, Riera 3151 (VAL); Moixent (La Costera), pr. $1^{\prime}$ Heura, $38^{\circ} 53^{\prime} \mathrm{N}, 0^{\circ} 48^{\prime} \mathrm{W}$, $17 \mathrm{Jul} 1997$, Riera 3719 (VAL); Moixent (La Costera), bc. De la Falç, 3853’N $0^{\circ} 46^{\prime} \mathrm{W}$, 17 Jul 1997, Riera 3729 (VAL); Vilallonga de la Safor, Serra de la Safor, pr. Alt de la Safor, $38^{\circ} 51^{\prime} \mathrm{N}, 0^{\circ} 15^{\prime} \mathrm{W}$, 12 Jul 2001, Riera JRV-4480 \& Andrés (VAL); Simat de Valldigna, entre Les Foies y Mondúver, $39^{\circ} 1^{\prime} \mathrm{N} 0^{\circ} 17^{\prime} \mathrm{W}, 26$ Apr 2015, Barberá 1617 \& Quintanar (MA); Simat de Valldigna (La Safor), Les Foies, $39^{\circ} 1^{\prime} \mathrm{N}, 0^{\circ} 17^{\prime} \mathrm{W}, 2$ Aug 1990, Soriano 0917PS (VAL); Quatretonda, Pista Els Corrals-Pinet (La Vall $\mathrm{d}^{\prime}$ Albaida), 38 $59^{\prime} \mathrm{N}, 0^{\circ} 20^{\prime} \mathrm{W}$, $5 \mathrm{Jul}$ 1991, Soriano 1652PS (VAL).

Notes-This species is clearly distinguished because of its velutinous leaves. Font Quer (1946) published certain specimens from the eastern Iberian Peninsula as Trisetum cavanillesianum, without making any comparison with $T$. velutinum. The new species may have stood out for its smaller panicle and the general size of the plant, but T. velutinum is a highly variable species and we believe that $T$. cavanillesianum does not deserve any taxonomic recognition.

In Trisetum velutinum the formation of propagules in the inflorescence by pseudoviviparism has been observed (Pierce et al. 2003) in the specimen collected by Barberá 1617 \& Quintanar (MA). T. velutinum is one of the three species of the genus with pseudoviviparism; the others are the Canarian endemic T. tamonanteae Marrero Rodr. \& S. Scholz and T. rigidum. According to Marrero Rodríguez and Scholz (2013), this phenomenon becomes intensified and affects the whole inflorescence when the plants bloom in especially humid climatic conditions. This situation is common in the habitat of these species.

7. Trisetum tamonanteae Marrero Rodr. \& S. Scholz, Willdenowia 43: 47, 48 Fig. 1, 49 Fig. 2. 2013.-TYPE: SPAIN. Canary Islands, Fuerteventura, Pájara, Jandía, Pico de La Zarza, $28^{\circ} 06^{\prime} 02^{\prime \prime} \mathrm{N}, 14^{\circ} 21^{\prime} 27^{\prime \prime} \mathrm{W}, 11$ Jun 1987, A. Marrero s. n. (holotype: LPA-24804 (image!); isotype: B).

Herbs 19-30(-32) cm tall, densely tufted, shortly rhizomatous, culms (0.5-)0.6-0.7 $\mathrm{mm}$ diam, pubescent, with hairs $0.2-0.3(-0.5) \mathrm{mm}$ long; nodes 5-10, separated along the culm, enclosed by the sheaths, rarely exposed, glabrous, rarely with some hairs. Basal leaf sheaths densely pubescent, rarely the oldest ones glabrous, with hairs $0.4-0.8 \mathrm{~mm}$ long, yellowish to brownish, rarely greyish; basal leaf blades (3.2-)4.7-9.3(-12.2) $\mathrm{cm}$ long $\times(1.3-) 1.7-3 \mathrm{~mm}$ wide, scattered or distichous in juvenile culms, flat, sometimes inrolled when dried, pubescent abaxially and adaxially, margins with hairs $0.2-0.5 \mathrm{~mm}$ long, yellowish to brownish; top culm leaf sheaths (2.6-)3.8$5.7(-6.5) \mathrm{cm}$ long, longer than the internodes, sometimes shorter, pubescent to densely pubescent, without adpressed hairs and cilia along the margins; top culm leaf blades (2-)2.7$10(-14) \mathrm{cm}$ long $\times 2.8-4.5(-6) \mathrm{mm}$ wide, flat, usually reaching or surpassing the panicle, pubescent to densely pubescent abaxially and adaxially, with adpressed and not adpressed hairs, with hairs on the margins up to $0.3 \mathrm{~mm}$ long, greenish to greyish, sometimes brownish; inner collar region with the same indumentum as the leaf blade, with scattered hairs $0.5-1.3 \mathrm{~mm}$ long along the margins; ligules (0.8-)1-1.4 mm long, laciniate to slightly laciniate, glabrous. Basal node of the panicle glabrous. Panicles 3-7.7(9.7) cm long $\times(1.2) 1.4-1.7(-2)$ $\mathrm{cm}$ wide, narrowly to broadly lanceolate in outline, sometimes from narrowly elliptic to oblong, slightly dense, with most rachis internodes plainly visible; rachis densely pubescent, 


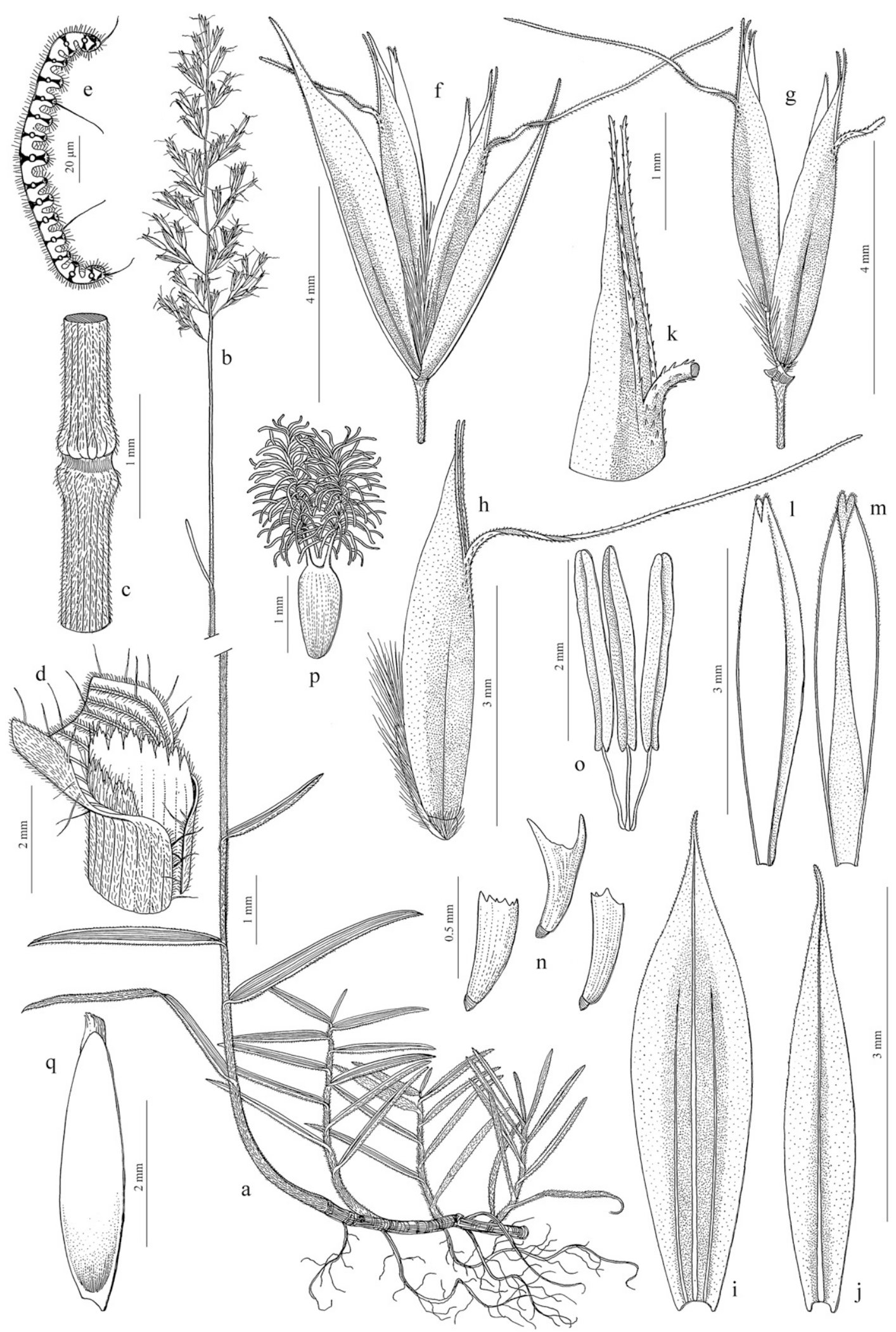

Fig. 11. Trisetum velutinum. A. Habit. B. Inflorescence. C. Portion of the culm and node. D. Sheath, ligule, and portion of the blade. E. Transverse section of leaf blade. F. Spikelet. G. Florets. H. Floret. I. First glume, dorsal view. J. Second glume, dorsal view. K. Lemma, upper part, lateral view. L. Palea, dorsal view. M. Palea, ventral view. N. Lodicules. O. Stamens. P. Pistil. Q. Caryopsis. (A-D, F-M, O-Q: Porta \& Rigo 648, B-100526421, MA-8015. E: Signes \& Soler 7339, VAL-218705. N: Cuatrecasas \& Font Quer s. n., BC-878863, Riera 3729, VAL-37841). 
sometimes pubescent, with hairs up to $0.6 \mathrm{~mm}$ long; longest basal branches (0.3-)0.5-1.4 cm. Spikelets 4.5-5.8 $\mathrm{mm}$ long $\times$ 2.7-4 mm wide, (2)3-5-flowered; pedicels (1-)1.7-2.4(-3.7) $\mathrm{mm}$ long, pubescent, with hairs up to $0.3 \mathrm{~mm}$ long. Glumes unequal to subequal (ratio lower glume length/upper glume length $=$ (0.75-)0.77-0.88(-0.91)); lower glume 3.2-4.5 $\mathrm{mm}$ long $\times$ 0.7-0.8(-1) $\mathrm{mm}$ wide, narrowly lanceolate to narrowly elliptic (ratio lower glume width/lower glume length $=0.18$ $0.22(-0.3)$ ), acute to acuminate, rarely long acuminate, $1(2)$ nerved, glabrous, with some hairs on the main nerve up to $0.2 \mathrm{~mm}$ long, greenish on the central part, sometimes yellowish, rest hyaline; upper glume 4-5.7(-6) $\mathrm{mm}$ long $\times$ 1.2-1.4 mm wide, oblanceolate, sometimes elliptic or lanceolate (ratio upper glume width/upper glume length = 0.2-0.33), acuminate, sometimes acute, 3-nerved, glabrous, with short hairs up to $0.2 \mathrm{~mm}$ long on the central nerve, sometimes also on the margins, greenish on the central part, the remaining hyaline; rachilla segments between first and second floret (0.3-)0.5-0.6 mm long, with hairs 0.1-0.3(-0.7) $\mathrm{mm}$ long; rachilla segments to sterile floret $0.4-0.6(-0.8) \mathrm{mm}$ long, with hairs $0.1-0.4 \mathrm{~mm}$ long. Lemmas (2.6-)3-4.3(-5.4) $\mathrm{mm}$ long $\times 0.7-0.8(-1) \mathrm{mm}$ wide, oblong to elliptic, sometimes oblanceolate (ratio lemma width/lemma length $=0.25$ $0.38(-0.41))$, strigulose, sometimes with short hairs on the aristules, longers on the midrib, up to $0.2 \mathrm{~mm}$ long, yellowish to greenish, sometimes brownish; apical teeth $0.2-0.5 \mathrm{~mm}$ long, with aristules $0.1-0.7(-0.9) \mathrm{mm}$ long, rarely without aristules; awn (2.8-)3.4-5.1(-6.1) mm long, inserted (0.9-)1.1-2.1 mm from the base (ratio awn insertion from the base length/lemma length $=(0.26-) 0.3-0.4(-0.53))$, straight, sometimes slightly twisted on the middle, with very short adpressed hairs $0.05 \mathrm{~mm}$ long; callus $0.1-0.2 \mathrm{~mm}$ long, elliptic to rounded, with hairs 0.1-0.3 mm long. Paleas $2.3-3 \mathrm{~mm}$ long $\times 0.6-0.8 \mathrm{~mm}$ wide (ratio palea length/lemma length $=(0.56-) 0.63-0.74)$, narrowly elliptic, sometimes oblanceolate, with short hairs along the outer edges; keels with short hairs, ending in teeth (0.1-)0.2 mm long. Lodicules (0.3-)0.4-0.6 mm long, with apex slightly bilobate, one of them lateral. Anthers $0.8-1.6(-1.8) \mathrm{mm}$ long. Ovary $0.3-0.7(-0.8) \mathrm{mm}$ long, glabrous. Caryopsis (1.6-)1.8-2(-2.2) mm long.

Chromosome Number-Unknown.

Phenology_Flowering from April to July.

Distribution and Habitat-Endemic to Jandía massif, Fuerteventura (Canary Islands); at 700-800 m elevation; in volcanic rock fissures and cracks. Figure 12.

Specimens Examined-SPAIN. Canary Is.: Fuerteventura, Pájara, Jandía, Pico de La Zarza, $28^{\circ} 6^{\prime} \mathrm{N}, 1^{\circ} 21^{\prime} \mathrm{W}, 30$ Mar 1989, Marrero s. n. (LPA, MA, P); $28^{\circ} 6^{\prime} \mathrm{N}, 14^{\circ} 21^{\prime} \mathrm{W}, 26$ Jul 2000, Marrero s. n. \& Scholz (LPA); $28^{\circ} 6^{\prime} \mathrm{N}$, $14^{\circ} 21^{\prime} \mathrm{W}, 8$ Apr 2000, Marrero s. $n$. $\mathcal{E}$ Scholz (LPA); entre el Pico de la Zarza y el Pico del Mocán, $28^{\circ} 6^{\prime} \mathrm{N}, 14^{\circ} 21^{\prime} \mathrm{W}$, Marrero s. n. E Scholz (LPA); 8 Apr 2000, Marrero s. n. E Scholz (LPA, P); 8 Jul 2000, Marrero s. n. E Scholz (LPA); Pico de la Zarza, andén debajo del paloblanco, $28^{\circ} 6^{\prime} \mathrm{N}, 14^{\circ} 21^{\prime} \mathrm{W}, 8$ Apr 2000 , Marrero s. n. \& Scholz (LPA); Pico de La Zarza, $28^{\circ} 6^{\prime}$ N , $14^{\circ} 21^{\prime}$ W, 17 Jul 1987, Montelongo s. n. (LPA); Pico del Fraile, $28^{\circ} 5^{\prime} \mathrm{N}, 14^{\circ} 23^{\prime} \mathrm{W}$, Aug 2003, Scholz s. n. (LPA); Pico de la Zarza, El Esquinazo, $28^{\circ} 6^{\prime} \mathrm{N}, 14^{\circ} 21^{\prime} \mathrm{W}$, Apr 2009, Scholz s. $n$. (LPA); Pico del Fraile, $28^{\circ} 5^{\prime} \mathrm{N}, 14^{\circ} 23^{\prime} \mathrm{W}, 25$ Apr 1999, Scholz s. $n$. $\mathcal{E}$ Almeida (LPA)

Notes-This species has been included in this section provisionally and with hesitation, because only two specimens with distichous leaves in young shoots and culms have been observed; besides, Trisetum tamonanteae has unequal to subequal glumes, another typical character of this section. Marrero Rodríguez and Scholz (2013) separated this species from T. argenteum, T. distichophyllum, T. macrotrichum, and T. velutinum because these species present a glaucous-velutinous

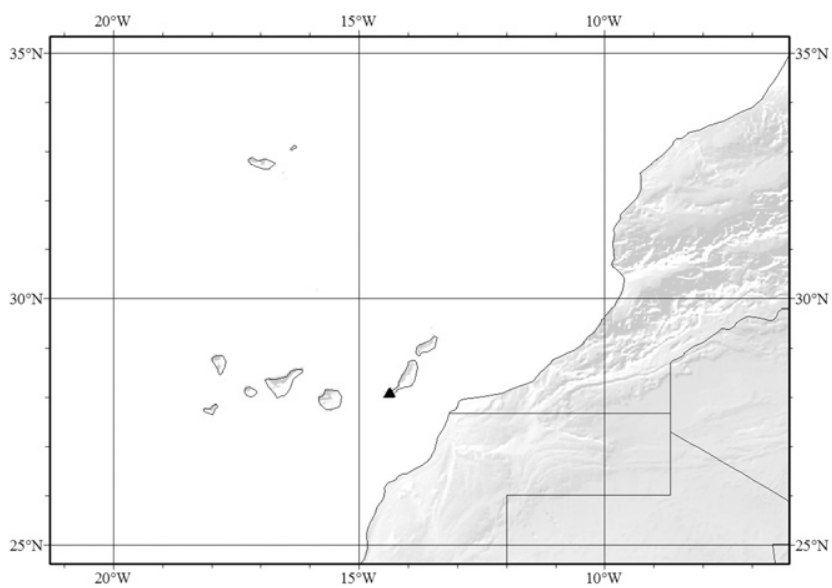

FIG. 12. Distribution of Trisetum tamonanteae.

hairiness and all basal and top leaf blades are clearly distichous. This is not the case in T. tamonanteae.

Doubtful and excluded names-

Avena distichophylla Host. The IPNI web attributed to Host (1802) the name Avena distichophylla. Host (1802), however, when he recorded Avena distichophylla, repeated the polynomial of Villars and indicated "Vill. delph. 2. p. 144. t. 4", suggesting that he was not publishing a nom. nov. Consequently the name "Avena distichophylla Host" should be forgotten. Avena distichophylla is also attributed to Schrader by some authors [i.e. Roemer and Schultes 2: 666 (1817)]. This case is similar to the one mentioned previously, since Schrader in his Flora Germanica 1: 380 (1806) is only indicating Villars' name, not describing a new species. Therefore, this name should be removed.

Avena distichophylla var. genuina Shuttlew., Mag. Zoo. Bot. 2: 17. 1838, nom. inval. (art. 24.3)

Avena penicillata Willd. ex Steud., Nomencl. Bot., ed. 2, 1: 172. 1840, nom. nud., pro syn.

Avena rotae De Not. ex Parl., Fl. Ital. 1: 264. 1850, nom. nud., pro syn.

Trisetum carpaticum (Host) Roem. \& Schult., Syst. Veg. 2: 663 (1817). This forgotten name is based on Avena carpatica Host, Icon. Descr. Gram. Austriac. 4: 18, Table 31. 1809 which, according to the Host's drawing, belongs to Avenula pubescens (Huds.) Dumort.

Trisetum gmelinii ["Gmelini"] Trin., Mém. Acad. Imp. Sci. Saint-Pétersbourg, Sér. 6, Sci. Math., Seconde Pt. Sci. Nat. 4(2): 15. Mar 1836, nom. inval. Bromus cinereus S.G. Gmel. ex Trin., Mém. Acad. Imp. Sci. Saint-Pétersbourg, Sér. 6, Sci. Math., Seconde Pt. Sci. Nat. 4: 15. Mar 1836, pro syn. Avena gmelini (Trin.) Nyman, Suppl. Syll. Fl. Eur.: 71. 1865. TYPE: SPAIN. in collibus siccis argillaico-sabulosis madriti, ex hb. Schreb. (lectotype, here designated, LE-TRIN-1893.1!). This specimen was send to Trinius by Schreber and there is no collector indicated on the label. It correspond to Trisetaria scabriuscula (Lag.) Paunero, a common species around Madrid.

Trisetum rigidum f. minor Kotschy, nom. nud., in sched. (P2255865!).

Trisetum rigidum f. colorata Bornm., nom. nud., in sched. (B!). 
Trisetum rigidum f. major Bornm., nom. nud., in sched. (BM1134945!).

Trisetum rigidum f. vivipara T. Alex., nom. nud., in sched. (LE!).

ACKNOWLEDGMENTS. This work was financed by Flora iberica CGL2014-52787-C3-1-P, CGL2012-32914 and CGL2015-66161-P, both projects of the Spanish Government. We are indebted to the curators and staff of the herbaria mentioned in Material and Methods for kindly providing specimens. We thank the editor and anonymous reviewers for their critical reviews of the manuscript and for their helpful suggestions during the editing process. We thank A. Quintanar for his revision of an earlier version of this manuscript. Ramón Morales for translations of manuscripts in German. Marta Fernández-Albert and Toni Buira for their technical support. Anne Martin and Maria Morck for their help with the English revision. Robert Vogt, Barina Zoltán, Francesco Zonca, Zbigniew Mirek, Manana Khutsishvili, and Mats Hjertson helped in finding rare literature, type materials, and geographical localities. N. N. Tzvelev, Dmitry Geltman, Roman Ufimov, Elena Glazkova, Denis Melnikov, and the rest of the personnel from the Komarov Institute for their help in many aspects and their welcoming attitude during our stay.

\section{Literature Cited}

Ascherson, P. F. A. and K. O. R. P. P. Graebner. 1899. Synopsis der Mitteleuropäischen Flora vol. 2. Leipzig: Wilhelm Engelmann.

Baumgarten, J. C. G. 1817. Enumeratio Stirpium magno Transsilvaniae vol. 3. Wien: Libraria Comesina.

Beauverd, G. 1934. Résultats de l'Herborisation de 1933 dans le Bassin de la Haute Durance (Dauphiné). Bulletin de la Societé Botanique de Genève, sér. 2 26: 122.

Benedí, C. and Ll. Sáez. 1996. Propósitos y despropósitos de Reineck y sus prosélitos. Anales del Jardin Botanico de Madrid 54: 570-574.

Beuret, E. 1974. Cytologie et distribution de Trisetum distichophyllum (Vill.) P.B. et Trisetum argenteum (Willd.) Roem. et Schult. Bull. Soc. Neuchâteloise. Science and Nature 97: 235-247.

Boissier, P. E. 1846. Diagnoses Plantarum Orientalium Novarum sér. 1, Nº 7 . Leipzig: Apud B. Hermann.

Boissier, P. E. 1854. Diagnoses Plantarum Orientalium Novarum sér. 2, № 13. Como: Typis Henrici Wolfrath.

Bolòs, O. and J. Vigo. 2001. Flora dels Països Catalans, vol. IV. Monocotiledònies. Barcelona: Editorial Barcino.

Burdet, H. M., A. Charpin, and F. Jacquemoud. 1981. Types nomenclaturaux des taxa ibériques décrits par Boissier ou Reuter. I. Gymnospermes à Graminées. Candollea (Genève) 36: 543-584.

Chrtek, J. 1965. Bemerkungen zur Gliederung der Gattung Trisetum Pers. Botaniska Notiser 118: 210-224.

Chrtek, J. 1967. Trisetum rechingeri, eine neue art der Flora Griechelands. Acta Universitatis Carolinae. Biologica 1966: 91-94.

Chrtek, J. 1968. Bemerkungen zu einigen Asien-Taxa der gattung Trisetum. Acta Universitatis Carolinae. Biologica 1967: 95-102.

Clayton, W. D. and S. A. Renvoize. 1986. Genera Graminum, grasses of the world. Kew Bulletin. Additional series 13: 1-389.

Devesa, J. A. and C. Romero Zarco. 1984. Numeros cromosomáticos para la flora española. 331-337. Lagascalia 12: 290-292.

Dumortier, B. C. 1824. Observations sur les Graminées de la Flore Belgique. Tournay: Impr. de J. Casterman, Ainé.

Du Riezt, G. E. 1930. The fundamental units of Biological Taxonomy. Svensk Botanisk Tidskrift 24: 333-428.

Edgar, E. 1998. Trisetum Pers. (Gramineae: Aveneae) in New Zealand. New Zealand Journal of Botany 36: 539-564.

Ellis, R. P. 1976. A procedure for standardizing comparative leaf anatomy in the Poaceae I. The leaf blade as viewed in transverse section. Bothalia 12: 65-109.

Ellis, R. P. 1979. A procedure for standardizing comparative leaf anatomy in the Poaceae II. The epidermis as seen in surface view. Bothalia 12: 641-671.

ESRI 2008. ArcGIS v. 9.3. Redlands, CA: Environmental Systems Research Institute.

Finot, V. L., P. M. Peterson, R. J. Soreng, and F. Zuloaga. 2004. A revision of Trisetum, Peyritschia, and Sphenopholis (Poaceae: Pooideae: Aveninae) in Mexico and Central America. Annals of the Missouri Botanical Garden 91: 1-30.

Finot, V. L., P. M. Peterson, R. J. Soreng, and F. O. Zuloaga. 2005a. A Revision of Trisetum and Graphephorum (Poaceae: Pooideae: Aveninae) in North America, North of Mexico. Sida 21: 1419-1453.
Finot, V. L., P. M. Peterson, F. O. Zuloaga, R. J. Soreng, and O. Matthei. 2005b. A Revision of Trisetum (Poaceae: Pooideae: Aveninae) in South America. Annals of the Missouri Botanical Garden 92: 533-568.

Finot, V. L., C. M. Baeza, and O. Matthei. 2006. Micromorfología de la epidermis de la lemma de Trisetum y géneros afines (Poaceae, Pooideae). Darwiniana 44: 32-57.

Font Quer, P. 1946. Sertulum Cavanillesianum enneanthum. Anales del Jardin Botanico de Madrid 6: 487-495.

Forsskål, P. 1775. Flora aegyptiaco-arabica, ed. C. Niebuhr. Copenhague: Hauniae: ex officina Mölleri.

Frey, L. 1991. New chromosome numbers in Trisetum (Poaceae). Fragmenta Floristica et Geobotanica 35: 97-99.

GPWG (Grass Phylogeny Working Group). 2001. Phylogeny and subfamilial classification of the grasses (Poaceae). Annals of the Missouri Botanical Garden 88: 373-457.

Hayek, A. 1932. Prodromus florae peninsulae Balcanicae. Repertorium Specierum Novarum Regni Vegetabilis 30: (Beiheft): 1-472.

Hermann, F. 1956. Flora von Nord- und Mitteleuropa. Stuttgart: Gustav Fischer Verlag.

Host, N. T. 1802. Icones et Descriptiones Graminum Austriacorum 2: 39.

Hultén, O. E. G. 1959. The Trisetum spicatum complex. Svensk Botanisk Tidskrift 53: 203-228.

Jávorka, S. 1919. Trisetum albanicum Jáv. spec. nova. Magyar Botanikai Lapok 18: $1-2$.

Jonsell, B. 1980. Trisetum Pers. Pp. 220-224 in Flora Europaea vol. 5, eds. T. G. Tutin, V. H. Heywood, N. A. Burges, D. M. Moore, D. H. Valentine, S. M. Walters, and D. A. Webb. Cambridge, U. K.: Cambridge University Press.

Kharadze, A. L. 1938. Trisetum teberdense (Litv.) A. Kharadze. Zametki po Sistematike i Georgrafii Rasteni (Tbilisi) 1: 7-23.

Koch, S. D. 1979. The relationship of three Mexican Aveneae and some new characters for distinguishing Deschampsia and Trisetum (Gramineae). Taxon 28: 225-235.

Kováts, D. 2000. Plant types of Sándor Jávorka in the Hungarian Natural History Museum in Budapest III. Annales Historico-Naturales Musei Nationalis Hungarici 92: 21-40.

Lippert, W. 2006. Chromosomenzahlen von Pflanzen aus Bayern und anderen Gebieten. Berichte der Bayerischen Botanischen Gesellschaft 76: 85-110.

Maire, R. 1942. Contributions à l'étude de la Flore de l'Afrique du Nord. Bulletin de la Société d'Histoire Naturelle de l'Afrique du Nord 33: 88-102.

Marrero Rodríguez, A. and S. Scholz. 2013. Trisetum tamonanteae (Poaceae, Aveninae), a new species from Fuerteventura, Canary Islands, Spain. Willdenowia 43: 45-57.

Metcalfe, C. R. 1960. Anatomy of Monocotyledons. I. Gramineae. Oxford: Oxford University Press.

Mosulishvili, M. 1991. Conspectus systematis specierum Caucasicarum subribus Koeleriinae Aschers. et Graebn. (Gramineae). Zametki po Sistematike i Geografii Rastenii 42: 32-45.

Nicora, E. G. 1978. Gramineae. Pp. 1-579 in Flora Patagónica 3, ed. M. N. Correa. Buenos Aires: Colección Científica del INTA.

Paunero, E. 1950. Las espécies españolas del género Trisetaria Forsk. Anales del Jardin Botanico de Madrid 9: 501-582.

Persoon, C. H. 1805. Synopsis Plantarum vol. 1. Paris: Apud. Carol. Frid. Cramerum.

Pfeiffer, L. G. K. 1871-1873. Nomenclator botanicus vol. 1. Kassel: Cassellis: Sumptibus Theodori Fischeri.

Pierce, S., C. M. Stirling, and R. Baxter. 2003. Pseudoviviparous reproduction of Poa alpina var. vivipara L. (Poaceae) during long-term exposure to elevated atmospheric $\mathrm{CO}_{2}$. Annals of Botany 91: 613-622.

Pignatti, S. 1982. Flora d'Italia vol. 3. Bologne: Edagricole.

Poiret, J. L. M. 1808. Encyclopédie Méthodique, Botanique vol. 8. Paris: Panckoucke.

Quintanar, A. and S. Castroviejo. 2010. Proposal to conserve Trisetum against Trisetaria (Gramineae). Taxon 59: 1602-1603.

Quintanar, A. and S. Castroviejo. 2013. Taxonomic Revision of Koeleria (Poaceae) in the Western Mediterranean Basin and Macaronesia. Systematic Botany 38: 1029-1061.

Quintanar, A., S. Castroviejo, and P. Catalán. 2007. Phylogeny of tribe Aveneae (Pooideae, Poaceae) inferred from plastid trnT-F and nuclear ITS sequences. American Journal of Botany 94: 1554-1569.

Quintanar, A., P. Catalán, and S. Castroviejo. 2010. A review of the systematics and phylogenetics of the Koeleriinae (Poaceae: Poeae). Diversity, phylogeny, and evolution in the Monocotyledons. Denmark: Aarhus University Press.

Randall, J. L. and K. W. Hilu. 1986. Biosistematic studies of North American Trisetum spicatum (Poaceae). Systematic Botany 11: 567-578.

Rechinger, K. H. 1970. Flora des Iranischen Hochlandes und der Umrahmenden Gebirge: Persien, Afghanistan, Teile Von West-Pakistan, Nord Iraq, 
Azerbaidjan, Turkmenistan, Gramineae vol. 70. Graz: Akademische Druck.

Roemer, J. J. and J. A. Schultes. 1817. Systema vegetabilium vol. 2. Stuttgard: J.G. Cottae.

Rohlena, J. 1942. Conspectus Florae Montenegrinae. Praha: Societatis botanicae bohemicae.

Rothmaler, W. 1935. Generum plantarum ibericarum revisio critica III Euphrasia L. Cavanillesia 7: 5-28.

Sãvulescu, T. 1972. Flora Reipublicae Socialisticae Romania vol. XII. Bucarest: Academia Republicii Socialiste România.

Schrader, H.A. 1806. Flora Germanica vol. 1. Göttingen: apud Henricum Dieterich.

Schultes, J.A. and J.H. Schultes. 1827. Mantissa vol. 3. Stuttgard: J.G. Cottae

Soreng, R. J., P. M. Peterson, G. Davidse, E. J. Judziewicz, F. O. Zuloaga, T. S. Filgueiras, and O. Morrone. 2003. Catalogue of the New World grasses (Poaceae): IV. Subfamily Pooideae. Contributions from the United States National Herbarium 48: 1-730.

Soreng, R. J., J. I. Davis, and M. A. Voionmaa. 2007. A phylogenetic analysis of Poaceae tribe Poeae sensu lato based on morphological characters and sequence data from three plastid-encoded genes: Evidence of reticulation, and new classification for the tribe. Kew Bulletin 62: 425-454.

Soreng, R. J., P. M. Peterson, K. Romaschenko, G. Davidse, F. O. Zuloaga, E. J. Judziewicz, T. S. Filgueiras, J. I. Davis, and O. Morrone. 2015. A worldwide phylogenetic classification of the Poaceae (Gramineae). Journal of Systematics and Evolution 53: 117-137.

Stuessy, T. F., D. J. Crawford, D. E. Soltis, and P. Soltis. 2014. Plant Systematics. The origin, interpretation, and ordering of plant biodiversity. Königstein: Koeltz Scientific Books.

Thiers, B. 2017. Index Herbariorum: A global directory of public herbaria and associated staff. New York Botanical Garden's Virtual Herbarium. http://sweetgum.nybg.org/science/ih/ (last accessed: January 2017).

Tolivia, D. and J. Tolivia. 1987. Fasga: A new polychromatic method for simultaneous and differential staining of plant tissues. Journal of Microscopy 148: 113-117.

Tzvelev, N. N. 1971. [1970]. De generibus Trisetum Pers. et Koeleria Pers. in URSS notulae systematicae. Novosti Sistematiki Vysshikh Rastenii 7: $59-73$.

Tzvelev, N. N. 1976. Zlaki SSSR. Saint Petersburg: Nauka Publishers [English translation: Grasses of the Soviet Union. 1983. New Delhi: Amerid Publishing.]

Tzvelev, N. N. 1989. The system of grasses and their evolution. Botanical Review 55: 141-204.

Watson, L. and M. J. Dallwitz. 1992. The grass genera of the world. Wallingford: C. A. B. International.

APPENDIX 1. Index to numbered collections cited. The numbers in parentheses refer to the corresponding species in the text. Mixed collections are indicated by a slash (e.g., 1/2).

Abdaladze, O. et al. 332 (4.a), 409 (4.a); Aedo, C. 18599 (6), 11798 (4.a); Aellen, P. 1898 (4.a), 1982 (4.a); Alexeenko, Th. 1101 (4.a), 392 (4.a), 5623 (4.a), 6104 (4.a), 7396 (4.a), 9071 (4.a); Barberá, P. et al. 941PB (6), 942PB (6); Bobrov, A.E. et N.N. Tzvelev 574 (4.a); Boissier, E. 187 (6); Bornmüller, J. 33 (4.a); Bornmüller, J. et A. Bornmüller 8381 (4.a), 8382 (4.a), 8383 (4.a), 8384 (4.a), 8385 (4.a), 8385 b (4.a); Borodin, I. 197 (4.a); Bot. Exk. 447 (1), 740 (1); Bourgeau, E. 1519 (6); Castroviejo, S. et al. 11213 SC (1); Charpin, A. 15786 (2), 19140 (1);
Charpin, A. et R. Salanar AC17607 (1); Clarke, C.B. 48718 (1); Danser, B.H. 5050 (1); Del Campo, P. 93 (6), 98 (6); Dersch, G. 4488 (2); Dini-Arazm 12132 (4.a); Dzens-Litovskaya, N. 99 (4.a); Eckardt, T. 615 (1), 1056 (1); Egeröd, K. 9034 (1); Egorova, T.V. et al. 433 (4.a), 1477 (4.a); Fernández Casas, F.J. 963 B (6); Furse, P. 3141 (4.a); Gagnidze, R. et al. 2524 (4.a), 2941 (4.a); Gauba, D.E. 1243 (4.a); Gladkov, I. 22 (5.a); Grossheim, A. 76 (4.a); Herrero, A. 2718 (4.a); Holmberg, O.R. 974 (4.a); Holtz, F. et al. 978 (4.a); Hörandl, E. 1739 (1); Huter, R. 1171 (2); Huter, R. et al. 1171 (6); Jacobs, J. 3893 (1); Kalheber, H. 96-1385 (2); Kolenati, F.A. 2204 (4.a); Kotschy, TH. 391 (4.a), 393 (4.a); Kotseruba, V.V. 30 (4.a), 56 (5.b); Lachashvili, N. 189 (4.a); Lippert, W. 26158 (2); Litwinow, D.I. 257 (4.a), 2809 (4.a); Litzler, P. 77/765E (6); López, G. 890 GF (6); Lütkemüller, J. 2253 (1); Merxmüller, H. et W. Wiedmann 5533 (2), 5534 (2); Meyer, C.A. 60 (5.a); Milne-Redhead, E. 2316 (1); Moutin, R. et J. Bernard 475 (1); Müller, J. 10983 (2); Nakhutsrishvili, G. et O. Abdaladze 103 (4.a); Novopokrovskiy, I.V. 681 (4.a); Podlech, D. 42147 (1); Poretskiy, A. et G. Shults 543 (4.a); Porta, P. et G. Rigo 648 (6); Prokhanov, Y.I. 339 (4.a); Prokhanov, Y.I. et N.T. Cheldyshev 163 (4.a); Raus, T. 4431 (4.a); Rechinger, K.H. 40731 (4.a), 48369 (4.a), 57178 (4.a), 5891 (4.a), 6091 (4.a); Rechinger, K.H. et F. Rechinger 6447 (4.a), 6509 (4.a); Reverchon, E. et A. Derbez 255 (1); Riera, J. 3135 (6); Riera, J. 3151 (6), 3719 (6), 3729 (6); Riera, J. et J.V. Andrés JRV-4480 (6); Rigo, G. 1411 (2); Romero, C. C170/81 (6); Schiffers, E.V. et T.A. Moreva 5 (4.a); Soler, J.X. et M. Signes 7265 JXS (6), JXS-7339 (6); Sommier, S. et E. Levier 1359 (4.a/5.a/5.b); Soreng, R.J. 8009 (4.b); Soriano, P. 0917PS (6), 1652PS (6); Stebler, F.G. et C.J. Schröter 114 (1); Tatli, A. 4865 (4.a); Tzvelev, N.N. et S. Cherepanov 160 (4.a); Vasilchenko, I.T. et al. 668 (4.a); Wagenitz, G. 1738 (1/2); Wagenitz, G. et F. Hellwig 4707 (6); Wendelbo, P. 1218 (4.a); Woronow, G. 6256 (4.a); Zernov, A.S. 6527 (4.b), 6934 (5.a), 7263 (5.a); Zündorf, H.J. 24247 (5.b); Zündorf, H.J. et A. Gerth 25658 (5.b).

APPENDIX 2. List of the quantitative characters measured.

Plant height $(\mathrm{cm})$; Culm diameter $(\mathrm{mm})$; Culm indumentum length $(\mathrm{mm})$; Number of nodes; Basal leaf sheath indumentum length ( $\mathrm{mm})$; Basal leaf blade length $(\mathrm{cm})$; Basal leaf blade width $(\mathrm{mm})$; Basal leaf blade indumentum length (mm); Top culm leaf sheath length (cm); Top culm leaf blade length $(\mathrm{cm})$; Top culm leaf blade width $(\mathrm{mm})$; Top culm leaf blade indumentum length ( $\mathrm{mm})$; Inner collar region indumentum length $(\mathrm{mm})$; Ligule length $(\mathrm{mm})$; Ligule indumentum length $(\mathrm{mm})$; Basal node of the panicle indumentum length $(\mathrm{mm})$; Panicle length $(\mathrm{cm})$; Panicle width $(\mathrm{cm})$; Rachis indumentum length $(\mathrm{mm})$; Longest basal branches length $(\mathrm{cm})$; Pedicel length $(\mathrm{mm})$; Pedicel indumentum length $(\mathrm{mm})$; Spikelet length $(\mathrm{mm})$; Spikelet width (mm); Number of flowers per spikelet; Ratio lower glume length/upper glume length; Lower glume length (mm); Lower glume width (mm); Ratio lower glume width/lower glume length; Lower glume indumentum length (mm); Number of lower glume nerves; Upper glume length (mm); Upper glume width (mm); Ratio upper glume width/ upper glume length; Upper glume indumentum length (mm); Number of upper glume nerves; Rachilla segments between first and second floret length $(\mathrm{mm})$; Rachilla segments between first and second floret indumentum length $(\mathrm{mm})$; Lemma length (mm); Lemma width (mm); Ratio lemma width/lemma length; Lemma indumentum length $(\mathrm{mm})$; Apical teeth length $(\mathrm{mm})$; Aristule length (mm); Awn length (mm); Awn insertion from the base (mm); Awn insertion from the base length/lemma length; Awn indumentum length $(\mathrm{mm})$; Callus length $(\mathrm{mm})$; Callus indumentum length $(\mathrm{mm})$; Palea length $(\mathrm{mm})$; Palea width $(\mathrm{mm})$; Ratio palea length/lemma length; Palea teeth length (mm); Lodicule length ( $\mathrm{mm})$; Anther length ( $\mathrm{mm})$; Ovary length ( $\mathrm{mm}$ ); Caryopsis length $(\mathrm{mm})$; Caryopsis width (mm). 